ACS Chem Neurosci. 2016 October 19; 7(10): 1418-1432. doi:10.1021/acschemneuro.6b00182.

\title{
Effect of Substitution on the Aniline Moiety of the GPR88 Agonist 2-PCCA: Synthesis, Structure-Activity Relationships, and Molecular Modeling Studies
}

\author{
Chunyang Jin*, Ann M. Decker, Danni L. Harris, and Bruce E. Blough \\ Center for Drug Discovery, Research Triangle Institute, Research Triangle Park, North Carolina \\ 27709, United States
}

\begin{abstract}
GPR88, an orphan receptor richly expressed in the striatum, is implicated in a number of basal ganglia-associated disorders. In order to elucidate the functions of GPR88, an in vivo probe appropriate for CNS investigation is required. We previously reported that 2-PCCA was able to modulate GPR88-mediated cAMP production through a $\mathrm{G} a_{\mathrm{i}}$-coupled pathway. Early structureactivity relationship (SAR) studies suggested that the aniline moiety of 2-PCCA is a suitable site for diverse modifications. Aimed at elucidating structural requirements in this region, we have designed and synthesized a series of analogues bearing a variety of substituents at the phenyl ring of the aniline moiety. Several compounds (e.g., 5j, 5o) showed improved or comparable potency, but have lower lipophilicity than 2-PCCA (clogP 6.19). These compounds provide the basis for further optimization to probe GPR88 in vivo functions. Computational studies confirmed the SAR trends and supported the notion that $4^{\prime}$-substituents on the biphenyl ring exit through a largely hydrophobic binding site to the extracellular loop.
\end{abstract}

\section{Graphical Abstract}
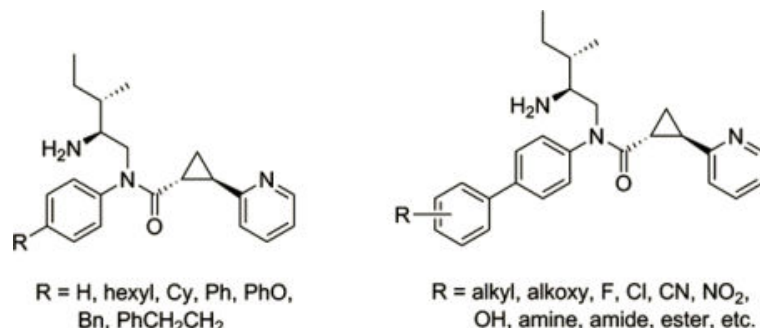

$\mathrm{R}=$ alkyl, alkoxy, $\mathrm{F}, \mathrm{Cl}, \mathrm{CN}, \mathrm{NO}_{2}$, $\mathrm{Bn}, \mathrm{PhCH}_{2} \mathrm{CH}_{2}$ $\mathrm{OH}$, amine, amide, ester, etc.

\footnotetext{
*Corresponding Author: Chunyang Jin, Research Triangle Institute, Post Office Box 12194, Research Triangle Park, NC 27709. Telephone: 919 541-6328. Fax: 919 541-8868. cjin@rti.org.

Supporting Information

The Supporting Information is available free of charge on the ACS Publications website at DOI: 10.1021/acschemneuro.6b00182. Copies of HPLC results of 4a-g and 5a-aa, QSAR models A and B description, sequence alignment of GPR88 with selected GPCRs and $\beta 1$-adrenergic receptor templates, PDB coordinates for GPR88 homology model (PDF)

Author Contributions

C.J. designed the studies, performed the synthesis and analyzed the data, A.M.D. performed the biological assays and analyzed the biological data, D.L.H. performed computational studies, and B.E.B. analyzed the biological data. All authors wrote the manuscript.

Notes

The authors declare no competing financial interest.
} 


\section{Keywords}

Orphan GPR88; 2-PCCA; SAR; molecular modeling

The G protein-coupled receptors (GPCRs) constitute the largest family of membrane proteins and mediate most cellular responses to hormones and neurotransmitters. GPCRs also play important roles in disease pathogenesis and account for more than $30 \%$ of targets of marketed drugs. ${ }^{1}$ Analysis of the human genome revealed more than 800 GPCR sequences, of which around 140 GPCRs, excluding olfactory receptors, are still classified as orphan receptors for which endogenous ligands are unknown. ${ }^{2}$ The complex biology and potential for drug therapy within this class provide strong incentives for small molecule probe development to enable modulation of individual receptors and facilitate elucidation of their biological functions. ${ }^{3}$

GPR88 is an orphan receptor highly expressed in the CNS, with particularly robust expression in the striatum throughout the dorsal and ventral areas. ${ }^{4}$ Transcriptional profiling studies have revealed $G p r 88$ gene expression is altered by conditions related to Parkinson's disease, ${ }^{4 \mathrm{c}}$ schizophrenia, ${ }^{5}$ bipolar disorder,${ }^{6}$ depression, ${ }^{7}$ and drug addiction. ${ }^{8}$ Interestingly, both receptor up- and down-regulation have been observed depending on the treatment and brain region. For example, in dopamine-depleted striatum, Gpr88 expression was differentially regulated in striatonigral and striatopallidal medium spinal neurons (MSNs), and L-DOPA treatment normalized Gpr 88 expression levels. ${ }^{4 c}$ Gpr 88 gene expression was up-regulated in the prefrontal cortex following methamphetamine exposure ${ }^{6 \mathrm{~b}}$ and downregulated in the amygdala following treatment with the NMDA receptor antagonist MK-801. 5

More direct evidence of GPR88 function was obtained from GPR88 knockout studies. GPR88 knockout mice demonstrated disrupted prepulse inhibition of startle response, a phenotype of schizophrenia, and exhibited $\mathrm{D}_{2}$ receptor hypersensitivity, which were normalized to control levels by antipsychotic drug administration. ${ }^{9}$ In another study, the GPR88 knockout animals exhibited increased locomotion, poor motor coordination and impaired cue-based learning. ${ }^{10}$ Examination of MSNs electrophysiology in brain slices revealed decreased tonic GABAergic inhibition and increased glutamatergic excitation, which promoted enhanced firing rates observed in vivo. ${ }^{10}$ In addition, GPR88 re-expression normalized these impaired behaviors and electrophysiological properties, indicating that GPR88 dysfunction may contribute to abnormal behaviors observed in basal gangliaassociated disorders such as Parkinson's disease and schizophrenia. Interestingly, a recent study showed that the GPR88-deficient mice performed better in spatial tasks and reduced levels of anxiety, indicating GPR88 may also play a role in cognitive and anxiety disorders. ${ }^{11}$

Despite emerging pharmacological implications of GPR88, the signaling mechanisms and biological functions of this orphan receptor are still largely unknown due to the lack of potent and selective native or synthetic ligands. We previously reported that 2-PCCA [( $1 R^{*}$, $\left.2 R^{*}\right)$-2-(pyridin-2-yl)-cyclopropanecarboxylic acid ((2S,3S)-2-amino-3-methylpentyl)-( $4^{\prime}$ propylbiphenyl-4-yl)-amide (1); Figure 1] was able to modulate GPR88-mediated cAMP 
production through a $\mathrm{G} a_{\mathrm{i}}$ - coupled pathway. ${ }^{12}$ Unfortunately, 2-PCCA has a high calculated lipophilicity ( $\log \mathrm{P} 6.19)$ and was reported to be a P-glycoprotein substrate, which might limit its effectiveness as a tool. ${ }^{13}$ In order to elucidate the functions of GPR88, an improved in vivo agonist probe appropriate for CNS investigation is required. Early structure-activity relationship (SAR) studies suggest that the aniline moiety of 2-PCCA is a suitable site for diverse modifications. ${ }^{12,13}$ Aimed at elucidating SAR requirements in this region, we have designed and synthesized a series of analogues (4a-g, Figure 1 and $\mathbf{5 a - a a}$, Figure 2) bearing a variety of substituents at the phenyl ring of the aniline moiety. In this paper, we report the SAR and computational studies of these compounds with the goal of improving potency and lowering the lipophilicity.

\section{RESULTS AND DISCUSSION}

\section{Chemistry}

The overall approach to the synthesis followed methods detailed in our earlier work. ${ }^{12}$ Synthesis of the designed compounds $\mathbf{4 a - g}$ is outlined in Scheme 1. Reductive amination of aldehyde 7, prepared by Dess-Martin oxidation of commercially available (-)-(2S,3S)- $N$ Boc-2-amino-3-methyl-1-pentanol, with 4-substituted aniline $\mathbf{6 a - h}$ afforded amine $\mathbf{8 a - h}$ in $46-77 \%$ yields. Amide formation with the acid chloride of racemic ( \pm -trans-2-(pyridin-2yl)-cyclopropanecarboxylic acid gave 9a-h in 32-72\% yields. Removal of the Boc protecting group of $\mathbf{9 b}-\mathbf{h}$ with $4 \mathrm{M} \mathrm{HCl}$ in dioxane provided target compounds $\mathbf{4 a -} \mathbf{a}$ in 8397\% yields.

Synthesis of the biphenyl derivatives 5a-aa is illustrated in Scheme 2. Suzuki coupling of the bromo compound 9a with an appropriate arylboronic acid under microwave conditions gave intermediates 10a-aa in the range of 51-81\% yields. Deprotection of the Boc group furnished 5a-aa in $86-100 \%$ yields. Target compounds $\mathbf{4 a -}$ a and $\mathbf{5 a - a a}$ were determined to be $1: 1$ mixture of $(1 R, 2 R)$ - and $(1 S, 2 S)$-enantiomers, differentiating at the configuration of the trans-substituted cyclopropane ring, by ${ }^{1} \mathrm{H}$ NMR and HPLC analyses.

\section{Biological Results and SAR Studies}

We previously demonstrated that 2-PCCA (1, Figure 1) inhibited isoproterenol-stimulated cAMP accumulation with $\mathrm{EC}_{50}=911 \mathrm{nM}$ in HEK293 cells stably expressing the human GPR88 receptor and the GloSensor-22F cAMP construct, indicating that GPR88 is coupled to the $\mathrm{G} a_{\mathrm{i}}$ subunits. ${ }^{12}$ Recently, $\mathrm{Bi}$ et al. reported that the $(1 R, 2 R)$-enantiomer of 2-PCCA exhibited an $\mathrm{EC}_{50}$ of $3.1 \mathrm{nM}$ in a HTRF (Cisbio Bioassays) cAMP assay. ${ }^{13}$ The discrepancy between the $\mathrm{EC}_{50}$ values of 2-PCCA is possibly due to the sensitivity of the two different assay systems. In order to facilitate the SAR study and discovery of potent GPR88 ligands, a reliable and sensitive assay is needed. Thus, we created a Chinese Hamster Ovary (CHO) cell line stably expressing the PPLS-HA-GPR88 construct and measured cAMP levels using the Lance assay platform (PerkinElmer). A preprolactin leader sequence (PPLS) and an influenza hemagglutinin (HA) tag were used in the construct to facilitate membrane expression and immunostaining of GPR88 in CHO cells. In the stable PPLS-HA-GPR88 CHO cells, 2-PCCA and its pure enantiomer 2 had $\mathrm{EC}_{50}$ values of 116 and $56 \mathrm{nM}$, respectively, shown in Figure 1. The phenyl analogue 3 possessed an $\mathrm{EC}_{50}$ of $233 \mathrm{nM}$. 
Early SAR studies suggest that the aniline moiety of 2-PCCA is a suitable site for optimization. ${ }^{12,13}$ It appears that the phenyl ring of the aniline moiety can tolerate substitution at the 4-position, in some cases leading to improved potency. In order to gain additional information about the structural requirements in this region for obtaining high potency ligands with possibly improved drug-like properties (e.g., $\operatorname{cog} \mathrm{P}<5$ ), we have synthesized a series of analogues (4a-g, Figure 1 and $\mathbf{5 a}-\mathbf{a a}$, Figure 2$)$ and tested their agonist activity in the Lance cAMP assay. These compounds bear a variety of substituents at the phenyl ring of the aniline moiety to systematically evaluate the effects of size, polarity, lipophilicity, and steric and electronic tolerance. The unsubstituted analogue 4a was significantly less potent than 2-PCCA (2760 vs $116 \mathrm{nM}$, Figure 1). Addition of a hexyl group at the 4-position of the phenyl ring led to $4 \mathbf{b}$, which regained most of the potency $\left(\mathrm{EC}_{50}=421 \mathrm{nM}\right)$, indicating a hydrophobic pocket may be present in the binding site to interact with this region. Replacing the hexyl group with cyclohexyl $(\mathbf{4 c})$ or phenyl (4d) further improved the $\mathrm{EC}_{50}$ to $283 \mathrm{nM}$. However, moving the distal phenyl group in $\mathbf{4 d}$ away from the aniline phenyl ring by replacement with phenoxy $(\mathbf{4 e})$, benzyl $(\mathbf{4 f})$, or phenethyl $(\mathbf{4 g})$ resulted in deteriorated activity. These findings suggested that an aromatic stacking interaction between the biphenyl moiety and the receptor might contribute to the agonist activity of 2-PCCA.

Substituent effect of the distal phenyl ring of $\mathbf{4 d}$ was next examined and the results are summarized in Table 1. Consistent with the previous findings, ${ }^{12}$ the 4-position of the distal phenyl ring tolerated small to medium sized alkyl substitutions with the ethyl analogue $\mathbf{5 c}$ $\left(\mathrm{EC}_{50}=85 \mathrm{nM}\right)$ being the most potent compound and the bulk $t$-butyl $\mathbf{5 f}\left(\mathrm{EC}_{50}=515 \mathrm{nM}\right)$ being the least potent compound in the series. Interestingly, the large alkyl substitutions, such as hexyl (5g), cyclohexyl (5h) and phenyl (5i), were also well tolerated giving good to moderate $\left(\mathrm{EC}_{50}=136-289 \mathrm{nM}\right)$ activity. This provided further evidence to support the previous hypothesis ${ }^{13}$ that the $4^{\prime}$-substitution at the biphenyl group likely extends into a hydrophobic pocket.

Improvement of the potency of $\mathbf{4 d}$ was also observed by adding small alkoxy groups at the 4-position (5j-m). Attachment of an additional methoxy group at the 3-position of $\mathbf{5 j}$ led to the 3,4-dimethoxy analogue $\mathbf{5 n}$, resulting in approximately 10 -fold loss of activity (96 nM vs $917 \mathbf{n M}$ ). The loss in potency of $\mathbf{5 n}$ was probably due to the limited steric tolerance proximal to the distal phenyl ring, as evidenced in $\mathbf{5 f}$. Somewhat surprisingly, the 3,4methylenedioxy analogue 5o possessed good activity with an $\mathrm{EC}_{50}$ of $204 \mathrm{nM}$. The electronwithdrawing groups $\left(\mathbf{5 b}, \mathbf{5 p}\right.$-u) were well tolerated at the 4-postion, with $\mathrm{EC}_{50}$ values ranging from $187 \mathrm{nM}$ to $351 \mathrm{nM}$. However, substituents containing a hydrogen-bond donor $(\mathbf{5 v}$ and $\mathbf{5 w})$ led to a significant decrease in activity. The dimethylamino group (5x) had an improved potency compared to the amino group in $\mathbf{5 w}$ (294 vs $1120 \mathrm{nM}$ ), suggesting that both basicity and lipophilicity of the substituents at the 4-postion may have a marked influence on the activity. Finally, substitution at the 4-position with an amide group $(\mathbf{5 y}, \mathbf{5 z}$, and 5aa) gave the least activity in the series.

Calculated physiochemical properties such as lipophilicity (clogP), topological polar surface area (TPSA), and derived values such as $\log B \mathrm{~B}$ are useful indicators of a compound's 
potential to penetrate the brain. In general, CNS drugs have clogP in the range of $2-4,{ }^{14}$ TPSA less than $76 \AA^{2,15}$ and $\operatorname{logBB}$ greater than $-1 .{ }^{16}$ These molecular descriptors were calculated for 2-PCCA, as well as selected compounds $\mathbf{5 a}, \mathbf{5 c}, \mathbf{5 j}, \mathbf{5 1}, \mathbf{5 m}$, and $\mathbf{5 0}$ (Table 2). 2-PCCA has the highest clogP value of 6.19. All other calculated compounds have clogP greater than 4, among which $\mathbf{5 0}$ has the lowest $\log \mathrm{P}=4.41$. 2-PCCA and all of the analogues, except 5o, have TPSA less than $76 \AA^{2}$. Even $\mathbf{5 o}$ has a TPSA $=77.68 \AA^{2}$, just above the recommended threshold (a TPSA cutoff of $90 \AA^{2}$ has also been suggested for CNS drugs ${ }^{17}$ ). All compounds have $\log B \mathrm{~B}$ values greater than -1 , predicting potential brain penetration.

\section{QSAR Analysis}

In a complementary effort to assess the SAR, we computed a series of properties of the substituents and analyzed their importance in explaining the observed variations in $\ln E C_{50}$ values. We computed optimized geometries of the hydrogen capped substituted biphenyl fragments using MOPAC $7^{18}$ and then computed shape/steric (Sterimol parameters/ globularity), ${ }^{19}$ polar surface area (PSA), volume, solvent accessible area, total hydrophobicity (TotalHyd) and HOMO/LUMO frontier orbital energetics using these semiempirical results in addition to GRAPHA assessments of the varied substituted biphenyl fragments. ${ }^{20} \operatorname{LogP}$ and Molar refractivity and number of rotatable bonds were computed using OpenBabel 2.3.2. ${ }^{21}$ Isotropic polarizabilities of the substituents were computed using GAMESS-UK ${ }^{22}$ at 6-31G ${ }^{* *}$ B3LYP DFT optimized geometries.

The covariance matrix of all the descriptors was computed to facilitate exploring QSAR employing descriptors with reasonable "orthogonality" in variance. In this way we maximized the linear independence of the descriptors and sought to explain the $\ln \mathrm{EC}_{50}$ variations via the QSAR description with the smallest number of possible terms while probing multiple facets of each of the substitutions. While many models were explored in an attempt to find explanations for the ln $\mathrm{EC}_{50}$ 's in terms of all the computed metrics, we focused on a buildup of three models and examined at each stage the impact of an additional descriptor.

Table 3 reports the best (model C) of three successive models, while the Supporting Information contains the description and statistics for the other two models $\mathrm{A}$ and $\mathrm{B}$ upon which model $\mathrm{C}$ was based. In the model $\mathrm{C}, \ln \mathrm{EC}_{50} \sim-1.0 \mathrm{~L}+1.7$ TotalHyd $+0.04 \mathrm{~V} \_\mathrm{S}$ + 0.1 PSA - 0.9 $\log \mathrm{P}+1.1 \mathrm{~B} 4+8.0$. The significance of both the "metric" within the given QSAR model as well as the ANOVA computations allowed one to decide on the significance of any improvement in the multivariate-linear least-squares with model expansion. The model table provides the coefficients of the metrics in the model QSAR equation, the standard error (an error level of the model computed from the sums-of-squares of deviations from the theoretical fit and the number of cases), the $t$-value (the $t$-statistic providing an indication of whether the coefficient is different than zero), and $\operatorname{Pr}$ (the $p$-value for the hypothesis test for which the $t$-value is the test statistic). As shown in Table 3, the $p$-value was $<0.05$ and indicated significance. At a 95\% confidence limit, the model had significance in explaining the variations of $\ln \mathrm{EC}_{50}$ in terms of the physiochemical descriptors probed. The $R^{2}$ value illustrated reasonable correlations in the fit described by the theoretical QSAR 
equation to the observed $\ln \mathrm{EC}_{50}$ value, and this coefficient of determination showed that the variables employed provided a $68 \%$ explanation for the variations in $\ln \mathrm{EC}_{50}$. Figure 3 shows a plot of the predicted versus the experimental $\ln \mathrm{EC}_{50}$ values.

The model C contains six physiochemical descriptors. PSA, $\log$, and total hydrophobicity are common features used in the assessment of drug candidates, but the L- and B4-Sterimol (L and B4, respectively) and the vibrational thermochemical (V_S) descriptors are much less well-known. An example of the L- and B4-Sterimol metrics for two ligands $\mathbf{5 j}$ and $\mathbf{5 o}$ is shown in Figure 4. The L-Sterimol axis is the longest dimension spanning the molecular surface of the substituent. The B4-Sterimol descriptor is orthogonal to this L-dimension and provides a metric of a width of the substituent to the L-axis. Such orthogonal descriptor captures the width of the binding pocket orthogonal to the long axis of the substituent.

The vibrational parameter $\left(\mathrm{V}_{-} \mathrm{S}\right)$ is a descriptor that captures a dynamical aspect of the ligand and possibly indicates a propensity for cooperative binding. While differential binding to the orthosteric site of a GPCR is not likely to result in significant receptor structural changes near the orthosteric site, small changes in a ligand's architecture may result in differential stabilization of activated conformations of the GPCR. The effect of the local fluctuations in a portion of a ligand substituent may bias larger cooperative structural changes at the intracellular side to conformations constituting an "active state". ${ }^{23,24}$ Small changes in a ligand's dynamics may in this way alter ionic locks/loop and helical structure at the intracellular region that modifies interactions with GPCR partners (e.g., G proteins, $\beta$ arrestin). While such a rationale for V_S remains speculative, there was a plausible connection of the vibrational parameter to the $\ln \mathrm{EC}_{50}$ in our QSAR model.

\section{Docking Analysis of $(1 R, 2 R)-2-\mathrm{PCCA}$}

While the QSAR model compared reasonably with the qualitative facets of the SAR deductions at the aniline moiety, we sought to discover if a homology model, based on existing crystallographic templates, would give useful information about the receptor binding site characteristics for this region. The focus of this limited structural exploration was to ascertain whether the QSAR is consistent with structural principles/orthosteric ligand binding site based on the docking and MMGBSA (Molecular Mechanics Generalized Born Surface Area $)^{25}$ scoring analysis of $(1 R, 2 R)$-2-PCCA.

Sequence alignments of human GPR88 with selected GPCR templates of known structure were performed using a BLOSUM62 matrix. While no GPCR crystal structures with high or moderate sequence identity ( $>40 \%$ by our own experience) to GPR 88 have yet been solved, the $\beta 1$-adrenergic receptor (PDB: $5 \mathrm{~A} 8 \mathrm{E}$ ) has a $26 \%$ sequence identity to GPR 88 deduced from a pairwise alignment. This template also has one of the highest sequence identities in the alignment of 9 GPCRs with GPR88 ( $\sim 20 \%$, see the Supporting Information). The $\beta 1-$ adrenergic receptor template was used to construct an initial backbone model of GPR88 employing Sali's MODELER v9.11, ${ }^{26}$ refining the backbone model "energetically" using simulated annealing with topological constraints. We next employed SCWRL ${ }^{27}$ rotamer exploration to find nonclashing side chain orientations for nonconserved residues with conserved residues between the target and template employing conservation assessments shown in the alignment. Conserved residue side chain from the $\beta 1$-adrenergic receptor 
template were initially retained in the SCWRL rotamer exploration. This initial level model was then energy minimized using AMBER $12^{28}$ to provide a homology model for docking and free-energy scoring.

We docked $(1 R, 2 R)$-2-PCCA using both Autodock VINA ${ }^{29}$ with AMBER 12 MMGBSA rescoring as well as GLIDE-SP (Schrödinger) ${ }^{30}$ followed by Prime-MMGBSA rescoring. As shown in Figure 5, the $4^{\prime}$-propylbiphenyl group in $(1 R, 2 R)$-2-PCCA pointed out of the orthosteric site into the extracellular loop region. The residue character along the binding site region housing the biphenyl is quite hydrophobic. The initial GPR88 homology model appears to be consistent with the results of QSAR analysis in that the long-spatial axis, parametrized at the L-Sterimol, is an important facet as is the overall hydrophobicity. The model is not, at this stage, predictive of $\ln ^{\mathrm{EC}_{50}}$. Refinement of the model using pure $(R, R)$ diastereomers is currently under investigation.

\section{CONCLUSIONS}

In summary, we have designed and synthesized a series of 2-PCCA analogues bearing a variety of substituents at the phenyl ring of the aniline moiety to determine the SAR in this region. The target compounds were evaluated in a Lance cAMP assay using stable PPLSHA-GPR88 CHO cells. SAR studies suggested that there is a hydrophobic pocket in the GPR88 binding site interacting with the aniline region of 2-PCCA. In addition, the $4^{\prime}$ position of the biphenyl group was well tolerated with alkyl, alkoxy and electronwithdrawing groups, but less tolerated with the substituents having a hydrogen-bond donor. Several compounds had a slightly improved or comparable potency, but lower calculated lipophilicity than 2-PCCA. Combined with calculated TPSA and $\operatorname{logBB}$ values, compound $\mathbf{5 j}$ may provide the basis for further optimization to develop an in vivo probe for GPR88 functional studies.

Exploration of both a computational QSAR and an initial homology model and docking of $(1 R, 2 R)$-2-PCCA supported the SAR conclusions. The findings of statistically sound models including both L- and B4-Sterimol parameters in the QSAR were consistent with the positioning of the $4^{\prime}$-substituted biphenyl encased in a largely hydrophobic GPR88 homology model binding site.

\section{METHODS}

\section{Chemistry}

General Methods-Melting points were determined using a MEL-TEMP II capillary melting point apparatus and are uncorrected. Nuclear magnetic resonance $\left({ }^{1} \mathrm{H} N \mathrm{NR}\right.$ and ${ }^{13} \mathrm{C}$ NMR) spectra were obtained on a Bruker Avance DPX-300 MHz NMR spectrometer. Chemical shifts are reported in parts per million (ppm) with reference to internal solvent. ${ }^{13} \mathrm{C}$ NMR data of diastereomeric mixtures were not reported due to the complicity of the spectra. Mass spectra (MS) were run on a PerkinElmer Sciex API 150 EX mass spectrometer. HRMS spectra were run on a Waters Synapt G2 HDMS Q-TOF mass spectrometer, using electrospray ionization in positive ion mode. Analytical thin-layer chromatography (TLC) was carried out using EMD silica gel $60 \mathrm{~F}_{254}$ TLC plates. TLC 
visualization was achieved with a UV lamp or in an iodine chamber. Flash column chromatography was done on a CombiFlash Companion system using Isco prepacked silica gel columns. Unless otherwise stated, reagent-grade chemicals were obtained from commercial sources and were used without further purification. All moisture- and airsensitive reactions and reagent transfers were carried out under dry nitrogen. Synthesis and characterization of compounds 1-3, 5a-c, 5e, 5h, 5p, 5q, and $5 \mathbf{t}$ have been previously reported. ${ }^{12}$ All synthesized compounds were $₹ 95 \%$ pure as determined by HPLC analyses (see the Supporting Information).

tert-Butyl \{(2S,3S)-1-[(4-Bromophenyl)amino]-3-methylpentan-2-yl\}carbamate (8a)-To a solution of (-)-(2S,3S)-N-Boc-2-amino-3-methyl-1-pentanol (2.17 g, 10.0 $\mathrm{mmol})$ in water-saturated $\mathrm{CH}_{2} \mathrm{Cl}_{2}(10 \mathrm{~mL})$ at room temperature was added Dess-Martin reagent $(8.90 \mathrm{~g}, 21.0 \mathrm{mmol})$ and the reaction was stirred for $1 \mathrm{~h}$. Additional water-saturated $\mathrm{CH}_{2} \mathrm{Cl}_{2}(5 \mathrm{~mL})$ was added every $15 \mathrm{~min}$ during the reaction time. The mixture was diluted with $\mathrm{Et}_{2} \mathrm{O}(100 \mathrm{~mL})$ and poured into a solution of $\mathrm{Na}_{2} \mathrm{~S}_{2} \mathrm{O}_{3}(17 \mathrm{~g})$ in $80 \%$ saturated $\mathrm{NaHCO}_{3}(100 \mathrm{~mL})$. After stirring for $10 \mathrm{~min}$, the layers were separated and the aqueous layer was extracted with $\mathrm{Et}_{2} \mathrm{O}(100 \mathrm{~mL})$. The combined organic layers were washed with ice-cold saturated $\mathrm{NaHCO}_{3}(30 \mathrm{~mL})$ and water $(30 \mathrm{~mL})$. The solution was dried $\left(\mathrm{Na}_{2} \mathrm{SO}_{4}\right)$ and concentrated under reduced pressure to give the crude aldehyde 7 . To a solution of 4bromoaniline (6a) $(1.72 \mathrm{~g}, 10.0 \mathrm{mmol})$ in dichloroethane $(60 \mathrm{~mL})$ was added the above crude aldehyde, followed by $\mathrm{NaBH}(\mathrm{OAc})_{3}(4.24 \mathrm{~g}, 20.0 \mathrm{mmol})$. The mixture was stirred at room temperature overnight. Saturated $\mathrm{NaHCO}_{3}(20 \mathrm{~mL})$ was added and the layers were separated. The aqueous layer was extracted with $\mathrm{CH}_{2} \mathrm{Cl}_{2}(2 \times 30 \mathrm{~mL})$. The combined organic layers were washed with brine $(3 \times 30 \mathrm{~mL})$, dried $\left(\mathrm{Na}_{2} \mathrm{SO}_{4}\right)$, and concentrated under reduced pressure. Flash column chromatography of the crude product on silica gel using 0 $30 \%$ EtOAc in hexanes afforded $8 \mathbf{a}(2.78 \mathrm{~g}, 75 \%)$ as a white solid: $\mathrm{mp} 103-105{ }^{\circ} \mathrm{C} ;{ }^{1} \mathrm{H}$ NMR $\left(300 \mathrm{MHz} ; \mathrm{CDCl}_{3}\right) \delta 7.24(\mathrm{~d}, J=9.0 \mathrm{~Hz}, 2 \mathrm{H}), 6.45(\mathrm{~d}, J=9.0 \mathrm{~Hz}, 2 \mathrm{H}), 4.52(\mathrm{~d}, J=$ $9.0 \mathrm{~Hz}, 1 \mathrm{H}), 4.20$ (br s, 1H), 3.82-3.65 (m, 1H), 3.30-3.14 (m, 1H), 3.05-2.89 (m, 1H), $1.60-1.47(\mathrm{~m}, 1 \mathrm{H}), 1.44(\mathrm{~s}, 9 \mathrm{H}), 1.23-1.10(\mathrm{~m}, 1 \mathrm{H}), 0.95(\mathrm{~d}, J=6.0 \mathrm{~Hz}, 3 \mathrm{H}), 0.95(\mathrm{t}, J=7.5$ $\mathrm{Hz}, 3 \mathrm{H}) ;{ }^{13} \mathrm{C} \mathrm{NMR}\left(75 \mathrm{MHz} ; \mathrm{CDCl}_{3}\right) \delta 154.9,145.7,130.1,112.4,106.9,77.9,52.9,45.0$, 35.7, 26.6, 23.6, 13.8, 9.9; MS (ESI) $\mathrm{m} / z 371.3[\mathrm{M}+\mathrm{H}]^{+}\left({ }^{79} \mathrm{Br}\right), 373.3[\mathrm{M}+\mathrm{H}]^{+}\left({ }^{81} \mathrm{Br}\right)$.

tert-Butyl [(2S,3S)-1-Phenylamino-3-methylpentan-2-yl]-carbamate (8b)-The procedure for $8 \mathbf{a}$ was followed using $140 \mathrm{mg}(1.5 \mathrm{mmol})$ of $\mathbf{6 b}$ to give $336 \mathrm{mg}(77 \%)$ of $\mathbf{8 b}$ as a white solid: $\mathrm{mp} 77-79{ }^{\circ} \mathrm{C} ;{ }^{1} \mathrm{H} \mathrm{NMR}\left(300 \mathrm{MHz} ; \mathrm{CDCl}_{3}\right) \delta 7.16(\mathrm{t}, J=7.5 \mathrm{~Hz}, 2 \mathrm{H}), 6.69$ $(\mathrm{t}, J=7.5 \mathrm{~Hz}, 1 \mathrm{H}), 6.59(\mathrm{~d}, J=9.0 \mathrm{~Hz}, 2 \mathrm{H}), 4.57$ (d, $J=9.0 \mathrm{~Hz}, 1 \mathrm{H}), 4.13$ (br s, $1 \mathrm{H})$, $3.82-3.68(\mathrm{~m}, 1 \mathrm{H}), 3.26(\mathrm{dd}, J=12.0,3.0 \mathrm{~Hz}, 1 \mathrm{H}), 3.02(\mathrm{t}, J=10.5 \mathrm{~Hz}, 1 \mathrm{H}), 1.68-1.46(\mathrm{~m}$, $1 \mathrm{H}), 1.45(\mathrm{~s}, 9 \mathrm{H}), 1.28-1.10(\mathrm{~m}, 1 \mathrm{H}), 0.98-0.89(\mathrm{~m}, 6 \mathrm{H}) ;{ }^{13} \mathrm{C} \mathrm{NMR}\left(75 \mathrm{MHz} ; \mathrm{CDCl}_{3}\right) \delta$ $156.6,148.3,129.2,117.4,112.8,79.5,54.7,46.6,37.5,28.4,25.4,15.5,11.7$; MS (ESI) $\mathrm{m} / \mathrm{z} 293.3[\mathrm{M}+\mathrm{H}]^{+}$.

tert-Butyl \{(2S,3S)-1-[(4-Hexylphenyl)amino]-3-methylpentan-2-yl\}carbamate (8c)-The procedure for $\mathbf{8 a}$ was followed using $355 \mathrm{mg}(2.0 \mathrm{mmol})$ of $\mathbf{6 c}$ to give $526 \mathrm{mg}$ $(70 \%)$ of $8 \mathrm{c}$ as an oil: ${ }^{1} \mathrm{H} \mathrm{NMR}\left(300 \mathrm{MHz} ; \mathrm{CDCl}_{3}\right) \delta 6.98(\mathrm{~d}, J=9.0 \mathrm{~Hz}, 2 \mathrm{H}), 6.53(\mathrm{~d}, J=$ $9.0 \mathrm{~Hz}, 2 \mathrm{H}), 4.52$ (d, $J=9.0 \mathrm{~Hz}, 1 \mathrm{H}), 3.86$ (br s, 1H), 3.80-3.66 (m, 1H), 3.30-3.18 (m, 
$1 \mathrm{H}), 3.06-2.90(\mathrm{~m}, 1 \mathrm{H}), 2.48(\mathrm{t}, J=6.0 \mathrm{~Hz}, 2 \mathrm{H}), 1.70-1.53(\mathrm{~m}, 2 \mathrm{H}), 1.44(\mathrm{~s}, 9 \mathrm{H}), 1.38-1.10$ $(\mathrm{m}, 8 \mathrm{H}), 1.00-0.80(\mathrm{~m}, 9 \mathrm{H}) ;{ }^{13} \mathrm{C} \mathrm{NMR}\left(75 \mathrm{MHz} ; \mathrm{CDCl}_{3}\right) \delta 156.5,146.5,131.8,129.1$, $112.8,79.4,54.8,46.7,37.5,35.1,31.9,31.8,29.0,28.4,25.4,22.7,15.5,14.1,11.7$; MS (ESI) $\mathrm{m} / \mathrm{z} 377.3[\mathrm{M}+\mathrm{H}]^{+}$.

tert-Butyl \{(2S,3S)-1-[(4-Cyclohexylphenyl)amino]-3-methylpentan-2yl\}carbamate (8d)-The procedure for 8a was followed using $263 \mathrm{mg}(1.5 \mathrm{mmol})$ of $\mathbf{6 d}$ to give $345 \mathrm{mg}(61 \%)$ of $\mathbf{8 d}$ as a white solid: $\mathrm{mp} 108-110{ }^{\circ} \mathrm{C}$; ${ }^{1} \mathrm{H}$ NMR $\left(300 \mathrm{MHz} ; \mathrm{CDCl}_{3}\right)$ $\delta 7.02(\mathrm{~d}, J=9.0 \mathrm{~Hz}, 2 \mathrm{H}), 6.55(\mathrm{~d}, J=9.0 \mathrm{~Hz}, 2 \mathrm{H}), 4.53(\mathrm{~d}, J=9.0 \mathrm{~Hz}, 1 \mathrm{H}), 4.00(\mathrm{br} \mathrm{s}, 1 \mathrm{H})$, 3.80-3.66 (m, 1H), 3.32-3.20 (m, 1H), 3.10-2.90 (m, 1H), 2.46-2.31 (m, 1H), 1.90-1.70 $(\mathrm{m}, 5 \mathrm{H}), 1.70-1.10(\mathrm{~m}, 16 \mathrm{H}), 0.98-0.90(\mathrm{~m}, 6 \mathrm{H}) ;{ }^{13} \mathrm{C} \mathrm{NMR}\left(75 \mathrm{MHz} ; \mathrm{CDCl}_{3}\right) \delta 156.5$, 146.5, 137.3, 127.5, 122.8, 79.4, 54.8, 46.7, 43.7, 37.4, 34.8, 28.4, 27.1, 26.3, 25.4, 15.5, 11.7; MS (ESI) $\mathrm{m} / \mathrm{z} 375.2[\mathrm{M}+\mathrm{H}]^{+}$.

tert-Butyl [(2S,3S)-1-Biphenylamino-3-methylpentan-2-yl]-carbamate (8e)-The procedure for $8 \mathbf{a}$ was followed using $545 \mathrm{mg}(2.5 \mathrm{mmol})$ of 6e to give $560 \mathrm{mg}(76 \%)$ of $8 \mathrm{e}$ as a white solid: mp $98-100{ }^{\circ} \mathrm{C} ;{ }^{1} \mathrm{H}$ NMR $\left(300 \mathrm{MHz} ; \mathrm{CDCl}_{3}\right) \delta 7.56-7.50(\mathrm{~m}, 2 \mathrm{H})$, 7.46-7.34 (m, 4H), 7.28-7.21 (m, 1H), $6.66(\mathrm{~d}, J=9.0 \mathrm{~Hz}, 2 \mathrm{H}), 4.47(\mathrm{~d}, J=9.0 \mathrm{~Hz}, 1 \mathrm{H})$, 4.18 (br s, 1H), 3.81-3.71 (m, 1H), 3.32-3.22 (m, 1H), 3.10-2.96 (m, 1H), 1.68-1.46 (m, $1 \mathrm{H}), 1.45(\mathrm{~s}, 9 \mathrm{H}), 1.30-1.10(\mathrm{~m}, 1 \mathrm{H}), 0.98(\mathrm{~d}, J=9.0 \mathrm{~Hz}, 3 \mathrm{H}), 0.95(\mathrm{t}, J=7.5 \mathrm{~Hz}, 3 \mathrm{H}) ;{ }^{13} \mathrm{C}$ NMR (75 MHz; $\left.\mathrm{CDCl}_{3}\right) \delta 156.7,148.1,141.4,130.2,128.7,127.6,126.6,126.1,133.0$, 79.6, 54.8, 46.6, 37.5, 28.5, 25.5, 15.6, 11.7; MS (ESI) m/z 369.4 [M + H] $]^{+}$.

\section{tert-Butyl \{(2S,3S)-1-[(4-Phenoxyphenyl)amino]-3-methylpentan-2-}

yl\}carbamate (8f)-The procedure for $\mathbf{8 a}$ was followed using $370 \mathrm{mg}(2.0 \mathrm{mmol})$ of $\mathbf{6} \mathbf{f}$ to give $350 \mathrm{mg}(46 \%)$ of $\mathbf{8 f}$ as an oil: ${ }^{1} \mathrm{H}$ NMR $\left(300 \mathrm{MHz} ; \mathrm{CDCl}_{3}\right) \delta 7.32-7.25(\mathrm{~m}, 2 \mathrm{H})$, 7.05-6.98 (m, 1H), 6.96-6.86 (m, 4H), $6.59(\mathrm{~d}, J=9.0 \mathrm{~Hz}, 2 \mathrm{H}), 4.56(\mathrm{~d}, J=9.0 \mathrm{~Hz}, 1 \mathrm{H})$, 4.02 (br s, 1H), 3.81-3.72 (m, 1H), 3.31-3.20 (m, 1H), 3.06-2.95 (m, 1H), 1.66-1.50 (m, $1 \mathrm{H}), 1.45(\mathrm{~s}, 9 \mathrm{H}), 1.26-1.10(\mathrm{~m}, 1 \mathrm{H}), 1.02-0.90(\mathrm{~m}, 6 \mathrm{H}) ;{ }^{13} \mathrm{C} \mathrm{NMR}\left(75 \mathrm{MHz} ; \mathrm{CDCl}_{3}\right) \delta$ $159.3,156.7,147.5,145.3,129.9,121.9,121.3,117.1,113.7,79.4,54.8,46.9,37.5,28.5$, 25.4, 15.6, 11.7; MS (ESI) $\mathrm{m} / \mathrm{z} 385.4[\mathrm{M}+\mathrm{H}]^{+}$.

tert-Butyl \{(2S,3S)-1-[(4-Benzylphenyl)amino]-3-methylpentan-2-yl\}carbamate $(\mathbf{8 g})$ - The procedure for $\mathbf{8 a}$ was followed using $275 \mathrm{mg}(1.5 \mathrm{mmol})$ of $\mathbf{6 g}$ to give $390 \mathrm{mg}$ (68\%) of $\mathbf{8 g}$ as an off-white solid: $\mathrm{mp} 65-66{ }^{\circ} \mathrm{C} ;{ }^{1} \mathrm{H}$ NMR $\left(300 \mathrm{MHz} ; \mathrm{CDCl}_{3}\right) \delta 7.30-7.15$ (m, 5H), $6.99(\mathrm{~d}, J=9.0 \mathrm{~Hz}, 2 \mathrm{H}), 6.54(\mathrm{~d}, J=9.0 \mathrm{~Hz}, 2 \mathrm{H}), 4.54(\mathrm{~d}, J=9.0 \mathrm{~Hz}, 1 \mathrm{H}), 3.95(\mathrm{br}$ $\mathrm{s}, 1 \mathrm{H}), 3.87(\mathrm{~s}, 2 \mathrm{H}), 3.83-3.70(\mathrm{~m}, 1 \mathrm{H}), 3.30-3.18(\mathrm{~m}, 1 \mathrm{H}), 3.06-2.95(\mathrm{~m}, 1 \mathrm{H}), 1.65-1.50$ (m, 1H), 1.44 (s, 9H), 1.28-1.10 (m, 1H), 1.01-0.90 (m, 6H); $\left.{ }^{13} \mathrm{C} \mathrm{NMR} \mathrm{(75} \mathrm{MHz;} \mathrm{CDCl}_{3}\right) \delta$ 156.5, 146.6, 142.1, 130.0, 129.7, 128.8, 128.3, 125.8, 113.0, 79.5, 54.7, 46.8, 41.1, 37.5, 28.4, 25.4, 15.5, 11.7; MS (ESI) $\mathrm{m} / \mathrm{z} 383.5[\mathrm{M}+\mathrm{H}]^{+}$.

tert-Butyl \{(2S,3S)-1-[(4-Phenethylphenyl)amino]-3-methylpentan-2yl\}carbamate (8h)-The procedure for $8 \mathbf{a}$ was followed using $290 \mathrm{mg}(1.5 \mathrm{mmol})$ of $\mathbf{6 h}$ to give $325 \mathrm{mg}(55 \%)$ of $\mathbf{8 h}$ as a white solid: $\mathrm{mp} 55-57{ }^{\circ} \mathrm{C} ;{ }^{1} \mathrm{H} \mathrm{NMR}\left(300 \mathrm{MHz} ; \mathrm{CDCl}_{3}\right) \delta$ $7.30-7.10(\mathrm{~m}, 5 \mathrm{H}), 6.99$ (d, $J=9.0 \mathrm{~Hz}, 2 \mathrm{H}), 6.59$ (d, $J=9.0 \mathrm{~Hz}, 2 \mathrm{H}), 4.62(\mathrm{~d}, J=9.0 \mathrm{~Hz}$, 
1H), 3.95 (br s, 1H), 3.80-3.68 (m, 1H), $3.25(\mathrm{dd}, J=12.0,3.0 \mathrm{~Hz}, 1 \mathrm{H}), 3.02(\mathrm{t}, J=10.5 \mathrm{~Hz}$, $1 \mathrm{H}), 2.90-2.76(, 4 \mathrm{H}), 1.65-1.50(\mathrm{~m}, 1 \mathrm{H}), 1.44(\mathrm{~s}, 9 \mathrm{H}), 1.23-1.10(\mathrm{~m}, 1 \mathrm{H}), 0.98-0.88(\mathrm{~m}$, $6 \mathrm{H}) ;{ }^{13} \mathrm{C} \mathrm{NMR}\left(75 \mathrm{MHz} ; \mathrm{CDCl}_{3}\right) \delta 156.6,145.9,142.1,131.4,129.2,128.5,128.3,125.8$, $113.5,79.5,54.6,47.2,38.3,37.5,37.1,28.4,25.4,15.5,11.7$; MS (ESI) $\mathrm{m} / \mathrm{z} 397.5[\mathrm{M}+$ $\mathrm{H}]^{+}$.

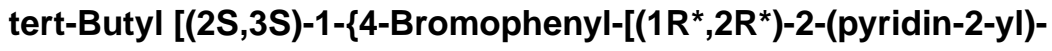
cyclopropanecarbonyl]amino\}-3-methylpentan-2-yl]carbamate (9a)-To a solution of ( \pm )-trans-2-(pyridin-2-yl)-cyclopropanecarboxylic acid $(0.40 \mathrm{~g}, 2.0 \mathrm{mmol})$ in $\mathrm{CH}_{2} \mathrm{Cl}_{2}(20 \mathrm{~mL})$ at room temperature was added oxalyl chloride $(0.35 \mathrm{~mL}, 4.0 \mathrm{mmol})$ and DMF $(50 \mu \mathrm{L})$. The mixture was stirred at $40{ }^{\circ} \mathrm{C}$ for $2 \mathrm{~h}$, then cooled to room temperature and concentrated under reduced pressure. The residue was dissolved in $\mathrm{CH}_{2} \mathrm{Cl}_{2}(20 \mathrm{~mL})$ and treated with $8 \mathbf{a}(0.74 \mathrm{~g}, 2.0 \mathrm{mmol})$ and $\mathrm{Et}_{3} \mathrm{~N}(1.1 \mathrm{~mL}, 8.0 \mathrm{mmol})$. The resulting solution was stirred at room temperature overnight. Saturated $\mathrm{NaHCO}_{3}(10 \mathrm{~mL})$ was added and the layers were separated. The aqueous layer was extracted with $\mathrm{CH}_{2} \mathrm{Cl}_{2}(3 \times 10 \mathrm{~mL})$. The combined organic layers were washed with brine $(3 \times 20 \mathrm{~mL})$, dried $\left(\mathrm{Na}_{2} \mathrm{SO}_{4}\right)$ and concentrated under reduced pressure. Flash column chromatography of the crude product on silica gel using 0 $25 \%$ EtOAc in hexanes afforded 9a $(0.74 \mathrm{~g}, 72 \%, 1: 1$ diastereomeric mixture) as a light yellow foam: ${ }^{1} \mathrm{H}$ NMR $\left(300 \mathrm{MHz} ; \mathrm{CDCl}_{3}\right) \delta 8.30(\mathrm{~d}, J=6.0 \mathrm{~Hz}, 1 \mathrm{H}), 7.58-7.34(\mathrm{~m}, 3 \mathrm{H})$, $7.22-6.98(\mathrm{~m}, 4 \mathrm{H}), 4.96(\mathrm{~d}, J=9.0 \mathrm{~Hz}, 1 \mathrm{H}), 4.45-4.25(\mathrm{~m}, 1 \mathrm{H}), 3.80-3.78(\mathrm{~m}, 1 \mathrm{H})$, $3.21-3.06(\mathrm{~m}, 1 \mathrm{H}), 2.71-2.62(\mathrm{~m}, 0.5 \mathrm{H}), 2.58-2.48(\mathrm{~m}, 0.5 \mathrm{H}), 1.98-1.86(\mathrm{~m}, 1 \mathrm{H})$, $1.75-1.62(\mathrm{~m}, 1 \mathrm{H}), 1.60-1.35(\mathrm{~m}, 3 \mathrm{H}), 1.45$ and $1.40(2 \mathrm{~s}, 9 \mathrm{H}), 1.15-1.02(\mathrm{~m}, 1 \mathrm{H})$, 0.92-0.80 (m, 6H); MS (ESI) $\left.\mathrm{m} / \mathrm{z} 516.7[\mathrm{M}+\mathrm{H}]{ }^{+}\left({ }^{79} \mathrm{Br}\right), 518.6[\mathrm{M}+\mathrm{H}]\right]^{+}\left({ }^{81} \mathrm{Br}\right)$.

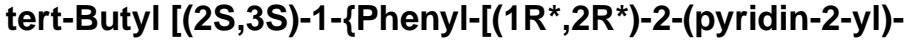
cyclopropanecarbonyl]amino\}-3-methylpentan-2-yl]carbamate (9b)-The procedure for $\mathbf{9 a}$ was followed using $102 \mathrm{mg}(0.35 \mathrm{mmol})$ of $\mathbf{8 b}$ to give $85 \mathrm{mg}(56 \%)$ of $\mathbf{9 b}$ (1:1 diastereomeric mixture) as an oil: ${ }^{1} \mathrm{H}$ NMR $\left(300 \mathrm{MHz} ; \mathrm{CDCl}_{3}\right) \delta 8.26(\mathrm{~d}, J=6.0 \mathrm{~Hz}$, $1 \mathrm{H}), 7.54-7.46(\mathrm{~m}, 1 \mathrm{H}), 7.38-7.10(\mathrm{~m}, 6 \mathrm{H}), 7.03-6.96(\mathrm{~m}, 1 \mathrm{H}), 4.96(\mathrm{t}, J=7.5 \mathrm{~Hz}, 1 \mathrm{H})$, $4.48-4.32(\mathrm{~m}, 1 \mathrm{H}), 3.81-3.62(\mathrm{~m}, 1 \mathrm{H}), 3.22-3.08(\mathrm{~m}, 1 \mathrm{H}), 2.72-2.62(\mathrm{~m}, 0.5 \mathrm{H}), 2.58-2.48$ $(\mathrm{m}, 0.5 \mathrm{H}), 2.00-1.88(\mathrm{~m}, 1 \mathrm{H}), 1.72-1.48(\mathrm{~m}, 4 \mathrm{H}), 1.46$ and $1.42(2 \mathrm{~s}, 9 \mathrm{H}), 1.15-1.00(\mathrm{~m}$, $1 \mathrm{H}), 0.91-0.78(\mathrm{~m}, 6 \mathrm{H})$; MS (ESI) $\mathrm{m} / \mathrm{z} 438.5[\mathrm{M}+\mathrm{H}]^{+}$.

\section{tert-Butyl [(2S,3S)-1-\{4-Hexylphenyl-[(1 $\left.{ }^{\star}, 2 R^{\star}\right)-2-($ pyridin-2-yl)- cyclopropanecarbonyl]amino\}-3-methylpentan-2-yl]carbamate (9c)-The} procedure for $\mathbf{9 a}$ was followed using $188 \mathrm{mg}(0.5 \mathrm{mmol})$ of $\mathbf{8 c}$ to give $140 \mathrm{mg}(54 \%)$ of $\mathbf{9 c}$ (1:1 diastereomeric mixture) as an oil: ${ }^{1} \mathrm{H}$ NMR $\left(300 \mathrm{MHz} ; \mathrm{CDCl}_{3}\right) \delta 8.28-8.22(\mathrm{~m}, 1 \mathrm{H})$, 7.53-7.45 (m, 1H), 7.20-6.92 (m, 6H), $5.10(\mathrm{t}, J=9.0 \mathrm{~Hz}, 1 \mathrm{H}), 4.45-4.31(\mathrm{~m}, 1 \mathrm{H})$, $3.80-3.60(\mathrm{~m}, 1 \mathrm{H}), 3.18-3.06(\mathrm{~m}, 1 \mathrm{H}), 2.70-2.61(\mathrm{~m}, 0.5 \mathrm{H}), 2.60-2.47(\mathrm{~m}, 2.5 \mathrm{H})$, 2.00-1.89 (m, 1H), 1.71-1.30 (m, 24H), 1.20-1.02 (m, 1H), 0.96-0.80 (m, 6H); MS (ESI) $\mathrm{m} / \mathrm{z} 522.6[\mathrm{M}+\mathrm{H}]^{+}$.

\section{tert-Butyl [(2S,3S)-1-\{4-Cyclohexylphenyl-[(1 $\left.R^{\star}, 2 R^{\star}\right)-2-($ pyridin-2-} yl)cyclopropanecarbonyl]amino\}-3-methylpentan-2-yl]carbamate (9d)—The procedure for $\mathbf{9 a}$ was followed using $131 \mathrm{mg}(0.35 \mathrm{mmol})$ of $\mathbf{8 d}$ to give $104 \mathrm{mg}(57 \%)$ of $\mathbf{9 d}$ 
(1:1 diastereomeric mixture) as an oil: ${ }^{1} \mathrm{H}$ NMR $\left(300 \mathrm{MHz} ; \mathrm{CDCl}_{3}\right) \delta 8.30-8.20(\mathrm{~m}, 1 \mathrm{H})$, $7.52-7.45(\mathrm{~m}, 1 \mathrm{H}), 7.20-6.92(\mathrm{~m}, 6 \mathrm{H}), 5.12(\mathrm{dd}, J=12.0,9.0 \mathrm{~Hz}, 1 \mathrm{H}), 4.46-4.30(\mathrm{~m}, 1 \mathrm{H})$, $3.80-3.60(\mathrm{~m}, 1 \mathrm{H}), 3.20-3.06(\mathrm{~m}, 1 \mathrm{H}), 2.70-2.60(\mathrm{~m}, 0.5 \mathrm{H}), 2.58-2.47(\mathrm{~m}, 1.5 \mathrm{H})$, $2.00-1.65(\mathrm{~m}, 7 \mathrm{H}), 1.55-1.20(\mathrm{~m}, 17 \mathrm{H}), 1.18-1.00(\mathrm{~m}, 1 \mathrm{H}), 0.92-0.78(\mathrm{~m}, 6 \mathrm{H}) ; \mathrm{MS}(\mathrm{ESI})$ $\mathrm{m} / \mathrm{z} 520.6[\mathrm{M}+\mathrm{H}]^{+}$.

tert-Butyl [(2S,3S)-1-\{Biphenyl-[(1R $\left.{ }^{\star}, 2 R^{\star}\right)-2-($ pyridin-2-yl)cyclopropanecarbonyl]amino\}-3-methylpentan-2-yl]carbamate (9e)-The procedure for $\mathbf{9 a}$ was followed using $184 \mathrm{mg}(0.5 \mathrm{mmol})$ of $8 \mathbf{e}$ to give $130 \mathrm{mg}(51 \%)$ of $\mathbf{9 e}$ (1:1 diastereomeric mixture) as an oil: ${ }^{1} \mathrm{H}$ NMR $\left(300 \mathrm{MHz} ; \mathrm{CDCl}_{3}\right) \delta 8.29-8.23(\mathrm{~m}, 1 \mathrm{H})$, $7.60-7.15(\mathrm{~m}, 11 \mathrm{H}), 7.01-6.95(\mathrm{~m}, 1 \mathrm{H}), 5.11(\mathrm{t}, J=9.0 \mathrm{~Hz}, 1 \mathrm{H}), 4.50-4.37(\mathrm{~m}, 1 \mathrm{H})$, $3.86-3.68(\mathrm{~m}, 1 \mathrm{H}), 3.26-3.17(\mathrm{~m}, 1 \mathrm{H}), 2.75-2.68(\mathrm{~m}, 0.5 \mathrm{H}), 2.60-2.51(\mathrm{~m}, 0.5 \mathrm{H})$, $2.10-1.98(\mathrm{~m}, 1 \mathrm{H}), 1.75-1.67(\mathrm{~m}, 0.5 \mathrm{H}), 1.66-1.58(\mathrm{~m}, 0.5 \mathrm{H}), 1.57-1.40(\mathrm{~m}, 3 \mathrm{H}), 1.47$ and $1.43(2 \mathrm{~s}, 9 \mathrm{H}), 1.20-1.03(\mathrm{~m}, 1 \mathrm{H}), 0.93-0.80(\mathrm{~m}, 6 \mathrm{H})$; MS (ESI) $\mathrm{m} / \mathrm{z} 514.7[\mathrm{M}+\mathrm{H}]^{+}$.

tert-Butyl [(2S,3S)-1-\{4-Phenoxyphenyl-[(1R $\left.{ }^{\star}, 2 R^{\star}\right)$-2-(pyridin-2yl)cyclopropanecarbonyl]amino\}-3-methylpentan-2-yl]carbamate (9f)-The procedure for $\mathbf{9 a}$ was followed using $130 \mathrm{mg}(0.34 \mathrm{mmol})$ of $\mathbf{8 f}$ to give $57 \mathrm{mg}(32 \%)$ of $\mathbf{9 f}$ (1:1 diastereomeric mixture) as an oil: ${ }^{1} \mathrm{H}$ NMR $\left(300 \mathrm{MHz} ; \mathrm{CDCl}_{3}\right) \delta 8.33-8.27(\mathrm{~m}, 1 \mathrm{H})$, 7.52-7.43 (m, 1H), 7.40-7.30 (m, 2H), 7.22-7.10 (m, 4H), 7.02-6.78 (m, 5H), $5.08(\mathrm{t}, J=$ $9.0 \mathrm{~Hz}, 1 \mathrm{H}), 4.55-4.40(\mathrm{~m}, 1 \mathrm{H}), 3.82-3.66(\mathrm{~m}, 1 \mathrm{H}), 3.20-3.02(\mathrm{~m}, 1 \mathrm{H}), 2.68-2.60(\mathrm{~m}$, $0.5 \mathrm{H}), 2.52-2.43(\mathrm{~m}, 0.5 \mathrm{H}), 2.02-1.90(\mathrm{~m}, 1 \mathrm{H}), 1.75-1.45(\mathrm{~m}, 4 \mathrm{H}), 1.44$ and $1.41(2 \mathrm{~s}, 9 \mathrm{H})$, $1.18-1.00(\mathrm{~m}, 1 \mathrm{H}), 0.92-0.80(\mathrm{~m}, 6 \mathrm{H})$; MS (ESI) $\mathrm{m} / \mathrm{z} 530.7[\mathrm{M}+\mathrm{H}]^{+}$.

tert-Butyl [(2S,3S)-1-\{4-Benzylphenyl-[(1 $\left.{ }^{\star}, 2 R^{\star}\right)$-2-(pyridin-2-yl)cyclopropanecarbonyl]amino\}-3-methylpentan-2-yl]carbamate (9g)-The procedure for $9 \mathbf{a}$ was followed using $100 \mathrm{mg}(0.26 \mathrm{mmol})$ of $8 \mathrm{~g}$ to give $65 \mathrm{mg}(47 \%)$ of $\mathbf{9 g}$ (1:1 diastereomeric mixture) as an oil: ${ }^{1} \mathrm{H}$ NMR $\left(300 \mathrm{MHz} ; \mathrm{CDCl}_{3}\right) \delta 8.30-8.26(\mathrm{~m}, 1 \mathrm{H})$, $7.60-7.52(\mathrm{~m}, 1 \mathrm{H}), 7.38-6.95(\mathrm{~m}, 11 \mathrm{H}), 5.06(\mathrm{t}, J=9.0 \mathrm{~Hz}, 1 \mathrm{H}), 4.48-4.30(\mathrm{~m}, 1 \mathrm{H}), 3.92$ and $3.88(2 \mathrm{~s}, 2 \mathrm{H}), 3.80-3.66(\mathrm{~m}, 1 \mathrm{H}), 3.20-3.05(\mathrm{~m}, 1 \mathrm{H}), 2.70-2.62(\mathrm{~m}, 0.5 \mathrm{H}), 2.50-2.42$ $(\mathrm{m}, 0.5 \mathrm{H}), 1.98-1.90(\mathrm{~m}, 1 \mathrm{H}), 1.76-1.46(\mathrm{~m}, 4 \mathrm{H}), 1.44$ and $1.42(2 \mathrm{~s}, 9 \mathrm{H}), 1.20-1.02(\mathrm{~m}$, $1 \mathrm{H}), 0.93-0.82(\mathrm{~m}, 6 \mathrm{H})$; MS (ESI) $\mathrm{m} / \mathrm{z} 528.8[\mathrm{M}+\mathrm{H}]^{+}$.

tert-Butyl [(2S,3S)-1-\{4-Phenethylphenyl-[(1 $\left.{ }^{\star}, 2 R^{\star}\right)$-2-(pyridin-2yl)cyclopropanecarbonyl]amino\}-3-methylpentan-2-yl]carbamate (9h)-The procedure for $\mathbf{9 a}$ was followed using $139 \mathrm{mg}(0.35 \mathrm{mmol})$ of $\mathbf{8 h}$ to give $96 \mathrm{mg}(51 \%)$ of $\mathbf{9 h}$ (1:1 diastereomeric mixture) as an oil: ${ }^{1} \mathrm{H}$ NMR $\left(300 \mathrm{MHz} ; \mathrm{CDCl}_{3}\right) \delta 8.30-8.25(\mathrm{~m}, 1 \mathrm{H})$, $7.55-7.46(\mathrm{~m}, 1 \mathrm{H}), 7.30-6.96(\mathrm{~m}, 11 \mathrm{H}), 5.10(\mathrm{dd}, J=9.0,3.0 \mathrm{~Hz}, 1 \mathrm{H}), 4.46-4.32(\mathrm{~m}, 1 \mathrm{H})$, 3.80-3.62 (m, 1H), 3.20-3.06 (m, 1H), 2.90-2.80 (m, 4H), 2.71-2.632 (m, 0.5H), 2.56-2.47 (m, 0.5H), 1.98-1.90 (m, 1H), 1.72-1.47 (m, 4H), 1.46 and $1.42(2 \mathrm{~s}, 9 \mathrm{H})$, 1.16-1.02 (m, 1H), 0.90-0.78 (m, 6H); MS (ESI) $\mathrm{m} / z 528.8[\mathrm{M}+\mathrm{H}]^{+}$.

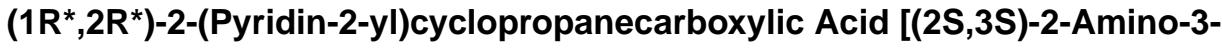
methylpentyl]-(phenyl-4-yl)amide (4a)—A solution of $9 \mathbf{b}(85 \mathrm{mg}, 0.19 \mathrm{mmol})$ and $4 \mathrm{M}$ $\mathrm{HCl}$ in dioxane $(2 \mathrm{~mL})$ in $\mathrm{CH}_{2} \mathrm{Cl}_{2}(5 \mathrm{~mL})$ was stirred at room temperature for $6 \mathrm{~h}$. The 
solvent was removed under reduced pressure. The resulting residue was triturated with hexanes to give 4a dihydrochloride (76 mg, 96\%, 1:1 diastereomeric mixture) as an offwhite solid: ${ }^{1} \mathrm{H}$ NMR (300 MHz; $\left.\mathrm{CD}_{3} \mathrm{OD}\right) \delta 8.66-8.56(\mathrm{~m}, 1 \mathrm{H}), 8.40-8.28(\mathrm{~m}, 1 \mathrm{H})$, $7.85-7.74(\mathrm{~m}, 1 \mathrm{H}), 7.61-7.33(\mathrm{~m}, 6 \mathrm{H}), 4.40(\mathrm{dd}, J=15.0,9.0 \mathrm{~Hz}, 0.5 \mathrm{H}), 4.29$ (dd, $J=15.0$, $9.0 \mathrm{~Hz}, 0.5 \mathrm{H}), 3.78-3.56(\mathrm{~m}, 1.5 \mathrm{H}), 3.39-3.30(\mathrm{~m}, 0.5 \mathrm{H}), 3.08-2.99(\mathrm{~m}, 0.5 \mathrm{H}), 2.98-2.89$ (m, 0.5H), 2.11-1.84 (m, 2H), 1.82-1.58 (m, 2H), 1.40-1.10 (m, 2H), 1.00-0.76 (m, 6H); HRMS (ESI) calcd for $\mathrm{C}_{21} \mathrm{H}_{27} \mathrm{~N}_{3} \mathrm{O}[\mathrm{M}+\mathrm{H}]^{+}: 338.2227$. Found: 338.2241.

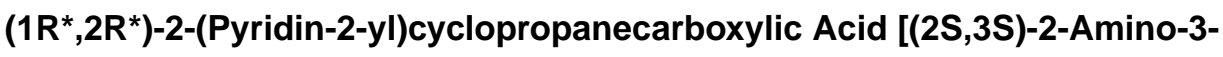
methylpentyl]-(4' -hexylphenyl-4-yl)amide (4b)-The procedure for $\mathbf{4 a}$ was followed using $135 \mathrm{mg}(0.26 \mathrm{mmol})$ of $\mathbf{9 c}$ to give $121 \mathrm{mg}(95 \%)$ of $\mathbf{4 b}$ dihydrochloride as a 1:1 diastereomeric mixture: ${ }^{1} \mathrm{H}$ NMR $\left(300 \mathrm{MHz} ; \mathrm{CD}_{3} \mathrm{OD}\right) \delta 8.26-8.16(\mathrm{~m}, 1 \mathrm{H}), 7.70-7.56(\mathrm{~m}$, $1 \mathrm{H}), 7.28-7.08(\mathrm{~m}, 6 \mathrm{H}), 4.32-4.16(\mathrm{~m}, 1 \mathrm{H}), 3.70-3.58(\mathrm{~m}, 1 \mathrm{H}), 3.36-3.20(\mathrm{~m}, 1 \mathrm{H})$, 2.66-2.50 (m, 3H), 1.92-1.80 (m, 1H), 1.78-1.48 (m, 4H), 1.48-1.10 (m, 9H), 1.00-0.76 (m, 9H); HRMS (ESI) calcd for $\mathrm{C}_{27} \mathrm{H}_{39} \mathrm{~N}_{3} \mathrm{O}[\mathrm{M}+\mathrm{H}]^{+}:$422.3166. Found: 422.3170.

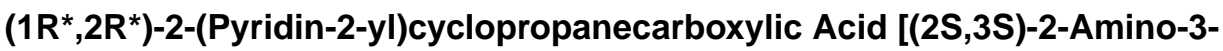
methylpentyl]-(4' -cyclohexylphenyl-4-yl)amide (4c)-The procedure for $4 \mathbf{a}$ was followed using $104 \mathrm{mg}(0.2 \mathrm{mmol})$ of $\mathbf{9 d}$ to give $93 \mathrm{mg}(95 \%)$ of $\mathbf{4 c}$ dihydrochloride as a 1:1 diastereomeric mixture: ${ }^{1} \mathrm{H}$ NMR $\left(300 \mathrm{MHz} ; \mathrm{CD}_{3} \mathrm{OD}\right) \delta 8.63-8.52(\mathrm{~m}, 1 \mathrm{H}), 8.38-8.24(\mathrm{~m}$, $1 \mathrm{H}), 7.85-7.72(\mathrm{~m}, 1 \mathrm{H}), 7.60-7.48(\mathrm{~m}, 1 \mathrm{H}), 7.48-7.20(\mathrm{~m}, 4 \mathrm{H}), 4.36(\mathrm{dd}, J=15.0,9.0 \mathrm{~Hz}$, $0.5 \mathrm{H}), 4.25(\mathrm{dd}, J=15.0,9.0 \mathrm{~Hz}, 0.5 \mathrm{H}), 3.78-3.56(\mathrm{~m}, 1.5 \mathrm{H}), 3.40-3.30(\mathrm{~m}, 0.5 \mathrm{H})$, $3.04-2.95(\mathrm{~m}, 0.5 \mathrm{H}), 2.94-2.83(\mathrm{~m}, 0.5 \mathrm{H}), 2.60-2.42(\mathrm{~m}, 1 \mathrm{H}), 2.10-2.00(\mathrm{~m}, 1 \mathrm{H})$, $1.98-1.52(\mathrm{~m}, 7 \mathrm{H}), 1.52-1.05(\mathrm{~m}, 8 \mathrm{H}), 1.00-0.76(\mathrm{~m}, 6 \mathrm{H})$; HRMS (ESI) calcd for $\mathrm{C}_{27} \mathrm{H}_{37} \mathrm{~N}_{3} \mathrm{O}[\mathrm{M}+\mathrm{H}]^{+}:$420.3009. Found: 420.3022 .

$\left(1 R^{\star}, 2 R^{\star}\right)$-2-(Pyridin-2-yl)cyclopropanecarboxylic Acid [(2S,3S)-2-Amino-3methylpentyl]-(biphenyl-4-yl)amide (4d)—The procedure for $4 \mathbf{a}$ was followed using $125 \mathrm{mg}(0.24 \mathrm{mmol})$ of $\mathbf{9 e}$ to give $115 \mathrm{mg}(97 \%)$ of $\mathbf{4 d}$ dihydrochloride as a 1:1 diastereomeric mixture: ${ }^{1} \mathrm{H}$ NMR $\left(300 \mathrm{MHz} ; \mathrm{CD}_{3} \mathrm{OD}\right) \delta 8.28-8.21(\mathrm{~m}, 1 \mathrm{H}), 7.72-7.52(\mathrm{~m}$, $5 \mathrm{H}), 7.48-7.32(\mathrm{~m}, 5 \mathrm{H}), 7.30-7.23(\mathrm{~m}, 1 \mathrm{H}), 7.19-7.10(\mathrm{~m}, 1 \mathrm{H}), 4.40-4.22(\mathrm{~m}, 1 \mathrm{H})$, 3.80-3.66 (m, 1H), 3.40-3.22 (m, 1H), 2.70-2.53 (m, 1H), 2.05-1.90 (m, 1H), 1.84-1.62 (m, 2H), 1.50-1.15 (m, 3H), 0.99 and $0.97(2 \mathrm{~d}, J=6.0 \mathrm{~Hz}, 3 \mathrm{H}), 0.90-0.80(\mathrm{~m}, 3 \mathrm{H})$; HRMS (ESI) calcd for $\mathrm{C}_{27} \mathrm{H}_{31} \mathrm{~N}_{3} \mathrm{O}[\mathrm{M}+\mathrm{H}]^{+}$: 414.2540. Found: 414.2541.

$\left(1 R^{\star}, 2 R^{\star}\right)$-2-(Pyridin-2-yl)cyclopropanecarboxylic Acid [(2S,3S)-2-Amino-3methylpentyl]-(4' -phenoxyphenyl-4-yl)amide (4e)—The procedure for $\mathbf{4 a}$ was followed using $50 \mathrm{mg}(0.09 \mathrm{mmol})$ of $9 \mathrm{f}$ to give $46 \mathrm{mg}(97 \%)$ of $4 \mathbf{e}$ dihydrochloride as a 1:1 diastereomeric mixture: ${ }^{1} \mathrm{H}$ NMR $\left(300 \mathrm{MHz} ; \mathrm{CD}_{3} \mathrm{OD}\right) \delta 8.60-8.46(\mathrm{~m}, 1 \mathrm{H}), 8.32-8.20(\mathrm{~m}$, $1 \mathrm{H}), 7.78-7.64(\mathrm{~m}, 1 \mathrm{H}), 7.60-7.20(\mathrm{~m}, 5 \mathrm{H}), 7.12-7.02(\mathrm{~m}, 1 \mathrm{H}), 7.00-6.76(\mathrm{~m}, 4 \mathrm{H}), 4.32$ $(\mathrm{dd}, J=15.0,9.0 \mathrm{~Hz}, 0.5 \mathrm{H}), 4.13(\mathrm{dd}, J=15.0,9.0 \mathrm{~Hz}, 0.5 \mathrm{H}), 3.68-3.40(\mathrm{~m}, 1.5 \mathrm{H})$, $3.35-3.25(\mathrm{~m}, 0.5 \mathrm{H}), 3.02-2.90(\mathrm{~m}, 0.5 \mathrm{H}), 2.88-2.78(\mathrm{~m}, 0.5 \mathrm{H}), 2.08-1.54(\mathrm{~m}, 4 \mathrm{H})$, $1.42-1.08(\mathrm{~m}, 2 \mathrm{H}), 0.98-0.68(\mathrm{~m}, 6 \mathrm{H})$; HRMS (ESI) calcd for $\mathrm{C}_{27} \mathrm{H}_{31} \mathrm{~N}_{3} \mathrm{O}[\mathrm{M}+\mathrm{H}]^{+}$: 430.2489. Found: 430.2505. 
$\left(1 R^{\star}, 2 R^{\star}\right)$-2-(Pyridin-2-yl)cyclopropanecarboxylic Acid [(2S,3S)-2-Amino-3methylpentyl]-(4' -benzylphenyl-4-yl)amide (4f)_-The procedure for $\mathbf{4 a}$ was followed using $65 \mathrm{mg}(0.12 \mathrm{mmol})$ of $\mathbf{9 g}$ to give $51 \mathrm{mg}(83 \%)$ of $\mathbf{4 f}$ dihydrochloride as a $1: 1$ diastereomeric mixture: ${ }^{1} \mathrm{H}$ NMR $\left(300 \mathrm{MHz}\right.$; $\left.\mathrm{CD}_{3} \mathrm{OD}\right) \delta 8.52-8.46(\mathrm{~m}, 1 \mathrm{H}), 8.22-8.10(\mathrm{~m}$, $1 \mathrm{H}), 7.70-7.60(\mathrm{~m}, 1 \mathrm{H}), 7.50-7.32(\mathrm{~m}, 3 \mathrm{H}), 7.30-7.06(\mathrm{~m}, 7 \mathrm{H}), 4.32(\mathrm{dd}, J=15.0,9.0 \mathrm{~Hz}$, $0.5 \mathrm{H}), 4.21(\mathrm{dd}, J=15.0,9.0 \mathrm{~Hz}, 0.5 \mathrm{H}), 3.92$ and $3.90(2 \mathrm{~s}, 2 \mathrm{H}), 3.75-3.65(\mathrm{~m}, 1.5 \mathrm{H})$, $3.38-3.26(\mathrm{~m}, 0.5 \mathrm{H}), 3.00-2.90(\mathrm{~m}, 0.5 \mathrm{H}), 2.88-2.80(\mathrm{~m}, 0.5 \mathrm{H}), 2.08-1.58(\mathrm{~m}, 4 \mathrm{H})$, 1.42-1.12 (m, 2H), 0.98-0.72 (m, 6H); HRMS (ESI) calcd for $\mathrm{C}_{28} \mathrm{H}_{33} \mathrm{~N}_{3} \mathrm{O}[\mathrm{M}+\mathrm{H}]^{+}$: 428.2696. Found: 428.2711.

$\left(1 R^{\star}, 2 R^{\star}\right)$-2-(Pyridin-2-yl)cyclopropanecarboxylic Acid [(2S,3S)-2-Amino-3methylpentyl]-(4' -phenethylphenyl-4-yl)amide (4g)-The procedure for $\mathbf{4 a}$ was followed using $96 \mathrm{mg}(0.18 \mathrm{mmol})$ of $\mathbf{9 h}$ to give $87 \mathrm{mg}(95 \%)$ of $\mathbf{4 g}$ dihydrochloride as a 1:1 diastereomeric mixture: ${ }^{1} \mathrm{H}$ NMR $\left(300 \mathrm{MHz}\right.$; $\left.\mathrm{CD}_{3} \mathrm{OD}\right) \delta 8.66-8.56(\mathrm{~m}, 1 \mathrm{H}), 8.38-8.25(\mathrm{~m}$, 1H), 7.82-7.70 (m, 1H), 7.58-7.42 (m, 1H), 7.40-7.30 (m, 2H), 7.30-7.05 (m, 7H), 4.42 (dd, $J=15.0,9.0 \mathrm{~Hz}, 0.5 \mathrm{H}), 4.27(\mathrm{dd}, J=15.0,9.0 \mathrm{~Hz}, 0.5 \mathrm{H}), 3.75-3.56(\mathrm{~m}, 1.5 \mathrm{H})$, $3.38-3.28(\mathrm{~m}, 0.5 \mathrm{H}), 3.06-2.96(\mathrm{~m}, 0.5 \mathrm{H}), 2.96-2.78(\mathrm{~m}, 4.5 \mathrm{H}), 2.10-1.82(\mathrm{~m}, 2 \mathrm{H})$, $1.80-1.58(\mathrm{~m}, 2 \mathrm{H}), 1.40-1.10(\mathrm{~m}, 2 \mathrm{H}), 1.00-0.70(\mathrm{~m}, 6 \mathrm{H})$; HRMS (ESI) calcd for $\mathrm{C}_{29} \mathrm{H}_{35} \mathrm{~N}_{3} \mathrm{O}[\mathrm{M}+\mathrm{H}]^{+}:$442.2853. Found: 442.2859 .

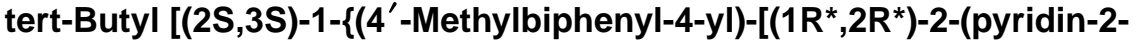 yl)cyclopropanecarbonyl]amino\}-3-methylpentan-2-yl]-carbamate (10a)—A} mixture of $9 \mathbf{a}(30 \mathrm{mg}, 0.058 \mathrm{mmol})$, 4-methylphenylboronic acid (12 mg, $0.087 \mathrm{mmol}$ ), $\mathrm{Pd}(\mathrm{dppf})-\mathrm{Cl}_{2} \cdot \mathrm{CH}_{2} \mathrm{Cl}_{2}(4.35 \mathrm{mg}, 0.0058 \mathrm{mmol})$ and $\mathrm{K}_{3} \mathrm{PO}_{4}(38 \mathrm{mg}, 2.7 \mathrm{mmol})$ in dimethoxyethane $(1 \mathrm{~mL})$ and water $(0.3 \mathrm{~mL})$ was heated in a sealed vessel by microwave irradiation at $160^{\circ} \mathrm{C}$ for $6 \mathrm{~min}$. The resulting mixture was poured into $1 \mathrm{~N} \mathrm{NaOH}$ solution (5 $\mathrm{mL})$ and extracted with $\mathrm{CH}_{2} \mathrm{Cl}_{2}(3 \times 10 \mathrm{~mL})$. The combined organic layers were dried $\left(\mathrm{Na}_{2} \mathrm{SO}_{4}\right)$ and concentrated under reduced pressure. Flash column chromatography of the crude product on silica gel using $0 \rightarrow 20 \%$ EtOAc in hexanes afforded 10a $(20 \mathrm{mg}, 65 \%)$ as a 1:1 diastereomeric mixture: ${ }^{1} \mathrm{H}$ NMR $\left(300 \mathrm{MHz} ; \mathrm{CDCl}_{3}\right) \delta 8.29-8.22(\mathrm{~m}, 1 \mathrm{H}), 7.58-7.38$ (m, 5H), 7.37-7.16 (m, 5H), 7.02-6.92 (m, 1H), 5.13-5.06 (m, 1H), 4.48-4.37 (m, 1H), $3.83-3.68(\mathrm{~m}, 1 \mathrm{H}), 3.24-3.12(\mathrm{~m}, 1 \mathrm{H}), 2.73-2.65(\mathrm{~m}, 0.5 \mathrm{H}), 2.60-2.50(\mathrm{~m}, 0.5 \mathrm{H}), 2.39(\mathrm{~s}$, $3 \mathrm{H}), 2.08-1.96(\mathrm{~m}, 1 \mathrm{H}), 1.76-1.55(\mathrm{~m}, 1 \mathrm{H}), 1.54-1.37(\mathrm{~m}, 3 \mathrm{H}), 1.47$ and $1.43(2 \mathrm{~s}, 9 \mathrm{H})$, $1.17-1.00(\mathrm{~m}, 1 \mathrm{H}), 0.92-0.78(\mathrm{~m}, 6 \mathrm{H})$; MS (ESI) $\mathrm{m} / \mathrm{z} 528.7[\mathrm{M}+\mathrm{H}]^{+}$.

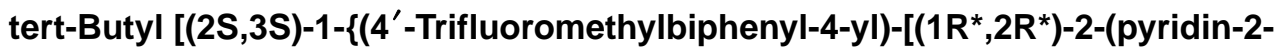
yl)cyclopropanecarbonyl]amino\}-3-methylpentan-2-yl]carbamate (10b)-The procedure for $10 \mathrm{a}$ was followed using $30 \mathrm{mg}(0.058 \mathrm{mmol})$ of $9 \mathbf{a}$ and $17 \mathrm{mg}(0.087 \mathrm{mmol})$ of 4-trifluoromethylphenylboronic acid to give $25 \mathrm{mg}$ (74\%) of $\mathbf{1 0 b}$ as a 1:1 diastereomeric mixture: ${ }^{1} \mathrm{H}$ NMR $\left(300 \mathrm{MHz} ; \mathrm{CDCl}_{3}\right) \delta 8.30-8.22(\mathrm{~m}, 1 \mathrm{H}), 7.72-7.40(\mathrm{~m}, 7 \mathrm{H}), 7.38-7.16$ (m, 3H), 7.02-6.93 (m, 1H), 5.13-5.02 (m, 1H), 4.50-4.38 (m, 1H), 3.84-3.65 (m, 1H), 3.28-3.15 (m, 1H), 2.76-2.65 (m, 0.5H), 2.61-2.48 (m, 0.5H), 2.08-1.96 (m, 1H), $1.76-1.55(\mathrm{~m}, 1 \mathrm{H}), 1.54-1.37(\mathrm{~m}, 3 \mathrm{H}), 1.47$ and $1.43(2 \mathrm{~s}, 9 \mathrm{H}), 1.18-1.00(\mathrm{~m}, 1 \mathrm{H})$, $0.94-0.78(\mathrm{~m}, 6 \mathrm{H})$; MS (ESI) $\mathrm{m} / \mathrm{z} 582.7[\mathrm{M}+\mathrm{H}]^{+}$. 


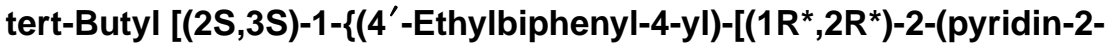
yl)cyclopropanecarbonyl]amino\}-3-methylpentan-2-yl]-carbamate (10c)-The procedure for 10a was followed using $30 \mathrm{mg}(0.058 \mathrm{mmol})$ of $9 \mathrm{a}$ and $13 \mathrm{mg}(0.087 \mathrm{mmol})$ of 4-ethylphenylboronic acid to give $25 \mathrm{mg}(80 \%)$ of $10 \mathrm{c}$ as a $1: 1$ diastereomeric mixture: ${ }^{1} \mathrm{H}$ NMR $\left(300 \mathrm{MHz} ; \mathrm{CDCl}_{3}\right) \delta 8.28-8.23(\mathrm{~m}, 1 \mathrm{H}), 7.58-7.38(\mathrm{~m}, 5 \mathrm{H}), 7.37-7.15$ (m, 5H), 7.04-6.95 (m, 1H), 5.15-5.05 (m, 1H), 4.50-4.37 (m, 1H), 3.85-3.68 (m, 1H), 3.25-3.14 (m, 1H), 2.76-2.66 (m, 2.5H), 2.60-2.48 (m, 0.5H), 2.10-1.96 (m, 1H), $1.76-1.54(\mathrm{~m}, 1 \mathrm{H}), 1.53-1.37(\mathrm{~m}, 3 \mathrm{H}), 1.47$ and $1.43(2 \mathrm{~s}, 9 \mathrm{H}), 1.28(\mathrm{t}, J=7.5 \mathrm{~Hz}, 3 \mathrm{H})$, 1.17-1.00 (m, 1H), 0.93-0.78 (m, 6H); MS (ESI) $\mathrm{m} / \mathrm{z} 542.6[\mathrm{M}+\mathrm{H}]^{+}$.

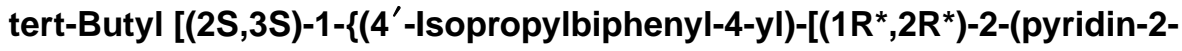
yl)cyclopropanecarbonyl]amino\}-3-methylpentan-2-yl]-carbamate (10d)-The procedure for 10a was followed using $68 \mathrm{mg}(0.13 \mathrm{mmol})$ of $9 \mathbf{a}$ and $34 \mathrm{mg}(0.2 \mathrm{mmol})$ of 4 isopropylphenylboronic acid to give $50 \mathrm{mg}(69 \%)$ of $10 \mathrm{~d}$ as a $1: 1$ diastereomeric mixture: ${ }^{1} \mathrm{H}$ NMR (300 MHz; $\left.\mathrm{CDCl}_{3}\right) \delta 8.26-8.20(\mathrm{~m}, 1 \mathrm{H}), 7.54-7.38(\mathrm{~m}, 5 \mathrm{H}), 7.35-7.12$ (m, 5H), 7.03-6.92 (m, 1H), 5.14-5.05 (m, 1H), 4.48-4.35 (m, 1H), 3.88-3.68 (m, 1H), 3.26-3.17 (m, 1H), 3.02-2.88 (m, 1H), 2.72-2.66 (m, 0.5H), 2.60-2.50 (m, 0.5H), 2.10-1.95 (m, 1H), 1.76-1.40 (m, 4H), 1.47 and $1.43(2 \mathrm{~s}, 9 \mathrm{H}), 1.29(\mathrm{~d}, J=6.0 \mathrm{~Hz}, 6 \mathrm{H})$, 1.18-1.02 (m, 1H), 0.95-0.82 (m, 6H); MS (ESI) $\mathrm{m} / \mathrm{z} 556.9[\mathrm{M}+\mathrm{H}]^{+}$.

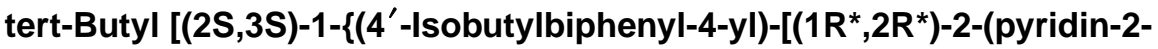
yl)cyclopropanecarbonyl]amino\}-3-methylpentan-2-yl]-carbamate (10e)-The procedure for 10a was followed using $30 \mathrm{mg}(0.058 \mathrm{mmol})$ of $9 \mathbf{a}$ and $16 \mathrm{mg}(0.087 \mathrm{mmol})$ of 4-isobutylphenylboronic acid to give $23 \mathrm{mg}(77 \%)$ of $10 \mathrm{e}$ as a 1:1 diastereomeric mixture: ${ }^{1} \mathrm{H}$ NMR (300 MHz; $\left.\mathrm{CDCl}_{3}\right) \delta 8.28-8.23(\mathrm{~m}, 1 \mathrm{H}), 7.60-7.38(\mathrm{~m}, 5 \mathrm{H}), 7.34-7.15$ (m, 5H), 7.04-6.95 (m, 1H), 5.14-5.05 (m, 1H), 4.50-4.36 (m, 1H), 3.86-3.68 (m, 1H), $3.24-3.14(\mathrm{~m}, 1 \mathrm{H}), 2.74-2.65(\mathrm{~m}, 0.5 \mathrm{H}), 2.60-2.46(\mathrm{~m}, 2.5 \mathrm{H}), 2.10-1.82(\mathrm{~m}, 2 \mathrm{H})$, $1.76-1.54(\mathrm{~m}, 1 \mathrm{H}), 1.53-1.36(\mathrm{~m}, 3 \mathrm{H}), 1.47$ and $1.43(2 \mathrm{~s}, 9 \mathrm{H}), 1.16-1.00(\mathrm{~m}, 1 \mathrm{H})$, $0.98-0.78(\mathrm{~m}, 12 \mathrm{H})$; MS (ESI) $\mathrm{m} / \mathrm{z} 570.6[\mathrm{M}+\mathrm{H}]^{+}$.

tert-Butyl [(2S,3S)-1-\{(4'-tert-butylbiphenyl-4-yl)-[(1 $\left.{ }^{\star}, 2 R^{\star}\right)$-2-(pyridin-2yl)cyclopropanecarbonyl]amino\}-3-methylpentan-2-yl]-carbamate (10f)-The procedure for 10a was followed using $30 \mathrm{mg}(0.058 \mathrm{mmol})$ of $9 \mathbf{a}$ and $16 \mathrm{mg}(0.087 \mathrm{mmol})$ of 4-tert-butylphenylboronic acid to give $20 \mathrm{mg}(61 \%)$ of $\mathbf{1 0 f}$ as a $1: 1$ diastereomeric mixture: ${ }^{1} \mathrm{H}$ NMR $\left(300 \mathrm{MHz} ; \mathrm{CDCl}_{3}\right) \delta 8.30-8.20(\mathrm{~m}, 1 \mathrm{H}), 7.58-7.45(\mathrm{~m}, 7 \mathrm{H}), 7.30-7.12$ (m, 3H), 7.00-6.92 (m, 1H), 5.12-5.04 (m, 1H), 4.45-4.33 (m, 1H), 3.82-3.66 (m, 1H), 3.25-3.15 (m, 1H), 2.72-2.65 (m, 0.5H), 2.60-2.48 (m, 0.5H), 2.08-1.95 (m, 1H), $1.75-1.40(\mathrm{~m}, 4 \mathrm{H}), 1.47$ and $1.43(2 \mathrm{~s}, 9 \mathrm{H}), 1.36(\mathrm{~s}, 9 \mathrm{H}), 1.16-1.02(\mathrm{~m}, 1 \mathrm{H}), 0.96-0.80(\mathrm{~m}$, $6 \mathrm{H}) ; \mathrm{MS}(\mathrm{ESI}) \mathrm{m} / \mathrm{z} 570.8[\mathrm{M}+\mathrm{H}]^{+}$.

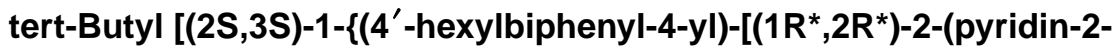
yl)cyclopropanecarbonyl]amino\}-3-methylpentan-2-yl]-carbamate (10g)-The procedure for 10a was followed using $68 \mathrm{mg}(0.13 \mathrm{mmol})$ of $9 \mathbf{a}$ and $42 \mathrm{mg}(0.2 \mathrm{mmol})$ of 4 hexylphenylboronic acid to give $40 \mathrm{mg}(51 \%)$ of $\mathbf{1 0 g}$ as a $1: 1$ diastereomeric mixture: ${ }^{1} \mathrm{H}$ NMR (300 MHz; $\left.\mathrm{CDCl}_{3}\right) \delta 8.28-8.21(\mathrm{~m}, 1 \mathrm{H}), 7.58-7.40(\mathrm{~m}, 5 \mathrm{H}), 7.36-7.15(\mathrm{~m}, 5 \mathrm{H})$, 
7.05-6.94 (m, 1H), 5.16-5.08 (m, 1H), 4.50-4.38 (m, 1H), 3.88-3.68 (m, 1H), 3.27-3.15 (m, 1H), 2.76-2.50 (m, 3H), 2.10-1.95 (m, 1H), 1.72-1.22 (m, 12H), 1.47 and $1.43(2 \mathrm{~s}$, 9H), 1.18-1.02 (m, 1H), 0.98-0.80 (m, 9H); MS (ESI) $\mathrm{m} / \mathrm{z} 599.0[\mathrm{M}+\mathrm{H}]^{+}$.

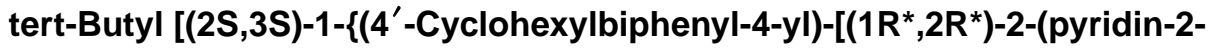
yl)cyclopropanecarbonyl]amino\}-3-methylpentan-2-yl]-carbamate (10h)-The procedure for 10a was followed using $30 \mathrm{mg}(0.058 \mathrm{mmol})$ of $9 \mathbf{a}$ and $18 \mathrm{mg}(0.087 \mathrm{mmol})$ of 4-cyclohexylphenylboronic acid to give $28 \mathrm{mg}(81 \%)$ of $\mathbf{1 0 h}$ as a $1: 1$ diastereomeric mixture: ${ }^{1} \mathrm{H}$ NMR (300 MHz; $\left.\mathrm{CDCl}_{3}\right) \delta 8.28-8.21(\mathrm{~m}, 1 \mathrm{H}), 7.58-7.40(\mathrm{~m}, 5 \mathrm{H}), 7.32-7.12$ (m, 5H), 7.02-6.92 (m, 1H), 5.15-5.05 (m, 1H), 4.50-4.36 (m, 1H), 3.88-3.68 (m, 1H), 3.26-3.13 (m, 1H), 2.75-2.64 (m, 0.5H), 2.60-2.48 (m, 1.5H), 2.05-1.71 (m, 7H), $1.69-1.25(\mathrm{~m}, 8 \mathrm{H}), 1.47$ and $1.43(2 \mathrm{~s}, 9 \mathrm{H}), 1.17-1.00(\mathrm{~m}, 1 \mathrm{H}), 0.96-0.80(\mathrm{~m}, 6 \mathrm{H}) ; \mathrm{MS}$ (ESI) $\mathrm{m} / \mathrm{z} 596.9[\mathrm{M}+\mathrm{H}]^{+}$.

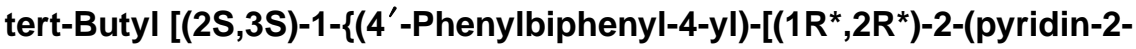
yl)cyclopropanecarbonyl]amino\}-3-methylpentan-2-yl]-carbamate (10i)-The procedure for 10a was followed using $30 \mathrm{mg}(0.058 \mathrm{mmol})$ of $9 \mathbf{a}$ and $18 \mathrm{mg}(0.087 \mathrm{mmol})$ of 4-biphenylboronic acid to give $22 \mathrm{mg}(65 \%)$ of $\mathbf{1 0 i}$ as a 1:1 diastereomeric mixture: ${ }^{1} \mathrm{H}$ NMR $\left(300 \mathrm{MHz} ; \mathrm{CDCl}_{3}\right) \delta 8.30-8.22(\mathrm{~m}, 1 \mathrm{H}), 7.70-7.42(\mathrm{~m}, 10 \mathrm{H}), 7.42-7.16(\mathrm{~m}, 5 \mathrm{H})$, 7.03-6.95 (m, 1H), 5.15-5.05 (m, 1H), 4.50-4.38 (m, 1H), 3.88-3.68 (m, 1H), 3.30-3.18 (m, 1H), 2.78-2.65 (m, 0.5H), 2.60-2.50 (m, 0.5H), 2.10-1.98 (m, 1H), 1.80-1.40 (m, 4H), 1.48 and $1.44(2 \mathrm{~s}, 9 \mathrm{H}), 1.20-1.02(\mathrm{~m}, 1 \mathrm{H}), 0.95-0.80(\mathrm{~m}, 6 \mathrm{H})$; MS (ESI) $\mathrm{m} / \mathrm{z} 590.4[\mathrm{M}+$ $\mathrm{H}]^{+}$.

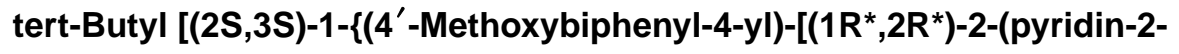
yl)cyclopropanecarbonyl]amino\}-3-methylpentan-2-yl]-carbamate (10j)-The procedure for 10a was followed using $68 \mathrm{mg}(0.13 \mathrm{mmol})$ of $\mathbf{9 a}$ and $30 \mathrm{mg}(0.2 \mathrm{mmol})$ of 4methoxyphenylboronic acid to give $52 \mathrm{mg}(74 \%)$ of $\mathbf{1 0 j}$ as a $1: 1$ diastereomeric mixture: ${ }^{1} \mathrm{H}$ NMR (300 MHz; $\left.\mathrm{CDCl}_{3}\right) \delta 8.30-8.25(\mathrm{~m}, 1 \mathrm{H}), 7.56-7.36(\mathrm{~m}, 5 \mathrm{H}), 7.32-7.16(\mathrm{~m}, 3 \mathrm{H})$, 7.04-6.93 (m, 3H), 5.15-5.06 (m, 1H), 4.50-4.37 (m, 1H), $3.85(\mathrm{~s}, 3 \mathrm{H}), 3.84-3.68(\mathrm{~m}, 1 \mathrm{H})$, 3.28-3.12 (m, 1H), 2.76-2.68 (m, 0.5H), 2.60-2.52 (m, 0.5H), 2.10-1.98 (m, 1H), $1.76-1.40(\mathrm{~m}, 4 \mathrm{H}), 1.47$ and $1.43(2 \mathrm{~s}, 9 \mathrm{H}), 1.20-1.02(\mathrm{~m}, 1 \mathrm{H}), 0.95-0.80(\mathrm{~m}, 6 \mathrm{H}) ; \mathrm{MS}$ (ESI) $\mathrm{m} / \mathrm{z} 544.7[\mathrm{M}+\mathrm{H}]^{+}$.

tert-Butyl [(2S,3S)-1-\{(4' -Trifluoromethoxybiphenyl-4-yl)-[(1 $\left.{ }^{\star}, 2 \mathrm{R}^{\star}\right)-2-$ (pyridin-2-yl)cyclopropanecarbonyl]amino\}-3-methylpentan-2-yl]carbamate (10k)-The procedure for 10a was followed using $30 \mathrm{mg}(0.058 \mathrm{mmol})$ of $\mathbf{9 a}$ and $18 \mathrm{mg}$ (0.087 mmol) of 4-trifluoromethoxyphenylboronic acid to give $18 \mathrm{mg}(52 \%)$ of $10 \mathrm{k}$ as a 1:1 diastereomeric mixture: ${ }^{1} \mathrm{H}$ NMR $\left(300 \mathrm{MHz} ; \mathrm{CDCl}_{3}\right) \delta 8.30-8.22(\mathrm{~m}, 1 \mathrm{H}), 7.58-7.40(\mathrm{~m}$, $5 \mathrm{H}), 7.38-7.16(\mathrm{~m}, 5 \mathrm{H}), 7.04-6.96(\mathrm{~m}, 1 \mathrm{H}), 5.10-5.03(\mathrm{~m}, 1 \mathrm{H}), 4.50-4.38(\mathrm{~m}, 1 \mathrm{H})$, $3.82-3.68(\mathrm{~m}, 1 \mathrm{H}), 3.28-3.16(\mathrm{~m}, 1 \mathrm{H}), 2.76-2.68(\mathrm{~m}, 0.5 \mathrm{H}), 2.60-2.50(\mathrm{~m}, 0.5 \mathrm{H})$, 2.08-1.94 (m, 1H), 1.75-1.40 (m, 4H), 1.47 and $1.43(2 \mathrm{~s}, 9 \mathrm{H}), 1.20-1.05(\mathrm{~m}, 1 \mathrm{H})$, 0.96-0.82 (m, 6H); MS (ESI) $\mathrm{m} / z 598.9[\mathrm{M}+\mathrm{H}]^{+}$. 


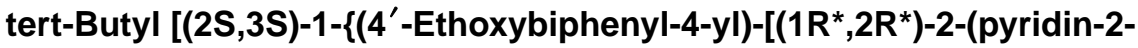
yl)cyclopropanecarbonyl]amino\}-3-methylpentan-2-yl]-carbamate (10I)—The procedure for 10a was followed using $30 \mathrm{mg}(0.058 \mathrm{mmol})$ of $9 \mathrm{a}$ and $14 \mathrm{mg}(0.087 \mathrm{mmol})$ of 4-ethoxyphenylboronic acid to give $22 \mathrm{mg}(68 \%)$ of $\mathbf{1 0 1}$ as a 1:1 diastereomeric mixture: ${ }^{1} \mathrm{H}$ NMR (300 MHz; $\left.\mathrm{CDCl}_{3}\right) \delta 8.28-8.20(\mathrm{~m}, 1 \mathrm{H}), 7.55-7.40(\mathrm{~m}, 5 \mathrm{H}), 7.30-7.12$ (m, 3H), 7.02-6.92 (m, 3H), 5.12-5.05 (m, 1H), 4.48-4.38 (m, 1H), 4.15-4.04 (m, 2H), 3.86-3.68 (m, 1H), 3.26-3.15 (m, 1H), 2.76-2.68 (m, 0.5H), 2.60-2.52 (m, $0.5 \mathrm{H})$, 2.08-1.96 (m, 1H), 1.78-1.40 (m, 4H), 1.47 and $1.42(2 \mathrm{~s}, 9 \mathrm{H}), 1.20-1.04(\mathrm{~m}, 1 \mathrm{H})$, $0.94-0.82(\mathrm{~m}, 9 \mathrm{H}) ; \mathrm{MS}$ (ESI) $\mathrm{m} / \mathrm{z} 558.8[\mathrm{M}+\mathrm{H}]^{+}$.

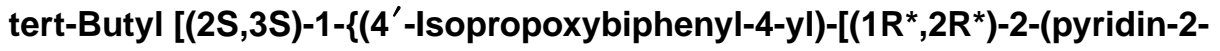
yl)cyclopropanecarbonyl]amino\}-3-methylpentan-2-yl]-carbamate (10m)-The procedure for 10a was followed using $30 \mathrm{mg}(0.058 \mathrm{mmol})$ of $9 \mathbf{a}$ and $16 \mathrm{mg}(0.087 \mathrm{mmol})$ of 4-isopropoxyphenylboronic acid to give $20 \mathrm{mg}(60 \%)$ of $\mathbf{1 0 m}$ as a $1: 1$ diastereomeric mixture: ${ }^{1} \mathrm{H}$ NMR (300 MHz; $\left.\mathrm{CDCl}_{3}\right) \delta 8.28-8.22(\mathrm{~m}, 1 \mathrm{H}), 7.54-7.38(\mathrm{~m}, 5 \mathrm{H}), 7.32-7.15$ (m, 3H), 7.05-6.92 (m, 3H), 5.15-5.06 (m, 1H), 4.68-4.55 (m, 1H), 4.50-4.38 (m, 1H), $3.86-3.68(\mathrm{~m}, 1 \mathrm{H}), 3.25-3.15(\mathrm{~m}, 1 \mathrm{H}), 2.76-2.68(\mathrm{~m}, 0.5 \mathrm{H}), 2.60-2.50(\mathrm{~m}, 0.5 \mathrm{H})$, 2.10-1.96 (m, 1H), 1.77-1.40 (m, 4H), 1.47 and $1.43(2 \mathrm{~s}, 9 \mathrm{H}), 1.37(\mathrm{~d}, J=6.0 \mathrm{~Hz}, 6 \mathrm{H})$, 1.20-1.05 (m, 1H), 0.95-0.80 (m, 6H); MS (ESI) $\mathrm{m} / \mathrm{z} 572.9[\mathrm{M}+\mathrm{H}]^{+}$.

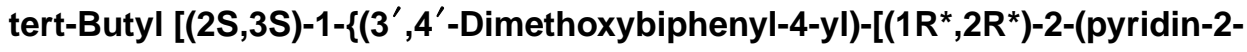
yl)cyclopropanecarbonyl]amino\}-3-methylpentan-2-yl]carbamate (10n)-The procedure for 10a was followed using $30 \mathrm{mg}(0.058 \mathrm{mmol})$ of $9 \mathbf{a}$ and $16 \mathrm{mg}(0.087 \mathrm{mmol})$ of 3,4-dimethoxyphenylboronic acid to give $19 \mathrm{mg}(57 \%)$ of $10 \mathrm{n}$ as a $1: 1$ diastereomeric mixture: ${ }^{1} \mathrm{H}$ NMR $\left(300 \mathrm{MHz} ; \mathrm{CDCl}_{3}\right) \delta 8.28-8.20(\mathrm{~m}, 1 \mathrm{H}), 7.55-7.40(\mathrm{~m}, 2 \mathrm{H}), 7.32-6.90$ $(\mathrm{m}, 8 \mathrm{H}), 5.15-5.08(\mathrm{~m}, 1 \mathrm{H}), 4.50-4.38(\mathrm{~m}, 1 \mathrm{H}), 3.96(\mathrm{~s}, 3 \mathrm{H}), 3.33(\mathrm{~s}, 3 \mathrm{H}), 3.85-3.68(\mathrm{~m}$, $1 \mathrm{H}), 3.26-3.15(\mathrm{~m}, 1 \mathrm{H}), 2.78-2.68(\mathrm{~m}, 0.5 \mathrm{H}), 2.62-2.53(\mathrm{~m}, 0.5 \mathrm{H}), 2.10-1.98(\mathrm{~m}, 1 \mathrm{H})$, $1.76-1.40(\mathrm{~m}, 4 \mathrm{H}), 1.47$ and $1.43(2 \mathrm{~s}, 9 \mathrm{H}), 1.20-1.03(\mathrm{~m}, 1 \mathrm{H}), 0.96-0.80(\mathrm{~m}, 6 \mathrm{H}) ; \mathrm{MS}$ (ESI) $\mathrm{m} / \mathrm{z} 574.9[\mathrm{M}+\mathrm{H}]^{+}$.

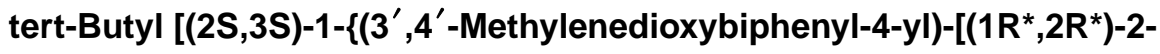
(pyridin-2-yl)cyclopropanecarbonyl]amino\}-3-methylpentan-2-yl]carbamate (100)-The procedure for 10a was followed using $30 \mathrm{mg}(0.058 \mathrm{mmol})$ of $9 \mathbf{a}$ and $14 \mathrm{mg}$ ( $0.087 \mathrm{mmol})$ of 3,4-methylenedioxyphenylboronic acid to give $20 \mathrm{mg}(62 \%)$ of $\mathbf{1 0 0}$ as a $1: 1$ diastereomeric mixture: ${ }^{1} \mathrm{H}$ NMR $\left(300 \mathrm{MHz} ; \mathrm{CDCl}_{3}\right) \delta 8.30-8.22(\mathrm{~m}, 1 \mathrm{H}), 7.56-7.33(\mathrm{~m}$, $3 \mathrm{H}), 7.32-7.15(\mathrm{~m}, 3 \mathrm{H}), 7.08-6.92(\mathrm{~m}, 3 \mathrm{H}), 6.90-6.85(\mathrm{~m}, 1 \mathrm{H}), 6.01(\mathrm{~s}, 2 \mathrm{H}), 5.10-5.04(\mathrm{~m}$, $1 \mathrm{H}), 4.50-4.38(\mathrm{~m}, 1 \mathrm{H}), 3.85-3.68(\mathrm{~m}, 1 \mathrm{H}), 3.26-3.16(\mathrm{~m}, 1 \mathrm{H}), 2.76-2.66(\mathrm{~m}, 0.5 \mathrm{H})$, 2.60-2.50 (m, 0.5H), 2.10-1.98 (m, 1H), 1.76-1.40 (m, 4H), 1.47 and $1.42(2 \mathrm{~s}, 9 \mathrm{H})$, 1.20-1.02 (m, 1H), 0.96-0.80 (m, 6H); MS (ESI) $\mathrm{m} / z 558.7[\mathrm{M}+\mathrm{H}]^{+}$.

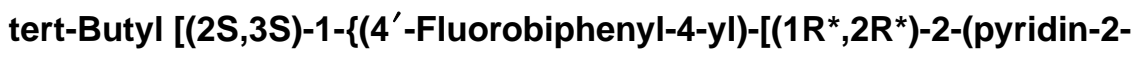
yl)cyclopropanecarbonyl]amino\}-3-methylpentan-2-yl]-carbamate (10p)-The procedure for 10a was followed using $30 \mathrm{mg}(0.058 \mathrm{mmol})$ of $9 \mathbf{a}$ and $12 \mathrm{mg}(0.087 \mathrm{mmol})$ of 4-fluorophenylboronic acid to give $20 \mathrm{mg}$ (74\%) of 10p as a 1:1 diastereomeric mixture: ${ }^{1} \mathrm{H}$ NMR $\left(300 \mathrm{MHz} ; \mathrm{CDCl}_{3}\right) \delta 8.30-8.22(\mathrm{~m}, 1 \mathrm{H}), 7.56-7.48(\mathrm{~m}, 4 \mathrm{H}), 7.30-7.18$ 
(m, 6H), 7.02-6.92 (m, 1H), 5.12-5.05 (m, 1H), 4.52-4.38 (m, 1H), 3.82-3.65 (m, 1H), 3.25-3.12 (m, 1H), 2.76-2.65 (m, 0.5H), 2.61-2.48 (m, 0.5H), 2.08-1.95 (m, 1H), $1.78-1.55(\mathrm{~m}, 1 \mathrm{H}), 1.54-1.37(\mathrm{~m}, 3 \mathrm{H}), 1.47$ and $1.43(2 \mathrm{~s}, 9 \mathrm{H}), 1.18-1.00(\mathrm{~m}, 1 \mathrm{H})$, $0.95-0.80(\mathrm{~m}, 6 \mathrm{H}) ; \mathrm{MS}(\mathrm{ESI}) \mathrm{m} / \mathrm{z} 532.5[\mathrm{M}+\mathrm{H}]^{+}$.

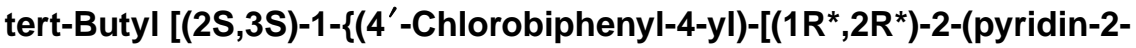
yl)cyclopropanecarbonyl]amino\}-3-methylpentan-2-yl]-carbamate (10q)-The procedure for 10a was followed using $30 \mathrm{mg}(0.058 \mathrm{mmol})$ of $9 \mathbf{a}$ and $14 \mathrm{mg}(0.087 \mathrm{mmol})$ of 4-chlorophenylboronic acid to give $22 \mathrm{mg}$ (74\%) of $\mathbf{1 0 q}$ as a 1:1 diastereomeric mixture: ${ }^{1} \mathrm{H}$ NMR $\left(300 \mathrm{MHz} ; \mathrm{CDCl}_{3}\right) \delta 8.30-8.21(\mathrm{~m}, 1 \mathrm{H}), 7.55-7.36(\mathrm{~m}, 6 \mathrm{H}), 7.30-7.15$ $(\mathrm{m}, 4 \mathrm{H}), 7.02-6.95(\mathrm{~m}, 1 \mathrm{H}), 5.10-5.00(\mathrm{~m}, 1 \mathrm{H}), 4.50-4.35(\mathrm{~m}, 1 \mathrm{H}), 3.80-3.62(\mathrm{~m}, 1 \mathrm{H})$, 3.26-3.15 (m, 1H), 2.76-2.65 (m, 0.5H), 2.61-2.48 (m, 0.5H), 2.08-1.95 (m, 1H), $1.75-1.55(\mathrm{~m}, 1 \mathrm{H}), 1.54-1.37(\mathrm{~m}, 3 \mathrm{H}), 1.47$ and $1.42(2 \mathrm{~s}, 9 \mathrm{H}), 1.16-1.00(\mathrm{~m}, 1 \mathrm{H})$, $0.95-0.80(\mathrm{~m}, 6 \mathrm{H})$; MS (ESI) $\mathrm{m} / \mathrm{z} 548.5[\mathrm{M}+\mathrm{H}]^{+}$.

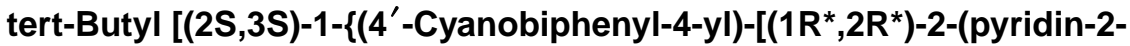
yl)cyclopropanecarbonyl]amino\}-3-methylpentan-2-yl]-carbamate (10r)-The procedure for 10a was followed using $30 \mathrm{mg}(0.058 \mathrm{mmol})$ of $9 \mathrm{a}$ and $13 \mathrm{mg}(0.087 \mathrm{mmol})$ of 4-cyanophenylboronic acid to give $20 \mathrm{mg}(64 \%)$ of $\mathbf{1 0 r}$ as a $1: 1$ diastereomeric mixture: ${ }^{1} \mathrm{H}$ NMR (300 MHz; $\left.\mathrm{CDCl}_{3}\right) \delta 8.28-8.20(\mathrm{~m}, 1 \mathrm{H}), 7.76-7.42(\mathrm{~m}, 7 \mathrm{H}), 7.40-7.16$ $(\mathrm{m}, 3 \mathrm{H}), 7.02-6.92(\mathrm{~m}, 1 \mathrm{H}), 5.10-5.00(\mathrm{~m}, 1 \mathrm{H}), 4.50-4.38(\mathrm{~m}, 1 \mathrm{H}), 3.80-3.64(\mathrm{~m}, 1 \mathrm{H})$, 3.26-3.14 (m, 1H), 2.76-2.66 (m, 0.5H), 2.61-2.52 (m, 0.5H), 2.08-1.95 (m, 1H), $1.75-1.40(\mathrm{~m}, 4 \mathrm{H}), 1.47$ and $1.42(2 \mathrm{~s}, 9 \mathrm{H}), 1.18-1.00(\mathrm{~m}, 1 \mathrm{H}), 0.96-0.80(\mathrm{~m}, 6 \mathrm{H}) ; \mathrm{MS}$ (ESI) $\mathrm{m} / \mathrm{z} 539.3[\mathrm{M}+\mathrm{H}]^{+}$.

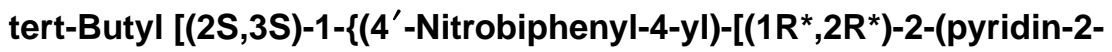
yl)cyclopropanecarbonyl]amino\}-3-methylpentan-2-yl]-carbamate (10s)—The procedure for 10a was followed using $30 \mathrm{mg}(0.058 \mathrm{mmol})$ of $9 \mathbf{a}$ and $15 \mathrm{mg}(0.087 \mathrm{mmol})$ of 4-nitrophenylboronic acid to give $21 \mathrm{mg}(65 \%)$ of $10 \mathrm{~s}$ as a $1: 1$ diastereomeric mixture: ${ }^{1} \mathrm{H}$ NMR (300 MHz; $\left.\mathrm{CDCl}_{3}\right) \delta 8.35-8.21(\mathrm{~m}, 3 \mathrm{H}), 7.75-7.44(\mathrm{~m}, 5 \mathrm{H}), 7.40-7.16(\mathrm{~m}, 3 \mathrm{H})$, 7.02-6.95 (m, 1H), 5.08-4.98 (m, 1H), 4.50-4.38 (m, 1H), 3.80-3.62 (m, 1H), 3.28-3.18 (m, 1H), 2.76-2.66 (m, 0.5H), 2.60-2.50 (m, 0.5H), 2.08-1.94 (m, 1H), 1.76-1.40 (m, 4H), 1.47 and $1.42(2 \mathrm{~s}, 9 \mathrm{H}), 1.17-1.00(\mathrm{~m}, 1 \mathrm{H}), 0.95-0.80(\mathrm{~m}, 6 \mathrm{H})$; MS (ESI) $\mathrm{m} / z 559.4[\mathrm{M}+$ $\mathrm{H}]^{+}$.

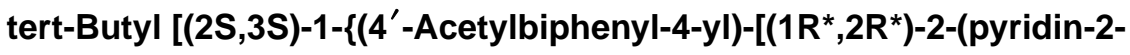
yl)cyclopropanecarbonyl]amino\}-3-methylpentan-2-yl]-carbamate (10t)—The procedure for 10a was followed using $30 \mathrm{mg}(0.058 \mathrm{mmol})$ of $9 \mathrm{a}$ and $14 \mathrm{mg}(0.087 \mathrm{mmol})$ of 4-acetylphenylboronic acid to give $25 \mathrm{mg}(76 \%)$ of $\mathbf{1 0 t}$ as a $1: 1$ diastereomeric mixture: ${ }^{1} \mathrm{H}$ NMR (300 MHz; $\left.\mathrm{CDCl}_{3}\right) \delta 8.30-8.21(\mathrm{~m}, 1 \mathrm{H}), 8.06-7.98(\mathrm{~m}, 2 \mathrm{H}), 7.68-7.48$ $(\mathrm{m}, 5 \mathrm{H}), 7.38-7.15(\mathrm{~m}, 3 \mathrm{H}), 7.02-6.93(\mathrm{~m}, 1 \mathrm{H}), 5.10-5.00(\mathrm{~m}, 1 \mathrm{H}), 4.50-4.36(\mathrm{~m}, 1 \mathrm{H})$, $3.80-3.62(\mathrm{~m}, 1 \mathrm{H}), 3.28-3.15(\mathrm{~m}, 1 \mathrm{H}), 2.75-2.65(\mathrm{~m}, 0.5 \mathrm{H}), 2.64(\mathrm{~s}, 3 \mathrm{H}), 2.61-2.48(\mathrm{~m}$, $0.5 \mathrm{H}), 2.08-1.95(\mathrm{~m}, 1 \mathrm{H}), 1.76-1.55(\mathrm{~m}, 1 \mathrm{H}), 1.54-1.37(\mathrm{~m}, 3 \mathrm{H}), 1.47$ and $1.43(2 \mathrm{~s}, 9 \mathrm{H})$, 1.16-0.98 (m, 1H), 0.92-0.78 (m, 6H); MS (ESI) $\mathrm{m} / z 557.2[\mathrm{M}+\mathrm{H}]^{+}$. 


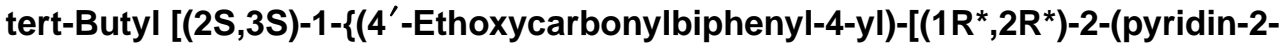
yl)cyclopropanecarbonyl]amino\}-3-methylpentan-2-yl]carbamate (10u)—The procedure for 10a was followed using $30 \mathrm{mg}(0.058 \mathrm{mmol})$ of $9 \mathrm{a}$ and $17 \mathrm{mg}(0.087 \mathrm{mmol})$ of 4-ethoxycarbonylphenylboronic acid to give $22 \mathrm{mg}(65 \%)$ of $10 \mathbf{u}$ as a 1:1 diastereomeric mixture: ${ }^{1} \mathrm{H}$ NMR $\left(300 \mathrm{MHz} ; \mathrm{CDCl}_{3}\right) \delta 8.30-8.22(\mathrm{~m}, 1 \mathrm{H}), 8.16-8.08(\mathrm{~m}, 2 \mathrm{H}), 7.66-7.48$ (m, 5H), 7.38-7.16 (m, 3H), 7.02-6.95 (m, 1H), 5.10-5.02 (m, 1H), 4.50-4.36 (m, 3H), $3.85-3.66(\mathrm{~m}, 1 \mathrm{H}), 3.28-3.16(\mathrm{~m}, 1 \mathrm{H}), 2.75-2.65(\mathrm{~m}, 0.5 \mathrm{H}), 2.62-2.50(\mathrm{~m}, 0.5 \mathrm{H})$, 2.10-1.98 (m, 1H), 1.76-1.37 (m, 4H), 1.47 and $1.43(2 \mathrm{~s}, 9 \mathrm{H}), 1.20-1.02(\mathrm{~m}, 1 \mathrm{H})$, $0.95-0.81(\mathrm{~m}, 9 \mathrm{H}) ; \mathrm{MS}$ (ESI) $\mathrm{m} / \mathrm{z} 586.5[\mathrm{M}+\mathrm{H}]^{+}$.

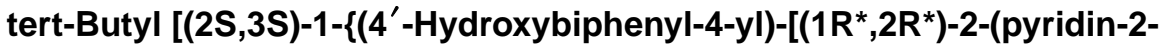
yl)cyclopropanecarbonyl]amino\}-3-methylpentan-2-yl]-carbamate (10v)-The procedure for 10a was followed using $30 \mathrm{mg}(0.058 \mathrm{mmol})$ of $9 \mathbf{a}$ and $25 \mathrm{mg}(0.087 \mathrm{mmol})$ of 4-(tert-butoxycarboxy)phenylboronic acid to give $22 \mathrm{mg}(72 \%)$ of $10 \mathrm{v}$ as a $1: 1$ diastereomeric mixture: ${ }^{1} \mathrm{H}$ NMR $\left(300 \mathrm{MHz} ; \mathrm{CDCl}_{3}\right) \delta 8.52(\mathrm{br} \mathrm{s}, 1 \mathrm{H}), 8.28-8.18(\mathrm{~m}, 1 \mathrm{H})$, 7.60-7.50 (m, 1H), 7.38-7.10 (m, 7H), 7.08-7.00 (m, 1H), 7.76-6.68 (m, 2H), 5.26-5.18 (m, $1 \mathrm{H}), 4.52-4.40(\mathrm{~m}, 1 \mathrm{H}), 3.82-3.68(\mathrm{~m}, 1 \mathrm{H}), 3.20-3.08(\mathrm{~m}, 1 \mathrm{H}), 2.78-2.68(\mathrm{~m}, 0.5 \mathrm{H})$, 2.62-2.52 (m, 0.5H), 2.15-2.08 (m, 1H), 1.76-1.40 (m, 4H), 1.51 and $1.46(2 \mathrm{~s}, 9 \mathrm{H})$, 1.20-1.02 (m, 1H), 0.96-0.80 (m, 6H); MS (ESI) $\mathrm{m} / \mathrm{z} 530.9[\mathrm{M}+\mathrm{H}]^{+}$.

tert-Butyl [(2S,3S)-1-\{[4' -(tert-Butoxycarbonylamino)biphenyl-4-yl]-[(1R ${ }^{*}$, 2R*)-2-(pyridin-2-yl)cyclopropanecarbonyl]amino\}-3-methylpentan-2-

yl]carbamate (10w)-The procedure for 10a was followed using $30 \mathrm{mg}(0.058 \mathrm{mmol})$ of 9a and $21 \mathrm{mg}(0.087 \mathrm{mmol})$ of 4-(tert-butoxycarbonylamino)phenylboronic acid to give 22 $\mathrm{mg}(60 \%)$ of $10 \mathrm{w}$ as a $1: 1$ diastereomeric mixture: ${ }^{1} \mathrm{H} \mathrm{NMR}\left(300 \mathrm{MHz} ; \mathrm{CDCl}_{3}\right) \delta 8.28-8.20$ (m, 1H), 7.52-7.40 (m, 7H), 7.30-7.12 (m, 3H), 7.02-6.94 (m, 1H), $6.68(\mathrm{~s}, 1 \mathrm{H}), 5.12-5.06$ (m, 1H), 4.48-4.38 (m, 1H), 3.82-3.67 (m, 1H), 3.25-3.15 (m, 1H), 2.76-2.68 (m, 0.5H), $2.58-2.48(\mathrm{~m}, 0.5 \mathrm{H}), 2.08-1.98(\mathrm{~m}, 1 \mathrm{H}), 1.77-1.40(\mathrm{~m}, 4 \mathrm{H}), 1.53(\mathrm{~s}, 9 \mathrm{H}), 1.47$ and $1.43(2 \mathrm{~s}$, 9H), 1.20-1.02 (m, 1H), 0.95-0.80 (m, 6H); MS (ESI) $\mathrm{m} / \mathrm{z} 629.8[\mathrm{M}+\mathrm{H}]^{+}$.

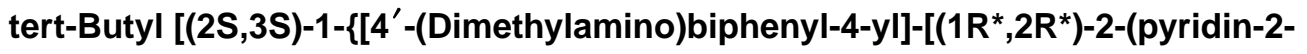
yl)cyclopropanecarbonyl]amino\}-3-methylpentan-2-yl]carbamate (10x)-The procedure for 10a was followed using $68 \mathrm{mg}(0.13 \mathrm{mmol})$ of $9 \mathbf{a}$ and $35 \mathrm{mg}(0.2 \mathrm{mmol})$ of 4(dimethylamino)phenylboronic acid to give $52 \mathrm{mg}(72 \%)$ of $\mathbf{1 0 x}$ as a 1:1 diastereomeric mixture: ${ }^{1} \mathrm{H}$ NMR (300 MHz; $\left.\mathrm{CDCl}_{3}\right) \delta 8.30-8.22(\mathrm{~m}, 1 \mathrm{H}), 7.55-7.36(\mathrm{~m}, 5 \mathrm{H}), 7.30-7.12$ $(\mathrm{m}, 3 \mathrm{H}), 7.02-6.96(\mathrm{~m}, 1 \mathrm{H}), 6.84-6.76(\mathrm{~m}, 2 \mathrm{H}), 5.18-5.10(\mathrm{~m}, 1 \mathrm{H}), 4.50-4.38(\mathrm{~m}, 1 \mathrm{H})$, 3.88-3.70 (m, 1H), 3.26-3.16 (m, 1H), $2.99(\mathrm{~s}, 6 \mathrm{H}), 2.76-2.68(\mathrm{~m}, 0.5 \mathrm{H}), 2.60-2.50(\mathrm{~m}$, $0.5 \mathrm{H}), 2.10-1.98(\mathrm{~m}, 1 \mathrm{H}), 1.72-1.40(\mathrm{~m}, 4 \mathrm{H}), 1.47$ and $1.43(2 \mathrm{~s}, 9 \mathrm{H}), 1.20-1.02(\mathrm{~m}, 1 \mathrm{H})$, $0.98-0.82(\mathrm{~m}, 6 \mathrm{H})$; MS (ESI) $\mathrm{m} / \mathrm{z} 557.8[\mathrm{M}+\mathrm{H}]^{+}$.

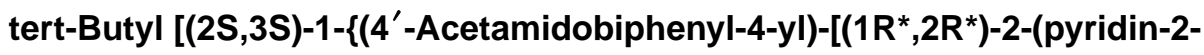
yl)cyclopropanecarbonyl]amino\}-3-methylpentan-2-yl]-carbamate (10y)-The procedure for 10a was followed using $30 \mathrm{mg}(0.058 \mathrm{mmol})$ of $9 \mathbf{a}$ and $15.6 \mathrm{mg}(0.087 \mathrm{mmol})$ of 4-acetamidophenylboronic acid to give $18 \mathrm{mg}(54 \%)$ of $\mathbf{1 0 y}$ as a $1: 1$ diastereomeric mixture: ${ }^{1} \mathrm{H}$ NMR $\left(300 \mathrm{MHz} ; \mathrm{CDCl}_{3}\right) \delta 8.28-8.20(\mathrm{~m}, 1 \mathrm{H}), 7.96-7.80(\mathrm{~m}, 1 \mathrm{H}), 7.62-7.34$ 
(m, 7H), 7.30-7.15 (m, 3H), 7.02-6.96 (m, 1H), 5.18-5.08 (m, 1H), 4.50-4.38 (m, 1H), 3.82-3.66 (m, 1H), 3.28-3.15 (m, 1H), 2.76-2.67 (m, 0.5H), 2.60-2.50 (m, 0.5H), $2.18(\mathrm{~s}$, $3 \mathrm{H}), 2.08-1.95(\mathrm{~m}, 1 \mathrm{H}), 1.76-1.40(\mathrm{~m}, 4 \mathrm{H}), 1.47$ and $1.43(2 \mathrm{~s}, 9 \mathrm{H}), 1.20-1.02(\mathrm{~m}, 1 \mathrm{H})$, $0.96-0.80(\mathrm{~m}, 6 \mathrm{H})$; MS (ESI) $\mathrm{m} / \mathrm{z} 571.6[\mathrm{M}+\mathrm{H}]^{+}$.

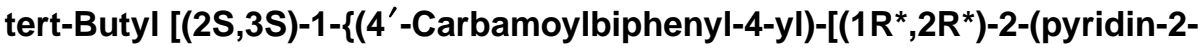
yl)cyclopropanecarbonyl]amino\}-3-methylpentan-2-yl]-carbamate (10z)-The procedure for 10a was followed using $30 \mathrm{mg}(0.058 \mathrm{mmol})$ of $9 \mathrm{a}$ and $14 \mathrm{mg}(0.087 \mathrm{mmol})$ of 4-carbamoylphenylboronic acid to give $18 \mathrm{mg}(56 \%)$ of $10 \mathrm{z}$ as a $1: 1$ diastereomeric mixture: ${ }^{1} \mathrm{H}$ NMR $\left(300 \mathrm{MHz} ; \mathrm{CDCl}_{3}\right) \delta 8.30-8.21(\mathrm{~m}, 1 \mathrm{H}), 7.92-7.86(\mathrm{~m}, 2 \mathrm{H}), 7.66-7.46$ (m, 5H), 7.40-7.18 (m, 3H), 7.05-6.98 (m, 1H), 6.16 (br s, 1H), 5.80 (br s, 1H), 5.10-5.05 $(\mathrm{m}, 1 \mathrm{H}), 4.50-4.38(\mathrm{~m}, 1 \mathrm{H}), 3.82-3.64(\mathrm{~m}, 1 \mathrm{H}), 3.28-3.16(\mathrm{~m}, 1 \mathrm{H}), 2.76-2.68(\mathrm{~m}, 0.5 \mathrm{H})$, 2.60-2.50 (m, 0.5H), 2.08-1.96 (m, 1H), 1.76-1.40 (m, 4H), 1.47 and $1.42(2 \mathrm{~s}, 9 \mathrm{H})$, 1.20-1.02 (m, 1H), 0.95-0.81 (m, 6H); MS (ESI) $\mathrm{m} / \mathrm{z} 558.1[\mathrm{M}+\mathrm{H}]^{+}$.

tert-Butyl [(2S,3S)-1-\{[4' -(Dimethylcarbamoyl)biphenyl-4-yl]-[(1 $\left.\mathbf{R}^{\star}, 2 \mathrm{R}^{\star}\right)$-2(pyridin-2-yl)cyclopropanecarbonyl]amino\}-3-methylpentan-2-yl]carbamate (10aa)-The procedure for 10a was followed using $30 \mathrm{mg}(0.058 \mathrm{mmol})$ of $\mathbf{9 a}$ and $17 \mathrm{mg}$ ( $0.087 \mathrm{mmol}$ ) of 4-(dimethylcarbamoyl) phenylboronic acid to give $20 \mathrm{mg}(56 \%)$ of 10aa as a 1:1 diastereomeric mixture: ${ }^{1} \mathrm{H}$ NMR $\left(300 \mathrm{MHz} ; \mathrm{CDCl}_{3}\right) \delta 8.28-8.22(\mathrm{~m}, 1 \mathrm{H}), 7.60-7.38$ $(\mathrm{m}, 7 \mathrm{H}), 7.32-7.12(\mathrm{~m}, 3 \mathrm{H}), 7.02-6.95(\mathrm{~m}, 1 \mathrm{H}), 5.10-5.00(\mathrm{~m}, 1 \mathrm{H}), 4.50-4.38(\mathrm{~m}, 1 \mathrm{H})$, 3.82-3.65 (m, 1H), 3.28-3.16 (m, 1H), $3.14(\mathrm{~s}, 3 \mathrm{H}), 3.04(\mathrm{~s}, 3 \mathrm{H}), 2.75-2.66(\mathrm{~m}, 0.5 \mathrm{H})$, $2.60-2.50(\mathrm{~m}, 0.5 \mathrm{H}), 2.08-1.96(\mathrm{~m}, 1 \mathrm{H}), 1.76-1.40(\mathrm{~m}, 4 \mathrm{H}), 1.47$ and $1.43(2 \mathrm{~s}, 9 \mathrm{H})$, 1.20-1.02 (m, 1H), 0.96-0.80 (m, 6H); MS (ESI) $\mathrm{m} / \mathrm{z} 585.8[\mathrm{M}+\mathrm{H}]^{+}$.

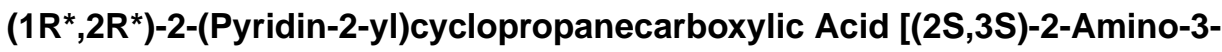
methylpentyl]-(4' -methylbiphenyl-4-yl)amide (5a)-The procedure for $4 \mathbf{a}$ was followed using $20 \mathrm{mg}(0.038 \mathrm{mmol})$ of $10 \mathrm{a}$ and $1 \mathrm{~mL}$ of $4 \mathrm{M} \mathrm{HCl}$ in dioxane to give $18 \mathrm{mg}$ (95\%) of 5a dihydrochloride as a 1:1 diastereomeric mixture: ${ }^{1} \mathrm{H}$ NMR $\left(300 \mathrm{MHz} ; \mathrm{CD}_{3} \mathrm{OD}\right)$ $\delta 8.68-8.55(\mathrm{~m}, 1 \mathrm{H}), 8.42-8.30(\mathrm{~m}, 1 \mathrm{H}), 7.88-7.40(\mathrm{~m}, 8 \mathrm{H}), 7.30-7.20(\mathrm{~m}, 2 \mathrm{H}), 4.50-4.40$ $(\mathrm{m}, 0.5 \mathrm{H}), 4.40-4.28(\mathrm{~m}, 0.5 \mathrm{H}), 3.88-3.56(\mathrm{~m}, 1 \mathrm{H}), 3.48-3.38(\mathrm{~m}, 1 \mathrm{H}), 3.12-3.05(\mathrm{~m}$, $0.5 \mathrm{H}), 3.05-2.96(\mathrm{~m}, 0.5 \mathrm{H}), 2.37(\mathrm{~s}, 3 \mathrm{H}), 2.22-2.10(\mathrm{~m}, 1 \mathrm{H}), 2.10-1.88(\mathrm{~m}, 1 \mathrm{H}), 1.87-1.60$ $(\mathrm{m}, 2 \mathrm{H}), 1.48-1.15(\mathrm{~m}, 2 \mathrm{H}), 1.06-0.80(\mathrm{~m}, 6 \mathrm{H})$; HRMS (ESI) calcd for $\mathrm{C}_{28} \mathrm{H}_{33} \mathrm{~N}_{3} \mathrm{O}[\mathrm{M}+$ $\mathrm{H}]^{+}:$428.2696. Found: 428.2701.

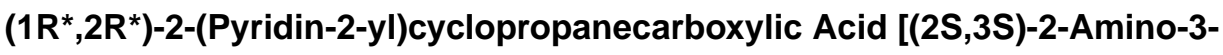
methylpentyl]-(4' -trifluoromethylbiphenyl-4-yl)amide (5b)-The procedure for $\mathbf{4 a}$ was followed using $20 \mathrm{mg}(0.034 \mathrm{mmol})$ of $\mathbf{1 0 b}$ and $1 \mathrm{~mL}$ of $4 \mathrm{M} \mathrm{HCl}$ in dioxane to give 18 $\mathrm{mg}(95 \%)$ of $\mathbf{5 b}$ dihydrochloride as a 1:1 diastereomeric mixture: ${ }^{1} \mathrm{H}$ NMR (300 MHz; $\left.\mathrm{CD}_{3} \mathrm{OD}\right) \delta 8.70-8.58(\mathrm{~m}, 1 \mathrm{H}), 8.42-8.30(\mathrm{~m}, 1 \mathrm{H}), 7.90-7.50(\mathrm{~m}, 10 \mathrm{H}), 4.55-4.40(\mathrm{~m}$, $0.5 \mathrm{H}), 4.40-4.26(\mathrm{~m}, 0.5 \mathrm{H}), 3.90-3.60(\mathrm{~m}, 1 \mathrm{H}), 3.50-3.38(\mathrm{~m}, 1 \mathrm{H}), 3.15-3.05(\mathrm{~m}, 0.5 \mathrm{H})$, $3.05-2.92(\mathrm{~m}, 0.5 \mathrm{H}), 2.25-2.10(\mathrm{~m}, 1 \mathrm{H}), 2.10-1.92(\mathrm{~m}, 1 \mathrm{H}), 1.92-1.62(\mathrm{~m}, 2 \mathrm{H}), 1.50-1.15$ (m, 2H), 1.08-0.80 (m, 6H); HRMS (ESI) calcd for $\mathrm{C}_{28} \mathrm{H}_{30} \mathrm{~F}_{3} \mathrm{~N}_{3} \mathrm{O}[\mathrm{M}+\mathrm{H}]^{+}: 482.2414$. Found: 482.2416. 
$\left(1 R^{\star}, 2 R^{\star}\right)$-2-(Pyridin-2-yl)cyclopropanecarboxylic Acid [(2S,3S)-2-Amino-3methylpentyl]-(4' -ethylbiphenyl-4-yl)amide (5c)_The procedure for $4 \mathbf{4 a}$ was followed using $20 \mathrm{mg}(0.037 \mathrm{mmol})$ of $\mathbf{1 0 c}$ and $1 \mathrm{~mL}$ of $4 \mathrm{M} \mathrm{HCl}$ in dioxane to give $18 \mathrm{mg}$ (95\%) of $\mathbf{5 c}$ dihydrochloride as a 1:1 diastereomeric mixture: ${ }^{1} \mathrm{H}$ NMR $\left(300 \mathrm{MHz} ; \mathrm{CD}_{3} \mathrm{OD}\right)$ $\delta 8.70-8.58(\mathrm{~m}, 1 \mathrm{H}), 8.45-8.30(\mathrm{~m}, 1 \mathrm{H}), 7.88-7.45(\mathrm{~m}, 8 \mathrm{H}), 7.32-7.20(\mathrm{~m}, 2 \mathrm{H}), 4.52-4.40$ $(\mathrm{m}, 0.5 \mathrm{H}), 4.38-4.28(\mathrm{~m}, 0.5 \mathrm{H}), 3.88-3.56(\mathrm{~m}, 1 \mathrm{H}), 3.48-3.35(\mathrm{~m}, 1 \mathrm{H}), 3.15-3.05(\mathrm{~m}$, $0.5 \mathrm{H}), 3.05-2.95(\mathrm{~m}, 0.5 \mathrm{H}), 2.80-2.60(\mathrm{~m}, 2 \mathrm{H}), 2.25-2.10(\mathrm{~m}, 1 \mathrm{H}), 2.10-1.90(\mathrm{~m}, 1 \mathrm{H})$, $1.87-1.60(\mathrm{~m}, 2 \mathrm{H}), 1.50-1.10(\mathrm{~m}, 5 \mathrm{H}), 1.08-0.80(\mathrm{~m}, 6 \mathrm{H})$; HRMS (ESI) calcd for $\mathrm{C}_{29} \mathrm{H}_{35} \mathrm{~N}_{3} \mathrm{O}[\mathrm{M}+\mathrm{H}]^{+}:$442.2853. Found: 442.2867 .

$\left(1 R^{\star}, 2 R^{\star}\right)$-2-(Pyridin-2-yl)cyclopropanecarboxylic Acid [(2S,3S)-2-Amino-3methylpentyl]-(4'-isopropylbiphenyl-4-yl)amide (5d)_-The procedure for $4 \mathbf{4}$ was followed using $45 \mathrm{mg}(0.08 \mathrm{mmol})$ of $\mathbf{1 0 d}$ and $2 \mathrm{~mL}$ of $4 \mathrm{M} \mathrm{HCl}$ in dioxane to give $40 \mathrm{mg}$ (95\%) of $\mathbf{5 d}$ dihydrochloride as a 1:1 diastereomeric mixture: ${ }^{1} \mathrm{H}$ NMR $\left(300 \mathrm{MHz} ; \mathrm{CD}_{3} \mathrm{OD}\right)$ $\delta 8.60-8.50(\mathrm{~m}, 1 \mathrm{H}), 8.30-8.18(\mathrm{~m}, 1 \mathrm{H}), 7.70-7.48(\mathrm{~m}, 8 \mathrm{H}), 7.36-7.28(\mathrm{~m}, 2 \mathrm{H}), 4.48-4.22$ (m, 1H), 3.83-3.62 (m, 1H), 3.46-3.38 (m, 1H), 3.06-2.88 (m, 2H), 2.20-2.10 (m, 1H), 2.00-1.84 (m, 1H), 1.84-1.72 (m, 1H), 1.72-1.60 (m, 1H), 1.50-1.20 (m, 2H), $1.28(\mathrm{~d}, J=$ $9.0 \mathrm{~Hz}, 6 \mathrm{H}), 1.05-0.80(\mathrm{~m}, 6 \mathrm{H})$; HRMS (ESI) calcd for $\mathrm{C}_{30} \mathrm{H}_{37} \mathrm{~N}_{3} \mathrm{O}[\mathrm{M}+\mathrm{H}]^{+}: 456.3009$. Found: 456.3014.

$\left(1 R^{\star}, 2 R^{\star}\right)$-2-(Pyridin-2-yl)cyclopropanecarboxylic Acid [(2S,3S)-2-Amino-3methylpentyl]-(4' -isobutylbiphenyl-4-yl)amide (5e)—The procedure for $4 \mathbf{a}$ was followed using $20 \mathrm{mg}(0.035 \mathrm{mmol})$ of $10 \mathrm{e}$ and $1 \mathrm{~mL}$ of $4 \mathrm{M} \mathrm{HCl}$ in dioxane to give $17 \mathrm{mg}$ $(90 \%)$ of $5 e$ dihydrochloride as a 1:1 diastereomeric mixture: ${ }^{1} \mathrm{H}$ NMR $\left(300 \mathrm{MHz} ; \mathrm{CD}_{3} \mathrm{OD}\right)$ $\delta 8.68-8.58(\mathrm{~m}, 1 \mathrm{H}), 8.40-8.28(\mathrm{~m}, 1 \mathrm{H}), 7.92-7.43(\mathrm{~m}, 8 \mathrm{H}), 7.30-7.20(\mathrm{~m}, 2 \mathrm{H}), 4.50-4.40$ $(\mathrm{m}, 0.5 \mathrm{H}), 4.38-4.25(\mathrm{~m}, 0.5 \mathrm{H}), 3.88-3.60(\mathrm{~m}, 1 \mathrm{H}), 3.50-3.38(\mathrm{~m}, 1 \mathrm{H}), 3.13-3.05(\mathrm{~m}$, $0.5 \mathrm{H}), 3.05-2.90(\mathrm{~m}, 0.5 \mathrm{H}), 2.51(\mathrm{~d}, J=6.0 \mathrm{~Hz}, 2 \mathrm{H}), 2.25-2.10(\mathrm{~m}, 1 \mathrm{H}), 2.05-1.60(\mathrm{~m}$, $4 \mathrm{H}), 1.50-1.20(\mathrm{~m}, 2 \mathrm{H}), 1.10-0.76(\mathrm{~m}, 12 \mathrm{H})$; HRMS (ESI) calcd for $\mathrm{C}_{31} \mathrm{H}_{39} \mathrm{~N}_{3} \mathrm{O}[\mathrm{M}+\mathrm{H}]^{+}$: 470.3166. Found: 470.3178.

$\left(1 R^{\star}, 2 R^{\star}\right)$-2-(Pyridin-2-yl)cyclopropanecarboxylic Acid [(2S,3S)-2-Amino-3methylpentyl]-(4' -tert-butylbiphenyl-4-yl)amide (5f)—The procedure for $\mathbf{4 a}$ was followed using $20 \mathrm{mg}(0.035 \mathrm{mmol})$ of $\mathbf{1 0 f}$ and $1 \mathrm{~mL}$ of $4 \mathrm{M} \mathrm{HCl}$ in dioxane to give $18 \mathrm{mg}$ $(95 \%)$ of $\mathbf{5 f}$ dihydrochloride as a 1:1 diastereomeric mixture: ${ }^{1} \mathrm{H}$ NMR (300 $\left.\mathrm{MHz} ; \mathrm{CD}_{3} \mathrm{OD}\right)$ $\delta 8.68-8.58(\mathrm{~m}, 1 \mathrm{H}), 8.42-8.30(\mathrm{~m}, 1 \mathrm{H}), 7.88-7.46(\mathrm{~m}, 10 \mathrm{H}), 4.50-4.38(\mathrm{~m}, 0.5 \mathrm{H})$, 4.38-4.22 (m, 0.5H), 3.88-3.60 (m, 1H), 3.46-3.38 (m, 1H), 3.14-3.05 (m, 0.5H), $3.05-2.94(\mathrm{~m}, 0.5 \mathrm{H}), 2.22-2.08(\mathrm{~m}, 1 \mathrm{H}), 2.08-1.90(\mathrm{~m}, 1 \mathrm{H}), 1.88-1.65(\mathrm{~m}, 2 \mathrm{H}), 1.50-1.20$ $(\mathrm{m}, 2 \mathrm{H}), 1.35(\mathrm{~s}, 9 \mathrm{H}), 1.05-0.80(\mathrm{~m}, 6 \mathrm{H})$; HRMS (ESI) calcd for $\mathrm{C}_{31} \mathrm{H}_{39} \mathrm{~N}_{3} \mathrm{O}[\mathrm{M}+\mathrm{H}]^{+}$: 470.3166. Found: 470.3178.

$\left(1 R^{\star}, 2 R^{\star}\right)$-2-(Pyridin-2-yl)cyclopropanecarboxylic Acid [(2S,3S)-2-Amino-3methylpentyl]-(4' -hexylbiphenyl-4-yl)amide (5g)_The procedure for $\mathbf{4 a}$ was followed using $35 \mathrm{mg}(0.058 \mathrm{mmol})$ of $\mathbf{1 0 g}$ and $1 \mathrm{~mL}$ of $4 \mathrm{M} \mathrm{HCl}$ in dioxane to give $30 \mathrm{mg}$ $(90 \%)$ of $\mathbf{5 g}$ dihydrochloride as a 1:1 diastereomeric mixture: ${ }^{1} \mathrm{H}$ NMR $\left(300 \mathrm{MHz} ; \mathrm{CD}_{3} \mathrm{OD}\right)$ S 8.52-8.42 (m, 1H), 8.22-8.10 (m, 1H), 7.65-7.32 (m, 8H), 7.25-7.10 (m, 2H), 4.40-4.28 
(m, 0.5H), 4.28-4.16 (m, 0.5H), 3.75-3.55 (m, 1H), 3.36-3.25 (m, 1H), 2.98-2.86 (m, $0.5 \mathrm{H}), 2.86-2.68(\mathrm{~m}, 0.5 \mathrm{H}), 2.60-2.45(\mathrm{~m}, 2 \mathrm{H}), 2.10-1.95(\mathrm{~m}, 1 \mathrm{H}), 1.90-1.60(\mathrm{~m}, 2 \mathrm{H})$, 1.60-1.45 (m, 3H), 1.40-1.05 (m, 8H), 0.95-0.68 (m, 9H); HRMS (ESI) calcd for $\mathrm{C}_{33} \mathrm{H}_{43} \mathrm{~N}_{3} \mathrm{O}[\mathrm{M}+\mathrm{H}]^{+}:$498.3479. Found: 498.3496 .

$\left(1 R^{\star}, 2 R^{\star}\right)$-2-(Pyridin-2-yl)cyclopropanecarboxylic Acid [(2S,3S)-2-Amino-3methylpentyl]-(4' -cyclohexylbiphenyl-4-yl)amide (5h)-The procedure for $\mathbf{4 a}$ was followed using $20 \mathrm{mg}(0.034 \mathrm{mmol})$ of $\mathbf{1 0 h}$ and $1 \mathrm{~mL}$ of $4 \mathrm{M} \mathrm{HCl}$ in dioxane to give $19 \mathrm{mg}$ (98\%) of $\mathbf{5 h}$ dihydrochloride as a 1:1 diastereomeric mixture: ${ }^{1} \mathrm{H}$ NMR $\left(300 \mathrm{MHz} ; \mathrm{CD}_{3} \mathrm{OD}\right)$ $\delta 8.55-8.40(\mathrm{~m}, 1 \mathrm{H}), 8.15-8.00(\mathrm{~m}, 1 \mathrm{H}), 7.75-7.40(\mathrm{~m}, 8 \mathrm{H}), 7.35-7.20(\mathrm{~m}, 2 \mathrm{H}), 4.48-4.20$ (m, 1H), 3.88-3.68 (m, 1H), 3.42-3.35 (m, 1H), 2.98-2.88 (m, 1H), 2.68-2.48 (m, 1H), 2.20-2.00 (m, 1H), 1.98-1.70 (m, 7H), 1.66-1.10 (m, 8H), 1.05-0.78 (m, 6H); HRMS (ESI) calcd for $\mathrm{C}_{33} \mathrm{H}_{41} \mathrm{~N}_{3} \mathrm{O}[\mathrm{M}+\mathrm{H}]^{+}$: 496.3322. Found: 496.3323 .

$\left(1 R^{\star}, 2 R^{\star}\right)$-2-(Pyridin-2-yl)cyclopropanecarboxylic Acid [(2S,3S)-2-Amino-3methylpentyl]-(4'-phenylbiphenyl-4-yl)amide (5i)_The procedure for $\mathbf{4 a}$ was followed using $22 \mathrm{mg}(0.037 \mathrm{mmol})$ of $\mathbf{1 0 i}$ and $1 \mathrm{~mL}$ of $4 \mathrm{M} \mathrm{HCl}$ in dioxane to give $20 \mathrm{mg}$ (95\%) of $\mathbf{5 i}$ dihydrochloride as a 1:1 diastereomeric mixture: ${ }^{1} \mathrm{H}$ NMR $\left(300 \mathrm{MHz} ; \mathrm{CD}_{3} \mathrm{OD}\right)$ $\delta 8.70-8.58(\mathrm{~m}, 1 \mathrm{H}), 8.42-8.30(\mathrm{~m}, 1 \mathrm{H}), 7.88-7.52(\mathrm{~m}, 12 \mathrm{H}), 7.50-7.30(\mathrm{~m}, 3 \mathrm{H})$, $4.52-4.40(\mathrm{~m}, 0.5 \mathrm{H}), 4.40-4.25(\mathrm{~m}, 0.5 \mathrm{H}), 3.90-3.60(\mathrm{~m}, 1 \mathrm{H}), 3.38-3.48(\mathrm{~m}, 1 \mathrm{H})$, $3.16-3.06(\mathrm{~m}, 0.5 \mathrm{H}), 3.06-2.96(\mathrm{~m}, 0.5 \mathrm{H}), 2.26-2.10(\mathrm{~m}, 1 \mathrm{H}), 2.10-1.90(\mathrm{~m}, 1 \mathrm{H})$, 1.90-1.68 (m, 2H), 1.52-1.20 (m, 2H), 1.00-0.80 (m, 6H); HRMS (ESI) calcd for $\mathrm{C}_{33} \mathrm{H}_{35} \mathrm{~N}_{3} \mathrm{O}[\mathrm{M}+\mathrm{H}]^{+}:$490.2853. Found: 490.2870 .

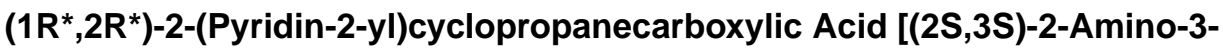
methylpentyl]-(4' -methoxybiphenyl-4-yl)amide (5j)—The procedure for $\mathbf{4 a}$ was followed using $36 \mathrm{mg}(0.066 \mathrm{mmol})$ of $\mathbf{1 0 j}$ and $2 \mathrm{~mL}$ of $4 \mathrm{M} \mathrm{HCl}$ in dioxane to give $32 \mathrm{mg}$ (94\%) of $\mathbf{5 j}$ dihydrochloride as a 1:1 diastereomeric mixture: ${ }^{1} \mathrm{H}$ NMR $\left(300 \mathrm{MHz} ; \mathrm{CD}_{3} \mathrm{OD}\right)$ $\delta 8.60-8.52(\mathrm{~m}, 1 \mathrm{H}), 8.32-8.20(\mathrm{~m}, 1 \mathrm{H}), 7.76-7.48(\mathrm{~m}, 8 \mathrm{H}), 7.05-6.96(\mathrm{~m}, 2 \mathrm{H}), 4.46-4.26$ $(\mathrm{m}, 1 \mathrm{H}), 3.83(\mathrm{~s}, 3 \mathrm{H}), 3.80-3.62(\mathrm{~m}, 1 \mathrm{H}), 3.45-3.32(\mathrm{~m}, 1 \mathrm{H}), 3.10-2.98(\mathrm{~m}, 0.5 \mathrm{H})$, $2.98-2.88(\mathrm{~m}, 0.5 \mathrm{H}), 2.22-2.06(\mathrm{~m}, 1 \mathrm{H}), 2.00-1.82(\mathrm{~m}, 1 \mathrm{H}), 1.82-1.58(\mathrm{~m}, 2 \mathrm{H}), 1.46-1.15$ (m, 2H), 1.05-0.75 (m, 6H); HRMS (ESI) calcd for $\mathrm{C}_{28} \mathrm{H}_{33} \mathrm{~N}_{3} \mathrm{O}_{2}[\mathrm{M}+\mathrm{H}]^{+}: 444.2646$. Found: 444.2655 .

$\left(1 R^{\star}, 2 R^{\star}\right)$-2-(Pyridin-2-yl)cyclopropanecarboxylic Acid [(2S,3S)-2-Amino-3methylpentyl]-(4' -trifluoromethoxybiphenyl-4-yl)amide (5k)—The procedure for 4a was followed using $18 \mathrm{mg}(0.03 \mathrm{mmol})$ of $10 \mathrm{k}$ and $1 \mathrm{~mL}$ of $4 \mathrm{M} \mathrm{HCl}$ in dioxane to give $16 \mathrm{mg}(94 \%)$ of $\mathbf{5 k}$ dihydrochloride as a 1:1 diastereomeric mixture: ${ }^{1} \mathrm{H}$ NMR (300 $\mathrm{MHz}$; $\left.\mathrm{CD}_{3} \mathrm{OD}\right) \delta 8.68-8.56(\mathrm{~m}, 1 \mathrm{H}), 8.45-8.30(\mathrm{~m}, 1 \mathrm{H}), 7.90-7.56(\mathrm{~m}, 8 \mathrm{H}), 7.42-7.30(\mathrm{~m}, 2 \mathrm{H})$, $4.52-4.40(\mathrm{~m}, 0.5 \mathrm{H}), 4.38-4.22(\mathrm{~m}, 0.5 \mathrm{H}), 3.90-3.60(\mathrm{~m}, 1 \mathrm{H}), 3.48-3.38(\mathrm{~m}, 1 \mathrm{H})$, 3.15-3.06 (m, 0.5H), 3.06-2.95 (m, 0.5H), 2.22-2.08 (m, 1H), 2.08-1.90 (m, 1H), 1.90-1.64 (m, 2H), 1.50-1.16 (m, 2H), 1.06-0.78 (m, 6H); HRMS (ESI) calcd for $\mathrm{C}_{28} \mathrm{H}_{30} \mathrm{~F}_{3} \mathrm{~N}_{3} \mathrm{O}_{2}[\mathrm{M}+\mathrm{H}]^{+}:$498.2363. Found: 498.2385 . 
$\left(1 R^{\star}, 2 R^{\star}\right)$-2-(Pyridin-2-yl)cyclopropanecarboxylic Acid [(2S,3S)-2-Amino-3methylpentyl]-(4' -ethoxybiphenyl-4-yl)amide (5I)—The procedure for $4 a$ was followed using $22 \mathrm{mg}(0.039 \mathrm{mmol})$ of $\mathbf{1 0 l}$ and $1 \mathrm{~mL}$ of $4 \mathrm{M} \mathrm{HCl}$ in dioxane to give $21 \mathrm{mg}$ $(100 \%)$ of $\mathbf{5 l}$ dihydrochloride as a 1:1 diastereomeric mixture: ${ }^{1} \mathrm{H}$ NMR $(300 \mathrm{MHz}$; $\left.\mathrm{CD}_{3} \mathrm{OD}\right) \delta 8.70-8.58(\mathrm{~m}, 1 \mathrm{H}), 8.42-8.30(\mathrm{~m}, 1 \mathrm{H}), 7.88-7.76(\mathrm{~m}, 1 \mathrm{H}), 7.76-7.38(\mathrm{~m}, 7 \mathrm{H})$, $7.02-6.90(\mathrm{~m}, 2 \mathrm{H}), 4.52-4.40(\mathrm{~m}, 0.5 \mathrm{H}), 4.38-4.24(\mathrm{~m}, 0.5 \mathrm{H}), 4.07(\mathrm{q}, J=6.0 \mathrm{~Hz}, 2 \mathrm{H})$, $3.86-3.55(\mathrm{~m}, 1 \mathrm{H}), 3.46-3.33(\mathrm{~m}, 1 \mathrm{H}), 3.15-3.05(\mathrm{~m}, 0.5 \mathrm{H}), 3.05-2.92(\mathrm{~m}, 0.5 \mathrm{H})$, 2.25-2.08 (m, 1H), 2.08-1.90 (m, 1H), 1.90-1.60 (m, 2H), 1.50-1.16 (m, 5H), 1.08-0.78 (m, 6H); HRMS (ESI) calcd for $\mathrm{C}_{29} \mathrm{H}_{35} \mathrm{~N}_{3} \mathrm{O}_{2}[\mathrm{M}+\mathrm{H}]^{+}$: 458.2802. Found: 458.2812.

$\left(1 R^{\star}, 2 R^{\star}\right)$-2-(Pyridin-2-yl)cyclopropanecarboxylic Acid [(2S,3S)-2-Amino-3methylpentyl]-(4'-isopropoxybiphenyl-4-yl)amide $(5 \mathrm{~m})$-The procedure for $4 \mathrm{a}$ was followed using $20 \mathrm{mg}(0.035 \mathrm{mmol})$ of $\mathbf{1 0 m}$ and $1 \mathrm{~mL}$ of $4 \mathrm{M} \mathrm{HCl}$ in dioxane to give $19 \mathrm{mg}$ $(100 \%)$ of $\mathbf{5 m}$ dihydrochloride as a 1:1 diastereomeric mixture: ${ }^{1} \mathrm{H}$ NMR $(300 \mathrm{MHz}$; $\left.\mathrm{CD}_{3} \mathrm{OD}\right) \delta 8.70-8.55(\mathrm{~m}, 1 \mathrm{H}), 8.40-8.30(\mathrm{~m}, 1 \mathrm{H}), 7.90-7.40(\mathrm{~m}, 8 \mathrm{H}), 7.05-6.90(\mathrm{~m}, 2 \mathrm{H})$, $4.70-4.58(\mathrm{~m}, 1 \mathrm{H}), 4.55-4.38(\mathrm{~m}, 0.5 \mathrm{H}), 4.38-4.22(\mathrm{~m}, 0.5 \mathrm{H}), 3.85-3.60(\mathrm{~m}, 1 \mathrm{H})$, $3.46-3.38(\mathrm{~m}, 1 \mathrm{H}), 3.16-3.05(\mathrm{~m}, 0.5 \mathrm{H}), 3.05-2.94(\mathrm{~m}, 0.5 \mathrm{H}), 2.25-2.08(\mathrm{~m}, 1 \mathrm{H})$, 2.08-1.90 (m, 1H), 1.90-1.64 (m, 2H), 1.50-1.20 (m, 2H), $1.33(\mathrm{~d}, J=6.0 \mathrm{~Hz}, 6 \mathrm{H})$, 1.06-0.80 (m, 6H); HRMS (ESI) calcd for $\mathrm{C}_{30} \mathrm{H}_{37} \mathrm{~N}_{3} \mathrm{O}_{2}[\mathrm{M}+\mathrm{H}]^{+}: 472.2959$. Found: 472.2957.

$\left(1 R^{\star}, 2 R^{\star}\right)$-2-(Pyridin-2-yl)cyclopropanecarboxylic Acid [(2S,3S)-2-Amino-3methylpentyl]-(3', $\mathbf{4}^{\prime}$-dimethoxybiphenyl-4-yl)amide (5n)—The procedure for $\mathbf{4 a}$ was followed using $19 \mathrm{mg}(0.033 \mathrm{mmol})$ of $\mathbf{1 0 n}$ and $1 \mathrm{~mL}$ of $4 \mathrm{M} \mathrm{HCl}$ in dioxane to give 18 $\mathrm{mg}(100 \%)$ of $\mathbf{5 n}$ dihydrochloride as a 1:1 diastereomeric mixture: ${ }^{1} \mathrm{H}$ NMR (300 MHz; $\left.\mathrm{CD}_{3} \mathrm{OD}\right) \delta 8.68-8.58(\mathrm{~m}, 1 \mathrm{H}), 8.46-8.32(\mathrm{~m}, 1 \mathrm{H}), 7.90-7.78(\mathrm{~m}, 1 \mathrm{H}), 7.78-7.53(\mathrm{~m}, 5 \mathrm{H})$, 7.20-7.10 (m, 2H), 7.08-6.98 (m, 1H), 4.52-4.40 (m, 0.5H), 4.40-4.25 (m, 0.5H), 3.89 (s, $3 \mathrm{H}), 3.86(\mathrm{~s}, 3 \mathrm{H}), 3.80-3.62(\mathrm{~m}, 1 \mathrm{H}), 3.46-3.35(\mathrm{~m}, 1 \mathrm{H}), 3.15-3.06(\mathrm{~m}, 0.5 \mathrm{H}), 3.06-2.96$ (m, 0.5H), 2.25-2.10 (m, 1H), 2.10-1.90 (m, 1H), 1.90-1.65 (m, 2H), 1.50-1.18 (m, 2H), 1.05-0.80 (m, 6H); HRMS (ESI) calcd for $\mathrm{C}_{29} \mathrm{H}_{35} \mathrm{~N}_{3} \mathrm{O}_{3}[\mathrm{M}+\mathrm{H}]^{+}: 474.2751$. Found: 474.2763.

$\left(1 R^{\star}, 2 R^{\star}\right)$-2-(Pyridin-2-yl)cyclopropanecarboxylic Acid [(2S,3S)-2-Amino-3methylpentyl]-( $3^{\prime}, 4^{\prime}$-methylenedioxybiphenyl-4-yl)amide (50)-The procedure for 4a was followed using $20 \mathrm{mg}(0.036 \mathrm{mmol})$ of $\mathbf{1 0 o}$ and $1 \mathrm{~mL}$ of $4 \mathrm{M} \mathrm{HCl}$ in dioxane to give $19 \mathrm{mg}(100 \%)$ of $\mathbf{5 o}$ dihydrochloride as a 1:1 diastereomeric mixture: ${ }^{1} \mathrm{H}$ NMR (300 MHz; $\left.\mathrm{CD}_{3} \mathrm{OD}\right) \delta 8.68-8.56(\mathrm{~m}, 1 \mathrm{H}), 8.42-8.28(\mathrm{~m}, 1 \mathrm{H}), 7.85-7.73(\mathrm{~m}, 1 \mathrm{H}), 7.73-7.50(\mathrm{~m}, 5 \mathrm{H})$, 7.12-7.00 (m, 2H), 6.92-6.82 (m, 1H), $5.99(\mathrm{~s}, 2 \mathrm{H}), 4.50-4.35(\mathrm{~m}, 0.5 \mathrm{H}), 4.34-4.20(\mathrm{~m}$, $0.5 \mathrm{H}), 3.85-3.60(\mathrm{~m}, 1 \mathrm{H}), 3.45-3.32(\mathrm{~m}, 1 \mathrm{H}), 3.12-3.02(\mathrm{~m}, 0.5 \mathrm{H}), 3.02-2.90(\mathrm{~m}, 0.5 \mathrm{H})$, $2.22-2.08(\mathrm{~m}, 1 \mathrm{H}), 2.08-1.88(\mathrm{~m}, 1 \mathrm{H}), 1.88-1.60(\mathrm{~m}, 2 \mathrm{H}), 1.50-1.15(\mathrm{~m}, 2 \mathrm{H}), 1.06-0.78$ $\left(\mathrm{m}, 6 \mathrm{H}\right.$ ); HRMS (ESI) calcd for $\mathrm{C}_{28} \mathrm{H}_{31} \mathrm{~N}_{3} \mathrm{O}_{3}[\mathrm{M}+\mathrm{H}]^{+}$: 458.2438. Found: 458.2458.

$\left(1 R^{\star}, 2 R^{\star}\right)$-2-(Pyridin-2-yl)cyclopropanecarboxylic Acid [(2S,3S)-2-Amino-3methylpentyl]-(4'-fluorobiphenyl-4-yl)amide (5p)-The procedure for $4 \mathbf{a}$ was followed using $20 \mathrm{mg}(0.038 \mathrm{mmol})$ of $\mathbf{1 0 p}$ and $1 \mathrm{~mL}$ of $4 \mathrm{M} \mathrm{HCl}$ in dioxane to give $18 \mathrm{mg}$ 
(94\%) of $\mathbf{5 p}$ dihydrochloride as a 1:1 diastereomeric mixture: ${ }^{1} \mathrm{H}$ NMR (300 MHz; $\left.\mathrm{CD}_{3} \mathrm{OD}\right)$ $\delta 8.70-8.58(\mathrm{~m}, 1 \mathrm{H}), 8.45-8.35(\mathrm{~m}, 1 \mathrm{H}), 7.90-7.50(\mathrm{~m}, 8 \mathrm{H}), 7.25-7.10(\mathrm{~m}, 2 \mathrm{H}), 4.52-4.40$ $(\mathrm{m}, 0.5 \mathrm{H}), 4.40-4.25(\mathrm{~m}, 0.5 \mathrm{H}), 3.90-3.60(\mathrm{~m}, 1 \mathrm{H}), 3.52-3.40(\mathrm{~m}, 1 \mathrm{H}), 3.16-3.06(\mathrm{~m}$, $0.5 \mathrm{H}), 3.05-2.92(\mathrm{~m}, 0.5 \mathrm{H}), 2.25-2.10(\mathrm{~m}, 1 \mathrm{H}), 2.10-1.92(\mathrm{~m}, 1 \mathrm{H}), 1.92-1.60(\mathrm{~m}, 2 \mathrm{H})$, $1.50-1.18(\mathrm{~m}, 2 \mathrm{H}), 1.10-0.80(\mathrm{~m}, 6 \mathrm{H})$; HRMS (ESI) calcd for $\mathrm{C}_{27} \mathrm{H}_{30} \mathrm{FN}_{3} \mathrm{O}[\mathrm{M}+\mathrm{H}]^{+}$: 432.2446. Found: 432.2453.

$\left(1 R^{\star}, 2 R^{\star}\right)$-2-(Pyridin-2-yl)cyclopropanecarboxylic Acid [(2S,3S)-2-Amino-3methylpentyl]-(4' -chlorobiphenyl-4-yl)amide (5q)-The procedure for $\mathbf{4 a}$ was followed using $20 \mathrm{mg}(0.036 \mathrm{mmol})$ of $\mathbf{1 0 q}$ and $1 \mathrm{~mL}$ of $4 \mathrm{M} \mathrm{HCl}$ in dioxane to give $18 \mathrm{mg}$ (96\%) of $\mathbf{5 q}$ dihydrochloride as a 1:1 diastereomeric mixture: ${ }^{1} \mathrm{H}$ NMR $\left(300 \mathrm{MHz} ; \mathrm{CD}_{3} \mathrm{OD}\right)$ $\delta 8.72-8.58(\mathrm{~m}, 1 \mathrm{H}), 8.45-8.35(\mathrm{~m}, 1 \mathrm{H}), 7.90-7.55(\mathrm{~m}, 8 \mathrm{H}), 7.55-7.40(\mathrm{~m}, 2 \mathrm{H}), 4.55-4.42$ $(\mathrm{m}, 0.5 \mathrm{H}), 4.40-4.25(\mathrm{~m}, 0.5 \mathrm{H}), 3.90-3.60(\mathrm{~m}, 1 \mathrm{H}), 3.50-3.40(\mathrm{~m}, 1 \mathrm{H}), 3.18-3.07(\mathrm{~m}$, $0.5 \mathrm{H}), 3.06-2.96(\mathrm{~m}, 0.5 \mathrm{H}), 2.25-2.10(\mathrm{~m}, 1 \mathrm{H}), 2.08-1.90(\mathrm{~m}, 1 \mathrm{H}), 1.90-1.68(\mathrm{~m}, 2 \mathrm{H})$, 1.58-1.12 (m, 2H), 1.10-0.80 (m, 6H); HRMS (ESI) calcd for $\mathrm{C}_{27} \mathrm{H}_{30} \mathrm{ClN}_{3} \mathrm{O}[\mathrm{M}+\mathrm{H}]^{+}$: 448.2150. Found: 448.2162.

$\left(1 R^{\star}, 2 R^{\star}\right)$-2-(Pyridin-2-yl)cyclopropanecarboxylic Acid [(2S,3S)-2-Amino-3methylpentyl]-(4' -cyanobiphenyl-4-yl)amide (5r)—The procedure for $\mathbf{4 a}$ was followed using $20 \mathrm{mg}(0.037 \mathrm{mmol})$ of 10r and $1 \mathrm{~mL}$ of $4 \mathrm{M} \mathrm{HCl}$ in dioxane to give $18 \mathrm{mg}$ $(95 \%)$ of $\mathbf{5 r}$ dihydrochloride as a 1:1 diastereomeric mixture: ${ }^{1} \mathrm{H}$ NMR $\left(300 \mathrm{MHz} ; \mathrm{CD}_{3} \mathrm{OD}\right)$ $\delta 8.65-8.55(\mathrm{~m}, 1 \mathrm{H}), 8.36-8.25(\mathrm{~m}, 1 \mathrm{H}), 7.90-7.50(\mathrm{~m}, 10 \mathrm{H}), 4.50-4.38(\mathrm{~m}, 0.5 \mathrm{H})$, $4.38-4.20(\mathrm{~m}, 0.5 \mathrm{H}), 3.85-3.60(\mathrm{~m}, 1 \mathrm{H}), 3.45-3.28(\mathrm{~m}, 1 \mathrm{H}), 3.12-3.02(\mathrm{~m}, 0.5 \mathrm{H})$, $3.02-2.92(\mathrm{~m}, 0.5 \mathrm{H}), 2.22-2.06(\mathrm{~m}, 1 \mathrm{H}), 2.06-1.90(\mathrm{~m}, 1 \mathrm{H}), 1.90-1.60(\mathrm{~m}, 2 \mathrm{H}), 1.58-1.15$ (m, 2H), 1.05-0.80 (m, 6H); HRMS (ESI) calcd for $\mathrm{C}_{28} \mathrm{H}_{30} \mathrm{~N}_{4} \mathrm{O}[\mathrm{M}+\mathrm{H}]^{+}: 439.2492$. Found: 439.2506.

$\left(1 R^{\star}, 2 R^{\star}\right)$-2-(Pyridin-2-yl)cyclopropanecarboxylic Acid [(2S,3S)-2-Amino-3methylpentyl]-(4' -nitrobiphenyl-4-yl)amide (5s) - The procedure for $4 \mathbf{a}$ was followed using $21 \mathrm{mg}(0.037 \mathrm{mmol})$ of $\mathbf{1 0 s}$ and $1 \mathrm{~mL}$ of $4 \mathrm{M} \mathrm{HCl}$ in dioxane to give $19 \mathrm{mg}(95 \%)$ of 5s dihydrochloride as a $1: 1$ diastereomeric mixture: ${ }^{1} \mathrm{H}$ NMR $\left(300 \mathrm{MHz} ; \mathrm{CD}_{3} \mathrm{OD}\right) \delta$ 8.65-8.55 (m, 1H), 8.45-8.20 (m, 2H), 7.95-7.55 (m, 9H), 4.50-4.30 (m, 1H), 3.85-3.60 (m, $1 \mathrm{H}), 3.45-3.35(\mathrm{~m}, 1 \mathrm{H}), 3.12-3.02(\mathrm{~m}, 0.5 \mathrm{H}), 3.02-2.95(\mathrm{~m}, 0.5 \mathrm{H}), 2.25-2.10(\mathrm{~m}, 1 \mathrm{H})$, 2.10-1.90 (m, 1H), 1.89-1.60 (m, 2H), 1.50-1.15 (m, 2H), 1.10-0.82 (m, 6H); HRMS (ESI) calcd for $\mathrm{C}_{27} \mathrm{H}_{30} \mathrm{~N}_{4} \mathrm{O}_{3}[\mathrm{M}+\mathrm{H}]^{+}$: 459.2391. Found: 459.2404 .

$\left(1 R^{\star}, 2 R^{\star}\right)$-2-(Pyridin-2-yl)cyclopropanecarboxylic Acid [(2S,3S)-2-Amino-3methylpentyl]-(4' -acetylbiphenyl-4-yl)amide (5t)-The procedure for $\mathbf{4 a}$ was followed using $20 \mathrm{mg}(0.036 \mathrm{mmol})$ of $\mathbf{1 0 t}$ and $1 \mathrm{~mL}$ of $4 \mathrm{M} \mathrm{HCl}$ in dioxane to give $17 \mathrm{mg}$ (90\%) of $\mathbf{5 t}$ dihydrochloride as a 1:1 diastereomeric mixture: ${ }^{1} \mathrm{H} \mathrm{NMR}\left(300 \mathrm{MHz} ; \mathrm{CD}_{3} \mathrm{OD}\right)$ 8 8.35-8.22 (m, 1H), 8.05-7.92 (m, 2H), 7.90-7.75 (m, 1H), 7.72-7.60 (m, 4H), 7.52-7.40 (m, 2H), 7.35-7.22 (m, 2H), 4.38-4.16 (m, 1H), 3.80-3.55 (m, 1H), 3.38-3.20 (m, 1H), 2.80-2.60 (m, 1H), 2.54 (s, 3H), 2.00-1.88 (m, 1H), 1.78-1.60 (m, 2H), 1.50-1.10 (m, 3H), 0.95-0.70 (m, 6H); HRMS (ESI) calcd for $\mathrm{C}_{29} \mathrm{H}_{33} \mathrm{~N}_{3} \mathrm{O}_{2}[\mathrm{M}+\mathrm{H}]^{+}: 456.2646$. Found: 456.2658. 
$\left(1 R^{\star}, 2 R^{\star}\right)$-2-(Pyridin-2-yl)cyclopropanecarboxylic Acid [(2S,3S)-2-Amino-3methylpentyl]-( $\mathbf{4}^{\prime}$-ethoxycarbonylbiphenyl-4-yl)amide (5u)-The procedure for 4 a was followed using $22 \mathrm{mg}(0.038 \mathrm{mmol})$ of $10 \mathrm{u}$ and $1 \mathrm{~mL}$ of $4 \mathrm{M} \mathrm{HCl}$ in dioxane to give 21 $\mathrm{mg}(100 \%)$ of $\mathbf{5 u}$ dihydrochloride as a 1:1 diastereomeric mixture: ${ }^{1} \mathrm{H}$ NMR $(300 \mathrm{MHz}$; $\left.\mathrm{CD}_{3} \mathrm{OD}\right) \delta 8.65-8.48(\mathrm{~m}, 1 \mathrm{H}), 8.35-8.18(\mathrm{~m}, 1 \mathrm{H}), 8.15-8.10(\mathrm{~m}, 2 \mathrm{H}), 7.85-7.50(\mathrm{~m}, 8 \mathrm{H})$, 4.50-4.20 (m, 3H), 3.85-3.62 (m, 1H), 3.45-3.35 (m, 1H), 3.12-3.00 (m, 0.5H), 3.00-2.90 (m, 0.5H), 2.20-2.03 (m, 1H), 2.03-1.55 (m, 3H), 1.45-1.12 (m, 5H), 1.08-0.78 (m, 6H); HRMS (ESI) calcd for $\mathrm{C}_{30} \mathrm{H}_{35} \mathrm{~N}_{3} \mathrm{O}_{3}[\mathrm{M}+\mathrm{H}]^{+}:$486.2751. Found: 486.2774 .

$\left(1 R^{\star}, 2 R^{\star}\right)$-2-(Pyridin-2-yl)cyclopropanecarboxylic Acid [(2S,3S)-2-Amino-3methylpentyl]-(4' -hydroxybiphenyl-4-yl)amide (5v)-The procedure for $4 \mathbf{a}$ was followed using $22 \mathrm{mg}(0.042 \mathrm{mmol})$ of $10 \mathrm{v}$ and $1 \mathrm{~mL}$ of $4 \mathrm{M} \mathrm{HCl}$ in dioxane to give $21 \mathrm{mg}$ $(100 \%)$ of $\mathbf{5 v}$ dihydrochloride as a 1:1 diastereomeric mixture: ${ }^{1} \mathrm{H}$ NMR $(300 \mathrm{MHz}$; $\left.\mathrm{CD}_{3} \mathrm{OD}\right) \delta 8.68-8.55(\mathrm{~m}, 1 \mathrm{H}), 8.40-8.28(\mathrm{~m}, 1 \mathrm{H}), 7.95-7.82(\mathrm{~m}, 1 \mathrm{H}), 7.82-7.38(\mathrm{~m}, 7 \mathrm{H})$, 6.88-6.68 (m, 2H), 4.48-4.36 (m, 0.5H), 4.36-4.22 (m, 0.5H), 3.82-3.65 (m, 1H), $3.45-3.32(\mathrm{~m}, 1 \mathrm{H}), 3.12-3.02(\mathrm{~m}, 0.5 \mathrm{H}), 3.02-2.92(\mathrm{~m}, 0.5 \mathrm{H}), 2.20-2.06(\mathrm{~m}, 1 \mathrm{H})$, 2.06-1.88 (m, 1H), 1.88-1.60 (m, 2H), 1.50-1.15 (m, 2H), 1.05-0.80 (m, 6H); HRMS (ESI) calcd for $\mathrm{C}_{27} \mathrm{H}_{31} \mathrm{~N}_{3} \mathrm{O}_{2}[\mathrm{M}+\mathrm{H}]^{+}$: 430.2489. Found: 430.2508 .

$\left(1 R^{\star}, 2 R^{\star}\right)$-2-(Pyridin-2-yl)cyclopropanecarboxylic Acid [(2S,3S)-2-Amino-3methylpentyl]-(4' -aminobiphenyl-4-yl)amide (5w)-The procedure for $4 \mathbf{4}$ was followed using $22 \mathrm{mg}(0.035 \mathrm{mmol})$ of $\mathbf{1 0 w}$ and $1 \mathrm{~mL}$ of $4 \mathrm{M} \mathrm{HCl}$ in dioxane to give $20 \mathrm{mg}$ $(95 \%)$ of $\mathbf{5 w}$ trihydrochloride as a 1:1 diastereomeric mixture: ${ }^{1} \mathrm{H}$ NMR $(300 \mathrm{MHz}$; $\left.\mathrm{CD}_{3} \mathrm{OD}\right) \delta 8.61-8.55(\mathrm{~m}, 1 \mathrm{H}), 8.40-8.30(\mathrm{~m}, 1 \mathrm{H}), 7.84-7.47(\mathrm{~m}, 10 \mathrm{H}), 4.52-4.42(\mathrm{~m}$, $0.5 \mathrm{H}), 4.40-4.28(\mathrm{~m}, 0.5 \mathrm{H}), 3.80-3.65(\mathrm{~m}, 1 \mathrm{H}), 3.42-3.32(\mathrm{~m}, 1 \mathrm{H}), 3.12-3.00(\mathrm{~m}, 0.5 \mathrm{H})$, $3.00-2.93(\mathrm{~m}, 0.5 \mathrm{H}), 2.22-2.08(\mathrm{~m}, 1 \mathrm{H}), 2.08-1.88(\mathrm{~m}, 1 \mathrm{H}), 1.88-1.60(\mathrm{~m}, 2 \mathrm{H}), 1.50-1.16$ (m, 2H), 1.06-0.80 (m, 6H); HRMS (ESI) calcd for $\mathrm{C}_{27} \mathrm{H}_{32} \mathrm{~N}_{4} \mathrm{O}[\mathrm{M}+\mathrm{H}]^{+}: 429.2649$. Found: 429.2661.

$\left(1 R^{\star}, 2 R^{\star}\right)$-2-(Pyridin-2-yl)cyclopropanecarboxylic Acid [(2S,3S)-2-Amino-3methylpentyl]-[4' -(dimethylamino)biphenyl-4-yl]amide (5x)-The procedure for $\mathbf{4 a}$ was followed using $46 \mathrm{mg}(0.083 \mathrm{mmol})$ of $\mathbf{1 0 x}$ and $2 \mathrm{~mL}$ of $4 \mathrm{M} \mathrm{HCl}$ in dioxane to give 40 $\mathrm{mg}(86 \%)$ of $\mathbf{5 x}$ trihydrochloride as a 1:1 diastereomeric mixture: ${ }^{1} \mathrm{H}$ NMR $(300 \mathrm{MHz}$; $\left.\mathrm{CD}_{3} \mathrm{OD}\right) \delta 8.55-8.45(\mathrm{~m}, 1 \mathrm{H}), 8.25-8.12(\mathrm{~m}, 1 \mathrm{H}), 7.70-7.40(\mathrm{~m}, 10 \mathrm{H}), 4.42-4.28(\mathrm{~m}$, $0.5 \mathrm{H}), 4.28-4.15(\mathrm{~m}, 0.5 \mathrm{H}), 3.82-3.65(\mathrm{~m}, 1 \mathrm{H}), 3.55(\mathrm{~s}, 6 \mathrm{H}), 3.32-3.22(\mathrm{~m}, 1 \mathrm{H}), 3.02-2.90$ (m, 0.5H), 2.90-2.82 (m, 0.5H), 2.10-1.95 (m, 1H), 1.95-1.50 (m, 3H), 1.45-1.10 (m, 2H), 0.95-0.70 (m, 6H); HRMS (ESI) calcd for $\mathrm{C}_{29} \mathrm{H}_{36} \mathrm{~N}_{4} \mathrm{O}[\mathrm{M}+\mathrm{H}]^{+}:$457.2962. Found: 457.2973.

$\left(1 R^{\star}, 2 R^{\star}\right)$-2-(Pyridin-2-yl)cyclopropanecarboxylic Acid [(2S,3S)-2-Amino-3methylpentyl]-(4' -acetamidobiphenyl-4-yl)amide (5y)-The procedure for $4 \mathbf{4 a}$ was followed using $18 \mathrm{mg}(0.032 \mathrm{mmol})$ of $\mathbf{1 0 y}$ and $1 \mathrm{~mL}$ of $4 \mathrm{M} \mathrm{HCl}$ in dioxane to give $17 \mathrm{mg}$ (99\%) of $\mathbf{5 y}$ dihydrochloride as a 1:1 diastereomeric mixture: ${ }^{1} \mathrm{H}$ NMR $\left(300 \mathrm{MHz} ; \mathrm{CD}_{3} \mathrm{OD}\right)$ $\delta 8.68-8.58(\mathrm{~m}, 1 \mathrm{H}), 8.45-8.30(\mathrm{~m}, 1 \mathrm{H}), 7.85-7.50(\mathrm{~m}, 10 \mathrm{H}), 4.50-4.38(\mathrm{~m}, 0.5 \mathrm{H})$, $4.38-4.20(\mathrm{~m}, 0.5 \mathrm{H}), 3.82-3.65(\mathrm{~m}, 1 \mathrm{H}), 3.55-3.35(\mathrm{~m}, 1 \mathrm{H}), 3.12-3.02(\mathrm{~m}, 0.5 \mathrm{H})$, 
3.02-2.90 (m, 0.5H), $2.15(\mathrm{~s}, 3 \mathrm{H}), 2.15-1.96(\mathrm{~m}, 4 \mathrm{H}), 1.50-1.16(\mathrm{~m}, 2 \mathrm{H}), 1.05-0.80(\mathrm{~m}$, $6 \mathrm{H}$ ); HRMS (ESI) calcd for $\mathrm{C}_{29} \mathrm{H}_{34} \mathrm{~N}_{4} \mathrm{O}_{2}[\mathrm{M}+\mathrm{H}]^{+}: 471.2755$. Found: 471.2768 .

$\left(1 R^{\star}, 2 R^{\star}\right)$-2-(Pyridin-2-yl)cyclopropanecarboxylic Acid [(2S,3S)-2-Amino-3methylpentyl]-(4' -carbamoylbiphenyl-4-yl)amide (5z)-The procedure for $\mathbf{4 a}$ was followed using $18 \mathrm{mg}(0.032 \mathrm{mmol})$ of $\mathbf{1 0 z}$ and $1 \mathrm{~mL}$ of $4 \mathrm{M} \mathrm{HCl}$ in dioxane to give $17 \mathrm{mg}$ (99\%) of $\mathbf{5 z}$ dihydrochloride as a 1:1 diastereomeric mixture: ${ }^{1} \mathrm{H}$ NMR $\left(300 \mathrm{MHz} ; \mathrm{CD}_{3} \mathrm{OD}\right)$ $\delta 8.68-8.55(\mathrm{~m}, 1 \mathrm{H}), 8.45-8.30(\mathrm{~m}, 1 \mathrm{H}), 8.00-7.50(\mathrm{~m}, 10 \mathrm{H}), 4.50-4.38(\mathrm{~m}, 0.5 \mathrm{H})$, $4.38-4.22(\mathrm{~m}, 0.5 \mathrm{H}), 3.85-3.65(\mathrm{~m}, 1 \mathrm{H}), 3.46-3.35(\mathrm{~m}, 1 \mathrm{H}), 3.12-3.02(\mathrm{~m}, 0.5 \mathrm{H})$, $3.02-2.92(\mathrm{~m}, 0.5 \mathrm{H}), 2.25-2.10(\mathrm{~m}, 1 \mathrm{H}), 2.10-1.90(\mathrm{~m}, 1 \mathrm{H}), 1.90-1.55(\mathrm{~m}, 2 \mathrm{H}), 1.50-1.16$ (m, 2H), 1.08-0.80 (m, 6H); HRMS (ESI) calcd for $\mathrm{C}_{28} \mathrm{H}_{32} \mathrm{~N}_{4} \mathrm{O}_{2}[\mathrm{M}+\mathrm{H}]^{+}: 457.2598$. Found: 457.2616.

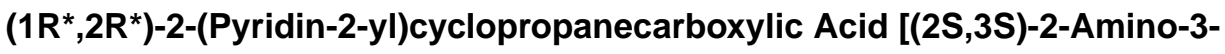
methylpentyl]-[4' -(dimethylcarbamoyl)biphenyl-4-yl]-amide (5aa)-The procedure for $4 \mathbf{a}$ was followed using $20 \mathrm{mg}(0.034 \mathrm{mmol})$ of $\mathbf{1 0 a a}$ and $1 \mathrm{~mL}$ of $4 \mathrm{M} \mathrm{HCl}$ in dioxane to give $19 \mathrm{mg}(100 \%)$ of $\mathbf{5 a a}$ dihydrochloride as a 1:1 diastereomeric mixture: ${ }^{1} \mathrm{H}$ NMR (300 MHz; CD 3 OD) $\delta 8.86-8.55(\mathrm{~m}, 1 \mathrm{H}), 8.50-8.25(\mathrm{~m}, 1 \mathrm{H}), 8.00-7.40(\mathrm{~m}, 10 \mathrm{H})$, $4.50-4.38(\mathrm{~m}, 0.5 \mathrm{H}), 4.38-4.20(\mathrm{~m}, 0.5 \mathrm{H}), 3.85-3.65(\mathrm{~m}, 1 \mathrm{H}), 3.40-3.25(\mathrm{~m}, 1 \mathrm{H})$, $3.20-2.95(\mathrm{~m}, 7 \mathrm{H}), 2.25-2.10(\mathrm{~m}, 1 \mathrm{H}), 2.10-1.60(\mathrm{~m}, 3 \mathrm{H}), 1.50-1.16(\mathrm{~m}, 2 \mathrm{H}), 1.08-0.80$ (m, 6H); HRMS (ESI) calcd for $\mathrm{C}_{30} \mathrm{H}_{36} \mathrm{~N}_{4} \mathrm{O}_{2}[\mathrm{M}+\mathrm{H}]^{+}$: 485.2911. Found: 485.2931.

\section{Pharmacology}

Materials-Cell culture materials were purchased from Fisher SSI. Forskolin was purchased from Sigma-Aldrich. An expression plasmid containing a preprolactin leader sequence (PPLS) and an influenza hemagglutanin (HA) tag fused in frame upstream of the human GPR88 cDNA was prepared in a modified pcDNA3.1+mammalian expression vector by GenScript (Piscataway, NJ). Midi-prep DNA was prepared and the PPLS-HA-GPR88 construct was verified by sequencing.

Lance cAMP Assay Using Stable PPLS-HA-GPR88 CHO Cells-A stable PPLSHA-GPR88 CHO cell line was created by overexpressing the PPLS-HA-GPR88 construct in CHO cells. These cells were maintained in DMEM/F12 medium supplemented with 10\% fetal bovine serum (FBS), 100 units each of penicillin and streptomycin, and $400 \mu \mathrm{g} / \mathrm{mL}$ Geneticin (for antibiotic resistance). PerkinElmer's Lance Ultra kit (TRF0262) was used to detect cAMP accumulation. Stimulation buffer containing $1 \times$ Hank's balanced salt solution (HBSS), $5 \mathrm{mM}$ HEPES, 0.1\% BSA stabilizer, and $0.5 \mathrm{mM}$ final IBMX was prepared and titrated to 7.4 at room temperature. Serial dilutions of the test compounds $(5 \mu \mathrm{L})$ and 300 $\mathrm{nM}$ forskolin $(5 \mu \mathrm{L})$, both prepared at $4 \times$ the desired final concentration in $2 \% \mathrm{DMSO} /$ stimulation buffer, were added to a $96-$ well white $1 / 2$ area microplate (PerkinElmer). A cAMP standard curve was prepared at $4 \times$ the desired final concentration in stimulation buffer, and $5 \mu \mathrm{L}$ was added to the assay plate. Cells were lifted with versene and spun at $270 \mathrm{~g}$ for $10 \mathrm{~min}$. The cell pellet was resuspended in stimulation buffer and 4000 cells (10 $\mu \mathrm{L}$ ) were added to each well except wells containing the cAMP standard curve. After incubating for $30 \mathrm{~min}$ at RT, Eu-cAMP tracer and $\mathrm{u} L I G H T$-anti-cAMP working solutions 
were added per the manufacturer's instructions. After incubation at RT for $1 \mathrm{~h}$, the TRFRET signal (ex $337 \mathrm{~nm}$ ) was read on a CLARIOstar multimode plate reader (BMG Biotech, Cary NC).

Data Analysis-The TR-FRET signal $(665 \mathrm{~nm})$ was converted to fmol cAMP by interpolating from the standard cAMP curve. Fmol cAMP was plotted against the log of compound concentration and data were fit to a three-parameter logistic curve to generate $\mathrm{EC}_{50}$ values (Prism, version 6.0, GraphPad Software, Inc., San Diego, CA).

\section{Computational Studies}

Property Evaluation-Properties of fragments in the SAR substitution region were evaluated using semiempirical QM (MOPAC $7^{18}$ ) models at optimized geometries using this semiempirical Hamiltonian and with isotropic polarizabilities computed at B3LYP hybrid functional optimized geometries employing GAMESS-UK. ${ }^{22}$ Postcomputation analysis of the results was done with GRAPHA, a utility that extracts QM-energetic, electrostatic, hydrophobic, frontier-orbital energetic, Sterimol, shape, polar-non-polar surface area, volume, and thermodynamic descriptors. ${ }^{20}$ Substituted biphenyls were used in the computations, capping the substituent at the biphenyl end where it attaches to the central $\mathrm{N}$ in the ligands with a hydrogen.

Fragment/2D/3D QSAR-Properties compiled were analyzed to develop quantitative structure relationships in an effort to ascertain the key physiochemical properties modulating GPR88 $\ln \mathrm{EC}_{50}$ values as a function of substitution. Multivariate least-squares approaches embodied in the R PROJECT software for Statistical Computing (http://www.r-project.org).

Homology Modeling-Multiple and pairwise sequence alignments were made using the human sequence of GPR88 and sequences of known GPCR crystallographic template with their chimeric stabilizing proteins removed. Alignments were performed using Blosum scoring matrices employing CLUSTALX. An initial level homology model was developed using $\beta 1$-adrenergic receptor template (PDB: 5A8E) first employing Sali's MODELER $\mathrm{v} 9.11^{26}$ to obtain an initial backbone model through simulated annealing with topological constraints and employing the CHARMM potential function and then using SCWRL ${ }^{27}$ to predict the rotameric side chain states of nonconserved residues in the adrenergic receptor/ GPR88 alignment. AMBER12 was then used to energy minimized the structure using the Simmerling modified AMBER99 potential function and SANDER.

VINA/GLIDE Docking and MMGBSA Scoring- $(1 R, 2 R)$-2-PCCA was docked with both Autodock VINA ${ }^{29}$ and GLIDE-SP ${ }^{30}$ (Schrödinger) during an evaluation period. The Autodock VINA poses were MMGBSA rescored using AMBER12 GAFF+AMBER99SB potential functions and SANDER optimization, including $\mathrm{SQM}^{28}$ deduced electrostatic charges using scripted workflow developed at RTI for parallelized CPU/GPU implementation analogous to literature reports, ${ }^{31}$ while GLIDE-SP/XP-PrimeMMGBSA $^{30,32}$ results were used to deduce the lowest free energy poses using that methodology. The low-free energy poses of these two approaches were largely self- 
consistent and indicated a common mode of binding of $(1 R, 2 R)$-2-PCCA at the level of exploration of this early stage model.

\section{Supplementary Material}

Refer to Web version on PubMed Central for supplementary material.

\section{Acknowledgments}

We thank Tiffany Langston, Taylor Rosa, and Dr. Elaine Gay for their valuable technical assistance. We also thank Dr. Rangan Maitra for valuable discussions during the course of this study.

\section{Funding}

We are grateful to National Institute of Mental Health, National Institutes of Health, U.S. (Grant MH103708 to C.J.) for the financial support of this research.

\section{References}

1. Rask-Andersen M, Almen MS, Schioth HB. Trends in the exploitation of novel drug targets. Nat Rev Drug Discovery. 2011; 10:579-590. [PubMed: 21804595]

2. Armbruster BN, Roth BL. Mining the receptorome. J Biol Chem. 2005; 280:5129-5132. [PubMed: 15590622]

3. Civelli O, Reinscheid RK, Zhang Y, Wang Z, Fredriksson R, Schioth HB. G protein-coupled receptor deorphanizations. Annu Rev Pharmacol Toxicol. 2013; 53:127-146. [PubMed: 23020293]

4. (a) Mizushima K, Miyamoto Y, Tsukahara F, Hirai M, Sakaki Y, Ito T. A novel G-protein-coupled receptor gene expressed in striatum. Genomics. 2000; 69:314-321. [PubMed: 11056049] (b) Ghate A, Befort K, Becker JA, Filliol D, Bole-Feysot C, Demebele D, Jost B, Koch M, Kieffer BL. Identification of novel striatal genes by expression profiling in adult mouse brain. Neuroscience. 2007; 146:1182-1192. [PubMed: 17395390] (c) Massart R, Guilloux JP, Mignon V, Sokoloff P, Diaz J. Striatal GPR88 expression is confined to the whole projection neuron population and is regulated by dopaminergic and glutamatergic afferents. Eur J Neurosci. 2009; 30:397-414. [PubMed: 19656174] (d) Van Waes V, Tseng KY, Steiner H. GPR88-a putative signaling molecule predominantly expressed in the striatum: Cellular localization and developmental regulation. Basal Ganglia. 2011; 1:83-89. [PubMed: 21804954] (e) Becker JA, Befort K, Blad C, Filliol D, Ghate A, Dembele D, Thibault C, Koch M, Muller J, Lardenois A, Poch O, Kieffer BL. Transcriptome analysis identifies genes with enriched expression in the mouse central extended amygdala. Neuroscience. 2008; 156:950-965. [PubMed: 18786617]

5. Matsuoka T, Tsunoda M, Sumiyoshi T, Takasaki I, Tabuchi Y, Seo T, Tanaka K, Uehara T, Itoh H, Suzuki M, Kurachi M. Effect of MK-801 on gene expressions in the amygdala of rats. Synapse. 2008; 62:1-7. [PubMed: 17948890]

6. (a) Brandish PE, Su M, Holder DJ, Hodor P, Szumiloski J, Kleinhanz RR, Forbes JE, McWhorter ME, Duenwald SJ, Parrish ML, Na S, Liu Y, Phillips RL, Renger JJ, Sankaranarayanan S, Simon AJ, Scolnick EM. Regulation of gene expression by lithium and depletion of inositol in slices of adult rat cortex. Neuron. 2005; 45:861-872. [PubMed: 15797548] (b) Ogden CA, Rich ME, Schork NJ, Paulus MP, Geyer MA, Lohr JB, Kuczenski R, Niculescu AB. Candidate genes, pathways and mechanisms for bipolar (manic-depressive) and related disorders: an expanded convergent functional genomics approach. Mol Psychiatry. 2004; 9:1007-1029. [PubMed: 15314610]

7. Conti B, Maier R, Barr AM, Morale MC, Lu X, Sanna PP, Bilbe G, Hoyer D, Bartfai T. Regionspecific transcriptional changes following the three antidepressant treatments electro convulsive therapy, sleep deprivation and fluoxetine. Mol Psychiatry. 2007; 12:167-189. [PubMed: 17033635]

8. Befort K, Filliol D, Ghate A, Darcq E, Matifas A, Muller J, Lardenois A, Thibault C, Dembele D, Le Merrer J, Becker JA, Poch O, Kieffer BL. Mu-opioid receptor activation induces transcriptional plasticity in the central extended amygdala. Eur J Neurosci. 2008; 27:2973-2984. [PubMed: 18588537] 
9. Logue SF, Grauer SM, Paulsen J, Graf R, Taylor N, Sung MA, Zhang L, Hughes Z, Pulito VL, Liu F, Rosenzweig-Lipson S, Brandon NJ, Marquis KL, Bates B, Pausch M. The orphan GPCR, GPR88, modulates function of the striatal dopamine system: a possible therapeutic target for psychiatric disorders? Mol Cell Neurosci. 2009; 42:438-447. [PubMed: 19796684]

10. Quintana A, Sanz E, Wang W, Storey GP, Guler AD, Wanat MJ, Roller BA, La Torre A, Amieux PS, McKnight GS, Bamford NS, Palmiter RD. Lack of GPR88 enhances medium spiny neuron activity and alters motor- and cue-dependent behaviors. Nat Neurosci. 2012; 15:1547-1555. [PubMed: 23064379]

11. Meirsman AC, Le Merrer J, Pellissier LP, Diaz J, Clesse D, Kieffer BL, Becker JA. Mice lacking GPR88 show motor deficit, improved spatial learning, and low anxiety reversed by delta opioid antagonist. Biol Psychiatry. 2016; 79:917-927. [PubMed: 26188600]

12. Jin C, Decker AM, Huang XP, Gilmour BP, Blough BE, Roth BL, Hu Y, Gill JB, Zhang XP. Synthesis, pharmacological characterization, and structure-activity relationship studies of small molecular agonists for the orphan GPR88 receptor. ACS Chem Neurosci. 2014; 5:576-587. [PubMed: 24793972]

13. Bi Y, Dzierba CD, Fink C, Garcia Y, Green M, Han J, Kwon S, Kumi G, Liang Z, Liu Y, Qiao Y, Zhang Y, Zipp G, Burford N, Ferrante M, Bertekap R, Lewis M, Cacace A, Westphal RS, Kimball D, Bronson JJ, Macor JE. The discovery of potent agonists for GPR88, an orphan GPCR, for the potential treatment of CNS disorders. Bioorg Med Chem Lett. 2015; 25:1443-1447. [PubMed: 25754495]

14. Summerfield SG, Read K, Begley DJ, Obradovic T, Hidalgo IJ, Coggon S, Lewis AV, Porter RA, Jeffrey P. Central nervous system drug disposition: the relationship between in situ brain permeability and brain free fraction. J Pharmacol Exp Ther. 2007; 322:205-213. [PubMed: 17405866]

15. Ghose AK, Herbertz T, Hudkins RL, Dorsey BD, Mallamo JP. Knowledge-based, central nervous system (CNS) lead selection and lead optimization for CNS drug discovery. ACS Chem Neurosci. 2012; 3:50-68. [PubMed: 22267984]

16. Clark DE, Pickett SD. Computational methods for the prediction of 'drug-likeness'. Drug Discovery Today. 2000; 5:49-58.

17. Hitchcock SA, Pennington LD. Structure-brain exposure relationships. J Med Chem. 2006; 49:7559-7583. [PubMed: 17181137]

18. Stewart JJ. MOPAC: A semiempirical molecular orbital program. J Comput-Aided Mol Des. 1990; 4:1-105. [PubMed: 2197373]

19. (a) Verloop, A. The STERIMOL Approach to Drug Design. Marcel Dekker; New York: 1987. (b) Verloop, A.; Tipker, J. QSAR in Drug Design and Toxicology. Vol. 97. Elsevier; New York: 1987.

20. Harris D, Clayton T, Cook J, Sahbaie P, Halliwell RF, Furtmüller R, Huck S, Sieghart W, DeLorey TM. Selective influence on contextual memory: Physiochemical properties associated with selectivity of benzodiazepine ligands at GABAA receptors containing the a5 subunit. J Med Chem. 2008; 51:3788-3803. [PubMed: 18537233]

21. O’Boyle NM, Banck M, James CA, Morley C, Vandermeersch T, Hutchison GR. Open Babel: An open chemical toolbox. J Cheminf. 2011; 3:33.

22. Guest MF, Bush IJ, van Dam HJJ, Sherwood P, Thomas JMH, van Lenthe JH, Havenith RWA, Kendrick J. The GAMESS-UK electronic structure package: algorithms, developments and applications. Mol Phys. 2005; 103:719-747.

23. McCammon J, Lee C, Northrup S. Sidechain rotational isomerization in proteins: a mechanism involving gating and transient packing defects. J Am Chem Soc. 1983; 105:2232-2237.

24. Dawson MI, Harris DL, Liu G, Hobbs PD, Lange CW, Jong L, Bruey-Sedano N, James SY, Zhang XK, Peterson VJ, Leid M, Farhana L, Rishi AK, Fontana JA. Antagonist analogue of 6-[3'-(1adamantyl)-4' -hydroxyphenyl]-2-naphthalenecarboxylic acid (AHPN) family of apoptosis inducers that effectively blocks AHPN-induced apoptosis but not cell-cycle arrest. J Med Chem. 2004; 47:3518-3536. [PubMed: 15214780]

25. (a) Huang XP, Karpiak J, Kroeze WK, Zhu H, Chen X, Moy SS, Saddoris KA, Nikolova VD, Farrell MS, Wang S, Mangano TJ, Deshpande DA, Jiang A, Penn RB, Jin J, Koller BH, Kenakin T, Shoichet BK, Roth BL. Allosteric ligands for the pharmacologically dark receptors GPR68 and 
GPR65. Nature. 2015; 527:477-483. [PubMed: 26550826] (b) Heifetz A, Storer RI, McMurray G, James T, Morao I, Aldeghi M, Bodkin MJ, Biggin PC. Application of an integrated GPCR SARmodeling platform to explain the activation selectivity of human 5-HT2C over 5-HT2B. ACS Chem Biol. 2016; 11:1372-1382. [PubMed: 26900768]

26. Sali A, Blundell TL. Comparative protein modelling by satisfaction of spatial restraints. J Mol Biol. 1993; 234:779-815. [PubMed: 8254673]

27. Dunbrack RL Jr. Comparative modeling of CASP3 targets using PSI-BLAST and SCWRL. Proteins Suppl. 1999; 3:81-87.

28. Case, DA.; Darden, T.; Cheatham, TE., III; Simmerling, CL.; Wang, J.; Duke, RE.; Luo, R.; Walker, RC.; Zhang, W.; Merz, KM.; Roberts, B.; Hayik, S.; Roitberg, A.; Seabra, G.; Swails, J.; Götz, AW.; Kolossváry, I.; Wong, KF.; Paesani, F.; Vanicek, J.; Wolf, RM.; Liu, J.; Wu, X.; Brozell, SR.; Steinbrecher, T.; Gohlke, H.; Cai, Q.; Ye, X.; Wang, J.; Hsieh, M-J.; Cui, G.; Roe, DR.; Mathews, DH.; Seetin, MG.; Salomon-Ferrer, R.; Sagui, C.; Babin, V.; Luchko, T.; Gusarov, S.; Kovalenko, A.; Kollman, PA. AMBER. Vol. 12. University of California; San Francisco: 2012.

29. Trott O, Olson AJ. AutoDock Vina: improving the speed and accuracy of docking with a new scoring function, efficient optimization, and multithreading. J Comput Chem. 2010; 31:455-461. [PubMed: 19499576]

30. Friesner RA, Murphy RB, Repasky MP, Frye LL, Greenwood JR, Halgren TA, Sanschagrin PC, Mainz DT. Extra precision glide: docking and scoring incorporating a model of hydrophobic enclosure for protein-ligand complexes. J Med Chem. 2006; 49:6177-6196. [PubMed: 17034125]

31. Zhang X, Wong SE, Lightstone FC. Toward fully automated high performance computing drug discovery: a massively parallel virtual screening pipeline for docking and molecular mechanics/ generalized Born surface area rescoring to improve enrichment. J Chem Inf Model. 2014; 54:324337. [PubMed: 24358939]

32. Greenidge PA, Kramer C, Mozziconacci JC, Sherman W. Improving docking results via reranking of ensembles of ligand poses in multiple X-ray protein conformations with MM-GBSA. J Chem Inf Model. 2014; 54:2697-2717. [PubMed: 25266271] 

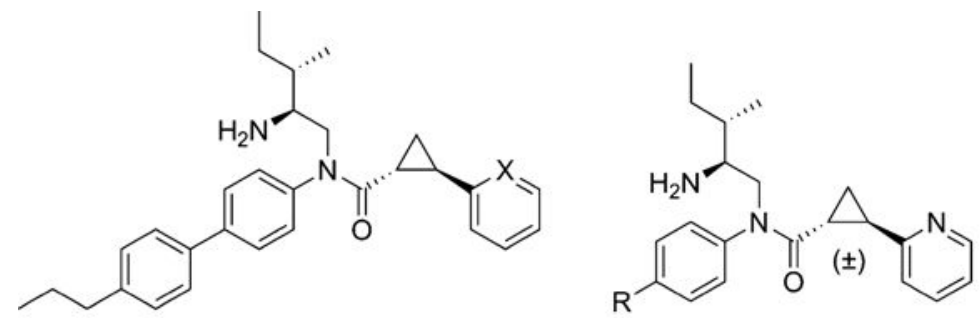

$$
\begin{aligned}
& 1 \mathrm{X}=\mathrm{N},( \pm) \text {-cyclopropyl, } \mathrm{EC}_{50}=116 \mathrm{nM} \\
& 2 \mathrm{X}=\mathrm{N},(R, R) \text {-cyclopropyl, } \mathrm{EC}_{50}=56 \mathrm{nM} \\
& 3 \mathrm{X}=\mathrm{C},(R, R) \text {-cyclopropyl, } \mathrm{EC}_{50}=233 \mathrm{nM}
\end{aligned}
$$

$$
\begin{aligned}
& \text { 4a R }=\mathrm{H}, \mathrm{EC}_{50}=2760 \mathrm{nM} \\
& \text { 4b R }=\text { hexyl, } \mathrm{EC}_{50}=421 \mathrm{nM} \\
& \text { 4c R }=\mathrm{Cy}, \mathrm{EC}_{50}=356 \mathrm{nM} \\
& \text { 4d R }=\mathrm{Ph}, \mathrm{EC}_{50}=283 \mathrm{nM} \\
& \text { 4e R }=\mathrm{PhO}, \mathrm{EC}_{50}=439 \mathrm{nM} \\
& \text { 4f } \mathrm{R}=\mathrm{Bn}, \mathrm{EC}_{50}=666 \mathrm{nM} \\
& \text { 4g R }=\mathrm{Ph}\left(\mathrm{CH}_{2}\right)_{2}, \mathrm{EC}_{50}=553 \mathrm{nM}
\end{aligned}
$$

Figure 1.

Structures of 2-PCCA (1), (1R,2R)-2-PCCA (2), and analogues $\mathbf{3}, \mathbf{4 a - g}$. 
<smiles>[R]c1ccc(-c2ccc(N(C[C@H](N)[C@@H](C)CC)C(=O)[C@@H]3C[C@H]3c3ccccn3)cc2)cc1</smiles>
5a $\mathrm{R}=4-\mathrm{Me}$
5j $\mathrm{R}=4-\mathrm{MeO}$
5s $\quad \mathrm{R}=4-\mathrm{NO}_{2}$
5b $\mathrm{R}=4-\mathrm{CF}_{3}$
5k $\mathrm{R}=4-\mathrm{CF}_{3} \mathrm{O}$
5t $\mathrm{R}=4-\mathrm{Ac}$
5c $\mathrm{R}=4$-Et
5I $\mathrm{R}=4$-EtO
5u $\mathrm{R}=4-\mathrm{CO}_{2} \mathrm{Et}$
5d $\mathrm{R}=4-i-\mathrm{Pr}$
$5 \mathrm{~m} \mathrm{R}=4-i-\mathrm{PrO}$
5v $\mathrm{R}=4-\mathrm{OH}$
5e $\mathrm{R}=4-i-\mathrm{Bu}$
5n $\mathrm{R}=3,4-\mathrm{diMeO}$
5w $\mathrm{R}=4-\mathrm{NH}_{2}$
5f $\mathrm{R}=4-t-\mathrm{Bu}$
5o $\mathrm{R}=3,4-\mathrm{MD}$
$5 x \quad \mathrm{R}=4-\mathrm{NMe}_{2}$
5g $R=4$-hexyl $5 p \quad R=4-F$
5y $\mathrm{R}=4-\mathrm{NHAC}$
$5 \mathrm{~h} \quad \mathrm{R}=4-\mathrm{Cy}$
5q $\mathrm{R}=4-\mathrm{Cl}$
$5 \mathbf{z}=4-\mathrm{CONH}_{2}$
5i $\mathrm{R}=4-\mathrm{Ph}$
5r $\mathrm{R}=4-\mathrm{CN}$
5aa $\mathrm{R}=4-\mathrm{CONMe}_{2}$

Figure 2.

Structure of 2-PCCA analogues 5a-aa. 

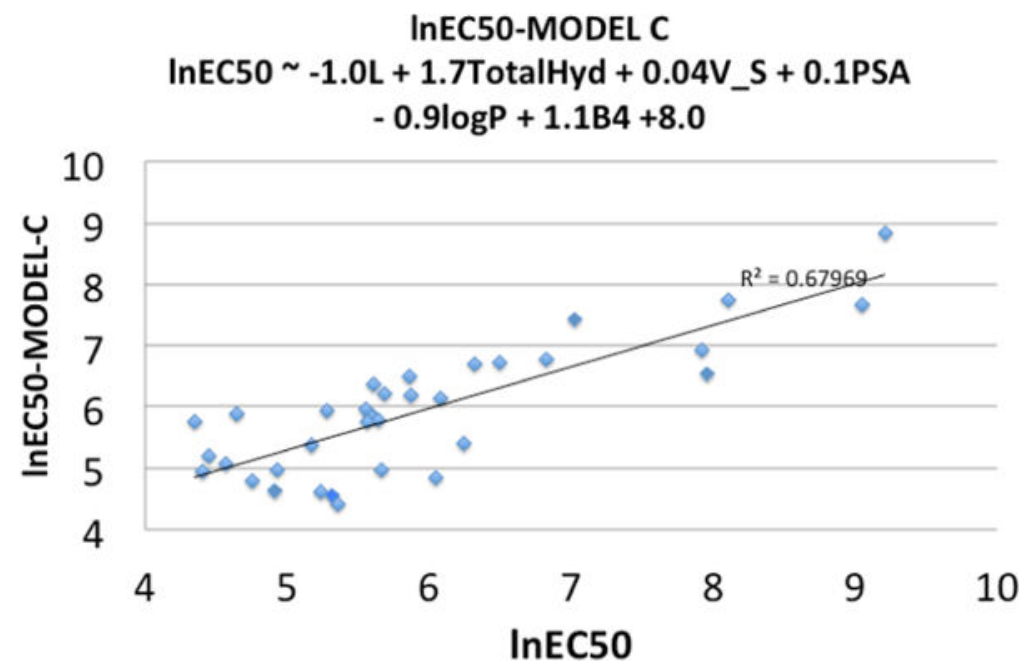

Figure 3.

Plot of experimental $\ln \mathrm{EC}_{50}$ vs model $\mathrm{C} \ln \mathrm{EC}_{50}$. 


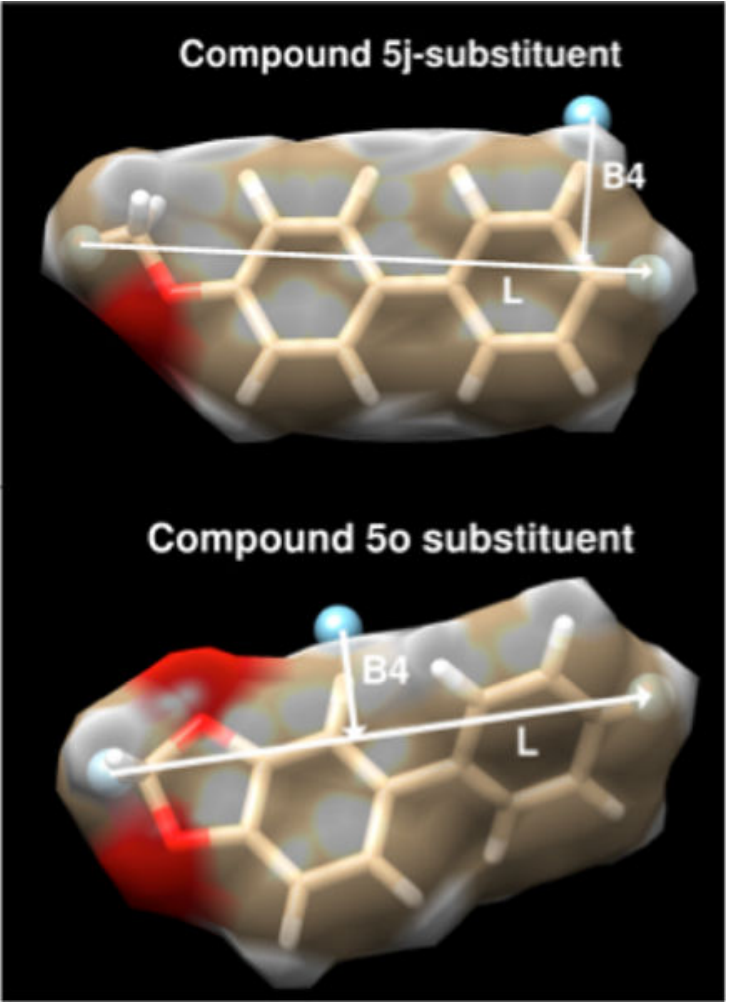

Figure 4.

Illustrations of the L- and B4-Sterimol metrics for $\mathbf{5 j}$ and $\mathbf{5 0}$. 
(A)

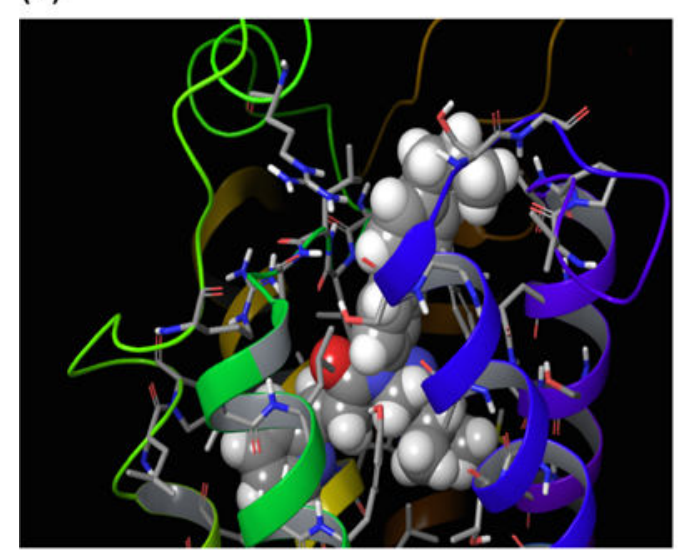

(B)

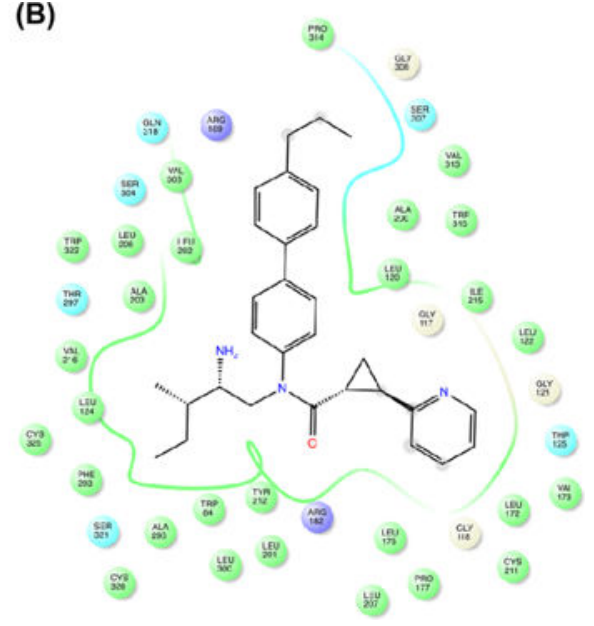

Figure 5.

Examination of an early stage homology model of GPR88 with $(1 R, 2 R)$-2-PCCA lowest MMGBSA scored pose in 3D (A) and LIGPLOT (B) format. Note the largely hydrophobic residues around the biphenyl scaffold portion indicated by the green line. 


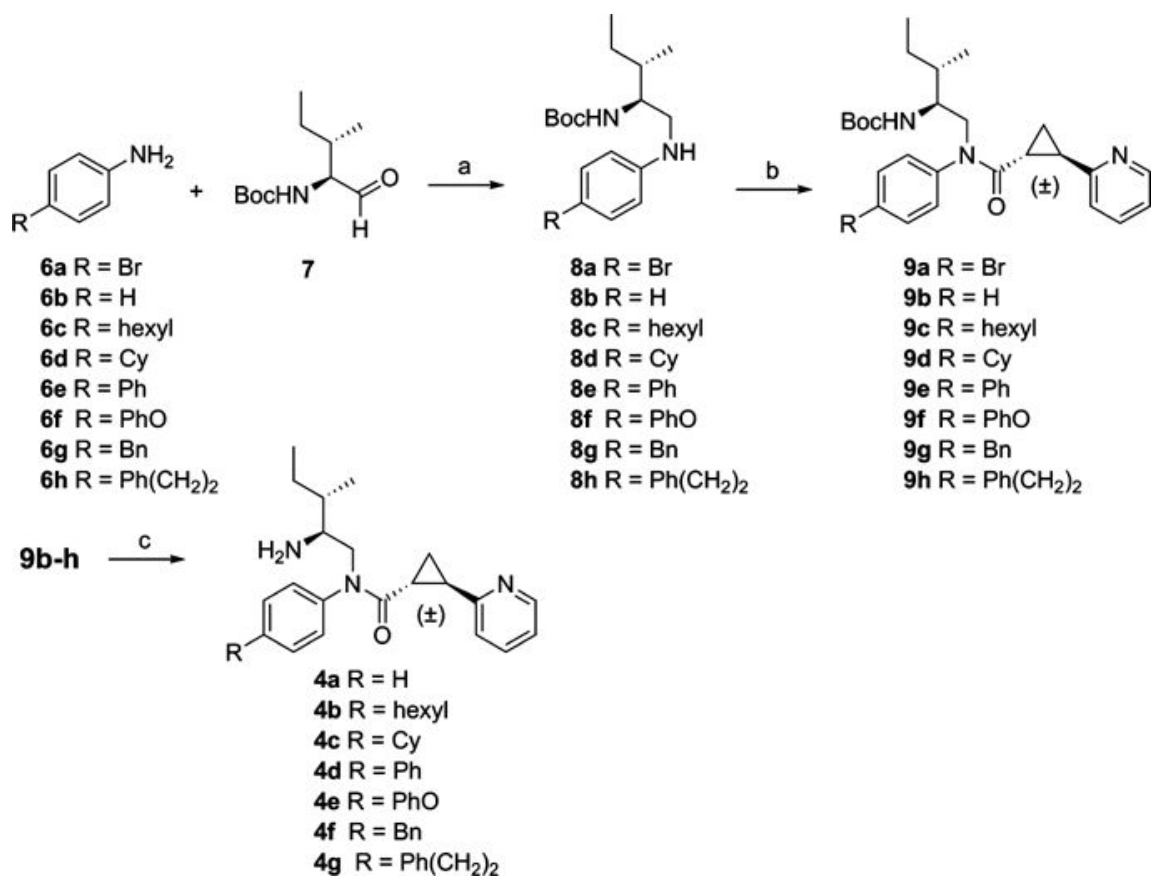

Scheme 1.

aReagents: (a) $\mathrm{NaBH}(\mathrm{OAc})_{3}, 1,2$-dichloroethane, rt, overnight; (b) ( \pm )-trans-2-(pyridin-2yl)cyclopropanecarboxylic acid/oxalyl chloride/DCM $/ 40{ }^{\circ} \mathrm{C} / 2 \mathrm{~h}$, concentrated, then $\mathbf{8 a}-\mathbf{h} /$ $\mathrm{Et}_{3} \mathrm{~N} / \mathrm{DCM}$, rt, overnight; (c) $4 \mathrm{M} \mathrm{HCl} /$ dioxane, DCM, rt, 6 h. 


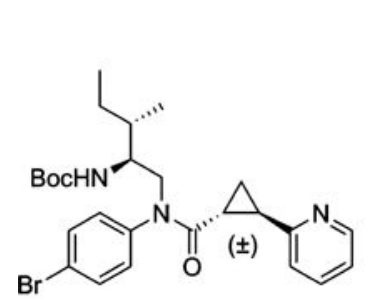

9a

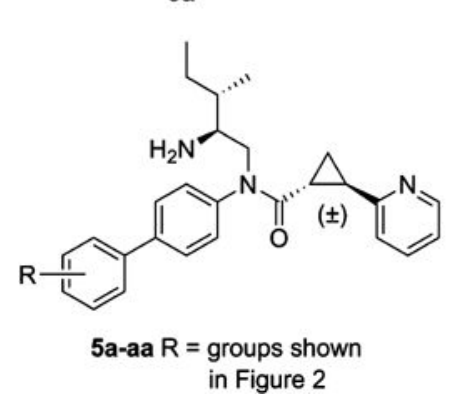

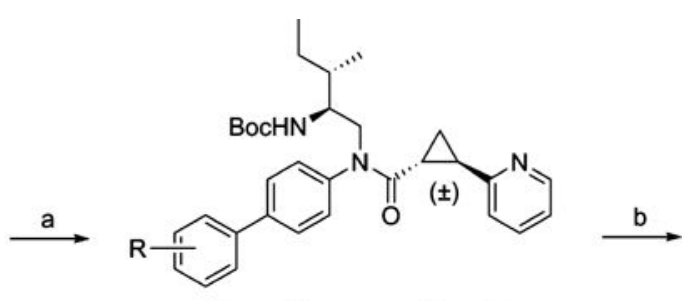

10a-aa $R=$ groups shown in Figure 2: 5a-aa

Scheme 2.

${ }^{a}$ Reagents: (a) arylboronic acid, $\mathrm{Pd}(\mathrm{dppf}) \mathrm{Cl}_{2} \cdot \mathrm{DCM}, \mathrm{K}_{3} \mathrm{PO}_{4}, \mathrm{DME} / \mathrm{H}_{2} \mathrm{O}$ (3:1), microwave, $160{ }^{\circ} \mathrm{C}, 6 \mathrm{~min}$; (b) $4 \mathrm{M} \mathrm{HCl} /$ dioxane, DCM, rt, 6 h. 

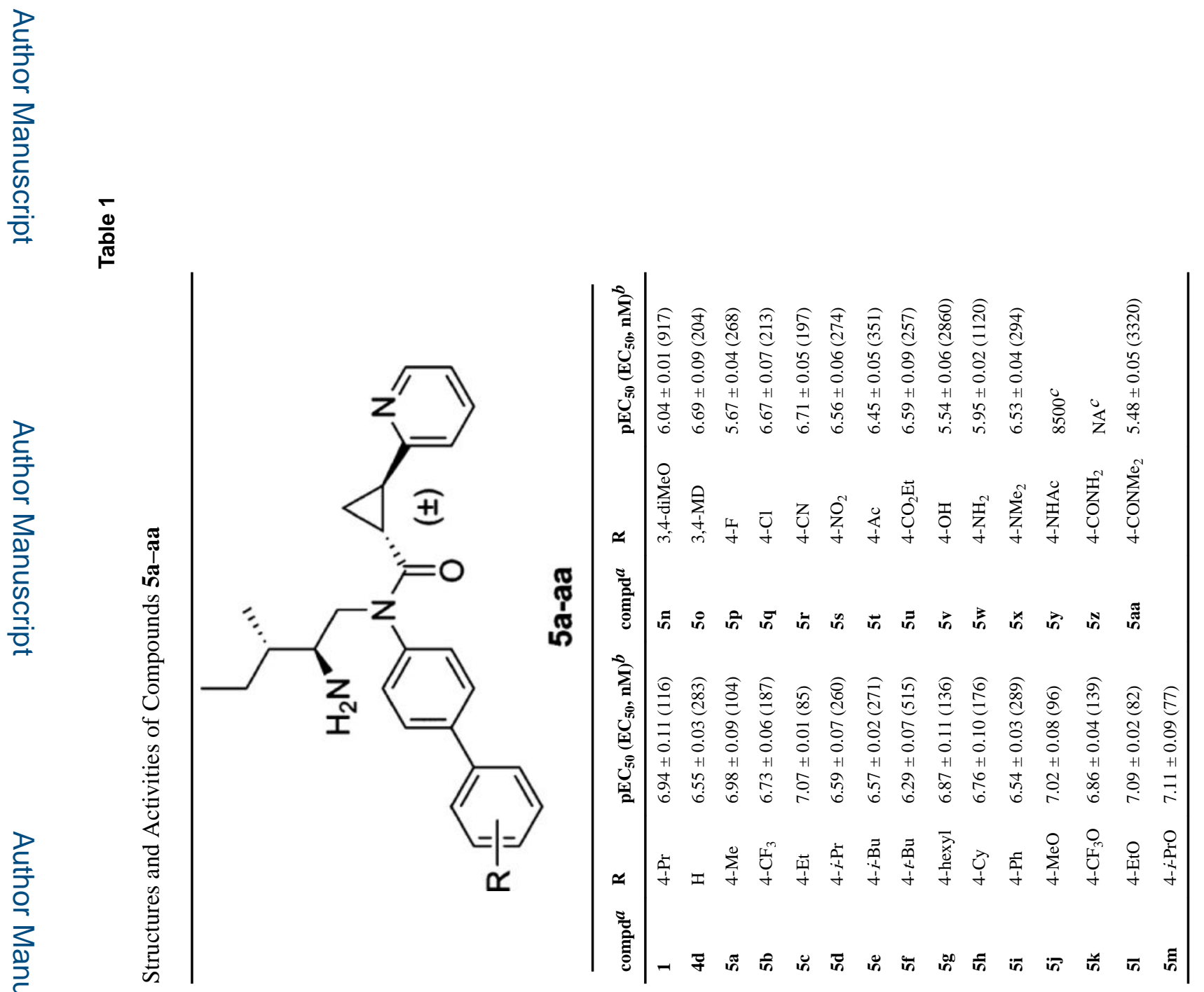

ACS Chem Neurosci. Author manuscript; available in PMC 2016 October 19. 

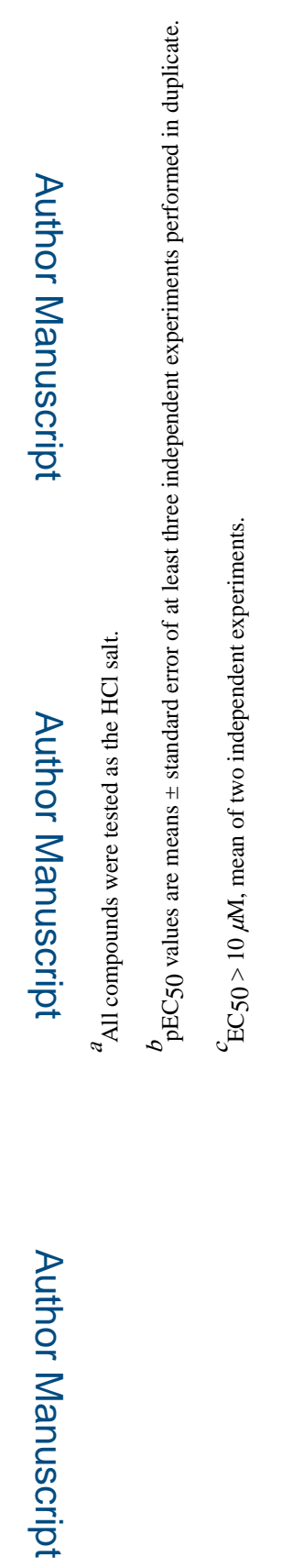

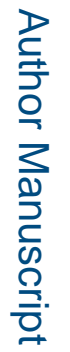

ACS Chem Neurosci. Author manuscript; available in PMC 2016 October 19. 


\section{Table 2}

Calculated Physiochemical Properties

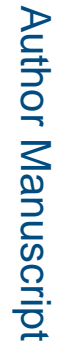

\begin{tabular}{ccccc}
\hline compd & $\mathbf{E C}_{\mathbf{5 0}}(\mathbf{n M})$ & $\operatorname{clog}^{a}$ & $\mathbf{T P S A}\left(\AA^{2}\right)^{\boldsymbol{a}}$ & $\operatorname{logBB}$ \\
\hline $\mathbf{1}$ & 116 & 6.19 & 59.22 & 0.20 \\
$\mathbf{5 a}$ & 104 & 5.30 & 59.22 & 0.07 \\
$\mathbf{5 c}$ & 85 & 5.74 & 59.22 & 0.14 \\
$\mathbf{5 j}$ & 96 & 4.63 & 68.45 & -0.17 \\
$\mathbf{5 l}$ & 82 & 4.99 & 68.45 & -0.12 \\
$\mathbf{5 m}$ & 77 & 5.40 & 68.45 & -0.05 \\
$\mathbf{5 0}$ & 204 & 4.41 & 77.68 & -0.34
\end{tabular}

${ }^{a} \operatorname{LogP}$ and TPSA were calculated using Instant JChem 5.4.0 (ChemAxon Ltd.). 


\section{Table 3}

QSAR Model Description ${ }^{a}$

\begin{tabular}{lcccc}
\hline \multicolumn{4}{c}{ model C: $\mathbf{l n} \mathbf{E C}_{\mathbf{5 0}} \sim \mathbf{L}+$ TotalHyd + V_S + PSA + $\mathbf{l o g P}+\mathbf{B 4}$} \\
\hline descriptor & Estimate coefficients & std error & $\mathbf{t}$-value & Pr $(>|t|)$ \\
\hline intercept & 8.05770 & 1.68999 & 4.768 & $5.23 \times 10^{-5}$ \\
L & -1.01903 & 0.46050 & -4.587 & $8.58 \times 10^{-5}$ \\
TotalHyd & 1.70887 & 0.46050 & 3.711 & 0.000907 \\
V_S & 0.04528 & 0.01001 & 4.523 & 0.000102 \\
PSA & 0.14556 & 0.02630 & 5.535 & $6.43 \times 10^{-6}$ \\
logP & -0.88637 & 0.37052 & -2.392 & 0.023701 \\
B4 & 1.11513 & 0.41208 & 2.706 & 0.011461
\end{tabular}

${ }^{a}$ Residual standard error $=0.7779$ on 28 degrees of freedom, multiple $R^{2}=0.6797$, adjusted $R^{2}=0.6111$, F-statistic $=9.903$ on 6 and 28 degrees of freedom, $p$-value $=7.063 \times 10^{-6}$. 